\title{
Geophysical Logs and Hydrologic Data for Eight Wells in the Coyote Spring Valley Area, Clark and Lincoln Counties, Nevada
}

By David L. Berger, Kathryn C. Kilroy, and Donald H. Schaefer

\section{U.S. GEOLOGICAL SURVEY}

Open-File Report 87-679

Prepared in cooperation with the STATE OF NEVADA, the

LAS VEGAS VALLEY WATER DISTRICT, and the

U. S. BUREAU OF RECLAMATION

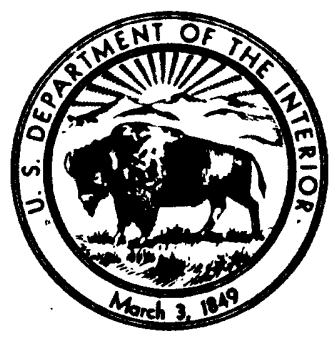

Carson City, Nevada 
DEPARTMENT OF THE INTERIOR

DONALD PAUL HODEL, Secretary

U.S. GEOLOGICAL SURVEY

Dallas L. Peck, Director

For additional information

Copies of this report may be write to:

purchased from:

U.S. Geological Survey

Room 227, Federal Building

705 North Plaza Street

Carson City, NV 89701
U.S. Geological Survey

Books and Open-File Reports Section Federal Center

Box 25425

Denver, CO 80225 


\section{CONTENTS}

Page

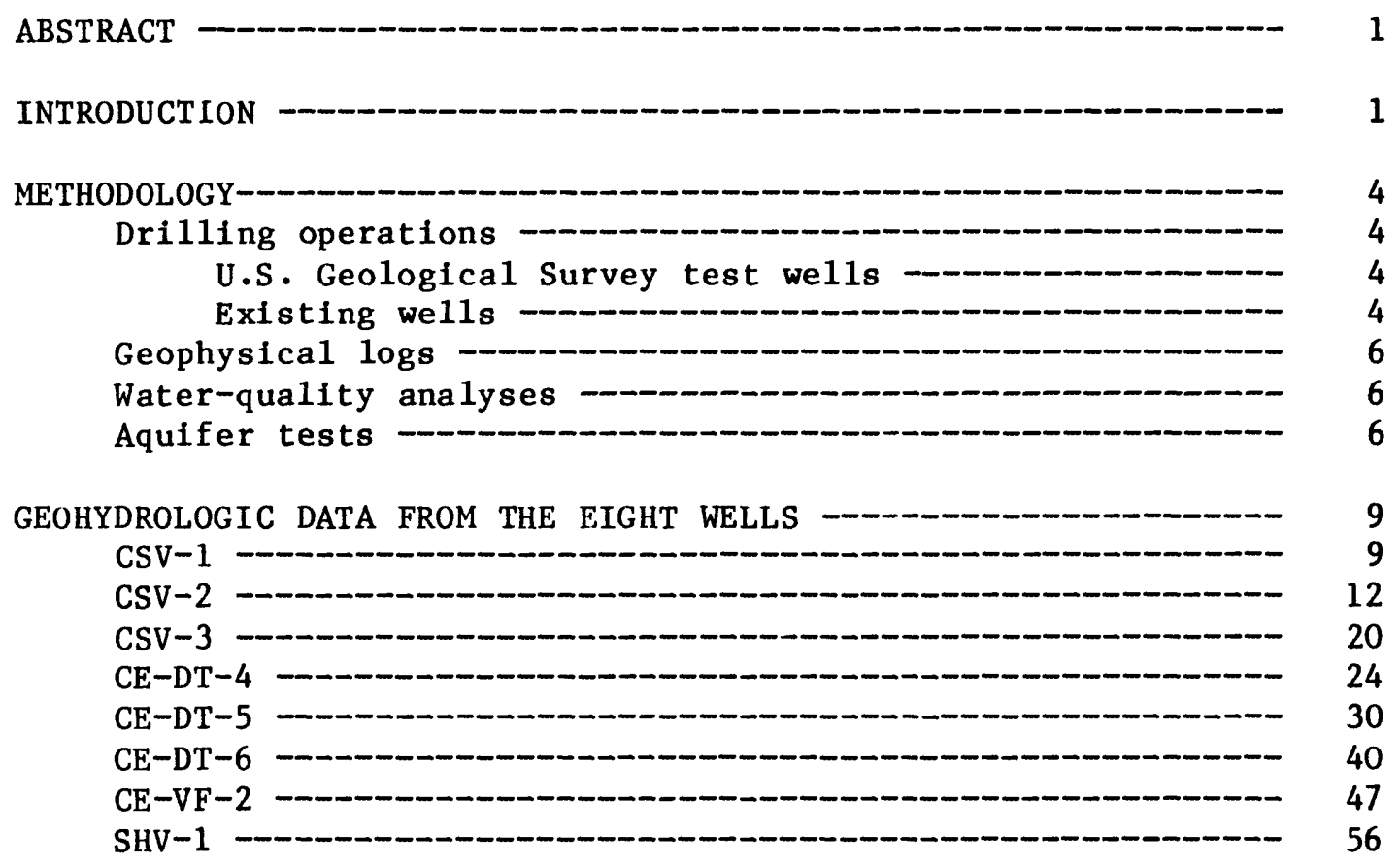

REFERENCES CITED - 59 
Figure 1. Map showing location of wells in study area

2-3. Graphs and logs showing data from Geological Survey test we11 CSV-1:

2. Drilling penetration rate and 1ithology 10

3. Natural gamma, neutron, gamma-gamma density, and water temperature

4-7. Graphs and logs showing data from Geological Survey test we11 CSV-2:

4. Driling penetration rate and lithology - 13

5. Natural gamma, neutron, gamma-gamma density, caliper, water temperature, and acoustic -

6. Drawdown versus time, June 7-8, 1986

7. Residual drawdown versus time, June 8, 1986 -_-_-_-_ 19

8-10. Graphs and logs showing data from Geological Survey test we11 CSV-3:

8. Drilling penetration rate and 1ithology - 21

9. Natural gamma, neutron, gamma-gamma density, caliper, and water temperature

10. Spontaneous potential, resistivity (long-normal and short-normal), and single-point resistance -

11-14. Graphs and logs showing data from MX test

we11 CE-DT-4:

11. Drilling penetration rate and 1ithology -25

12. Natura1 gamma, neutron, gamma-gamma density, caliper, and water temperature - -

13. Discharge versus time, December $20-23,1980------27$

14. Drawdown versus time, December 20-23, $1980-\ldots-$ 
Figures 15-17. Graphs and logs showing data from MX test we11 CE-DT-5:

15. Drilling penetration rate and 1ithology -

16. Water temperature - 32

17. Drawdown versus time, August 28 to September 27, 1981

18. Semilogarithmic plot of drawdown versus time at $\mathrm{MX}$ test we11 CE-DT-4, during pumping at $\mathrm{MX}$ test well CE-DT-5, August 28 to September 27, 1981

19-23. Graphs and logs showing data from MX test well CE-DT-6:

19. Drilling penetration rate and 11thology - 41

20. Water temperature and caliper 42

21. Data from continuous water-level recording transducer for October 28, 1985, to

January 28, 1986

22. Drawdown versus time, December 9-12, $1986-\cdots---44$

23. Residual drawdown versus time, December 12,1986

24-27. Graphs and logs showing data from $M X$ test we11 CE-VF-2:

24. Drilling penetration rate and 1ithology -

25. Natura1 gamma, neutron, gamma-gamma density, caliper, water temperature, and acoustic - 49

26. Drawdown versus time, February 6, $1986 \ldots 50$

27. Residual drawdown versus time, February 6, 1986

28. Logs showing natural gamma, neutron, gamma-gamma density, caliper, water temperature, and single-point resistance for test well SHV-1 
Table 1. Well locations, water levels, and other information

2. Summary of drilling and construction information for the eight wells

3. Summary of geophysical well logs 7

4. Water-quality data for selected we11s 8

5. Water levels during a 21.5-hour, constant-discharge aquifer test at U.S. Geological Survey test well CSV-2, June 7-8, 1986

6. Water levels during recovery from a 21.5-hour, constantdischarge aquifer test at U.S. Geological Survey test well CSV-2, June 8, 1986

7. Water levels during and after a 77-hour, constantdischarge aquifer test at MX test wel1 CE-DT-4, December 20-23, 1980

8. Water levels during a 30-day, constant-discharge aquifer test at $\mathrm{MX}$ test we11 CE-DT-5, August 28 to

September 28,1981

9. Water levels at observation test well CE-DT-4 during a 30-day, constant-discharge aquifer test at test well CE-DT-5, August 28 to September 27, 1981

10. Water levels during a 66-hour, constant-discharge aquifer test at MX test we11 CE-DT-6, December 9-12, 1986

11. Water levels during recovery from a 66-hour, constant-discharge aquifer test at MX test well CE-DT-6, December 12, 1986

12. Water levels during a 14-hour, constant-discharge aquifer test at MX test well CE-VF-2, February 6, 1986

13. Water levels during recovery from a 14-hour, constantdischarge aquifer test at $\mathrm{MX}$ test well $\mathrm{CE}-\mathrm{VF}-2$, February 6-7, 1986 


\section{CONVERSION FACTORS AND ABBREVIATIONS}

"Inch-pound" units of measure used in this report may be converted to metric (International System) units by using the following factors:

\begin{tabular}{|c|c|c|}
\hline Multiply & By & To obtain \\
\hline $\begin{array}{l}\text { Cubic foot per minute } \\
\left(\mathrm{ft}^{3} / \mathrm{min}\right)\end{array}$ & 0.02832 & $\begin{array}{l}\text { Cubic meter per minute } \\
\left(\mathrm{m}^{3} / \mathrm{min}\right)\end{array}$ \\
\hline Foot $(f t)$ & 0.3048 & Meter (m) \\
\hline Foot per minute $(\mathrm{ft} / \mathrm{min})$ & 0.3048 & Meter per minute $(\mathrm{m} / \mathrm{min})$ \\
\hline Gallon (gal) & 3.785 & Liter (L) \\
\hline Gallon per day (gal/day) & 3.785 & Liter per day (L/day) \\
\hline Gallon per minute (gal/min) & 0.06309 & Liter per second $(L / s)$ \\
\hline Inch (in.) & 25.40 & Millimeter (mm) \\
\hline $\begin{array}{l}\text { Mile (mi) } \\
\text { Square mile }\left(\mathrm{mi}^{2}\right)\end{array}$ & $\begin{array}{l}1.609 \\
2.590\end{array}$ & $\begin{array}{l}\text { Kilometer }(\mathrm{km}) \\
\text { Square kilometer }\left(\mathrm{km}^{2}\right)\end{array}$ \\
\hline
\end{tabular}

For temperature, degrees Celsius $\left({ }^{\circ} \mathrm{C}\right)$ may be converted to degrees Fahrenheit $\left({ }^{\circ} \mathrm{F}\right)$ by using the formula ${ }^{\circ} \mathrm{F}=\left[(1.8)\left({ }^{\circ} \mathrm{C}\right)\right]+32$.

\section{ALTITUDE DATUM}

In this report, "sea leve1" refers to the National Geodetic Vertical Datum of 1929 (NGVD of 1929), which is derived from a general adjustment of the first-order leveling networks of both the United States and Canada. 


\title{
GEOPHYSICAL LOGS AND HYDROLOGIC DATA FOR EIGHT WELLS \\ IN THE COYOTE SPRING VALLEY AREA, \\ CLARK AND LINCOLN COUNTIES, NEVADA
}

By David L. Berger, Kathryn C. Kilroy, and Donald H. Schaefer

\begin{abstract}
Geophysica1 logs, drilling operations, pump-test data, and waterquality determinations are presented for eight wells in the Coyote Spring Valley area of southeastern Nevada. The wells are in an area where thick units of Paleozolc carbonate rock are overlain by Tertiary semiconsolidated basin-fill deposits and Quaternary alluvial deposits. Data collected by the U.S. Geological Survey were augmented with data from previous investigations; however, complete sets of logs and other data are not available for all eight wells. Geophysical data presented include natural-gamma, neutron, gamma-gamma density, caliper, watertemperature, acoustic, single-point resistance, long- and short-normal resistivity, and spontaneous-potential logs. Drilling penetration rates, lithologic columns, and well construction are also summarized and presented. Measurements of drawdown and recovery during and after constant-discharge pumping periods are also included. Also presented are results of chemical and physical analyses for major-ion chemistry, trace constituents, stable and radioactive isotopes, temperature, $\mathrm{pH}$, specific conductance, and dissolved oxygen.
\end{abstract}

\section{INTRODUCTION}

In 1985, the U.S. Geological Survey began investigating the geology and hydrology in southern Nevada to better understand the regional carbonate-rock aquifer systems. This study, known as the Nevada Carbonate Aquifers Program (NCAP), is being made in cooperation with the State of Nevada, the Las Vegas Valley Water District, and the U.S. Bureau of Reclamation.

As part of the NCAP, geophysical, lithologic, hydrologic, and geochemical data were collected from eight wells: three wells drilled by the Geological Survey in 1985, four drilled by the U.S. Air Force for the Nevada-Utah MX missile-siting investigation, and one well that apparently was drilled as a stock well prior to 1958 . Figure 1 shows the location of all eight we1ls, and table 1 lists identifying information for each well. 


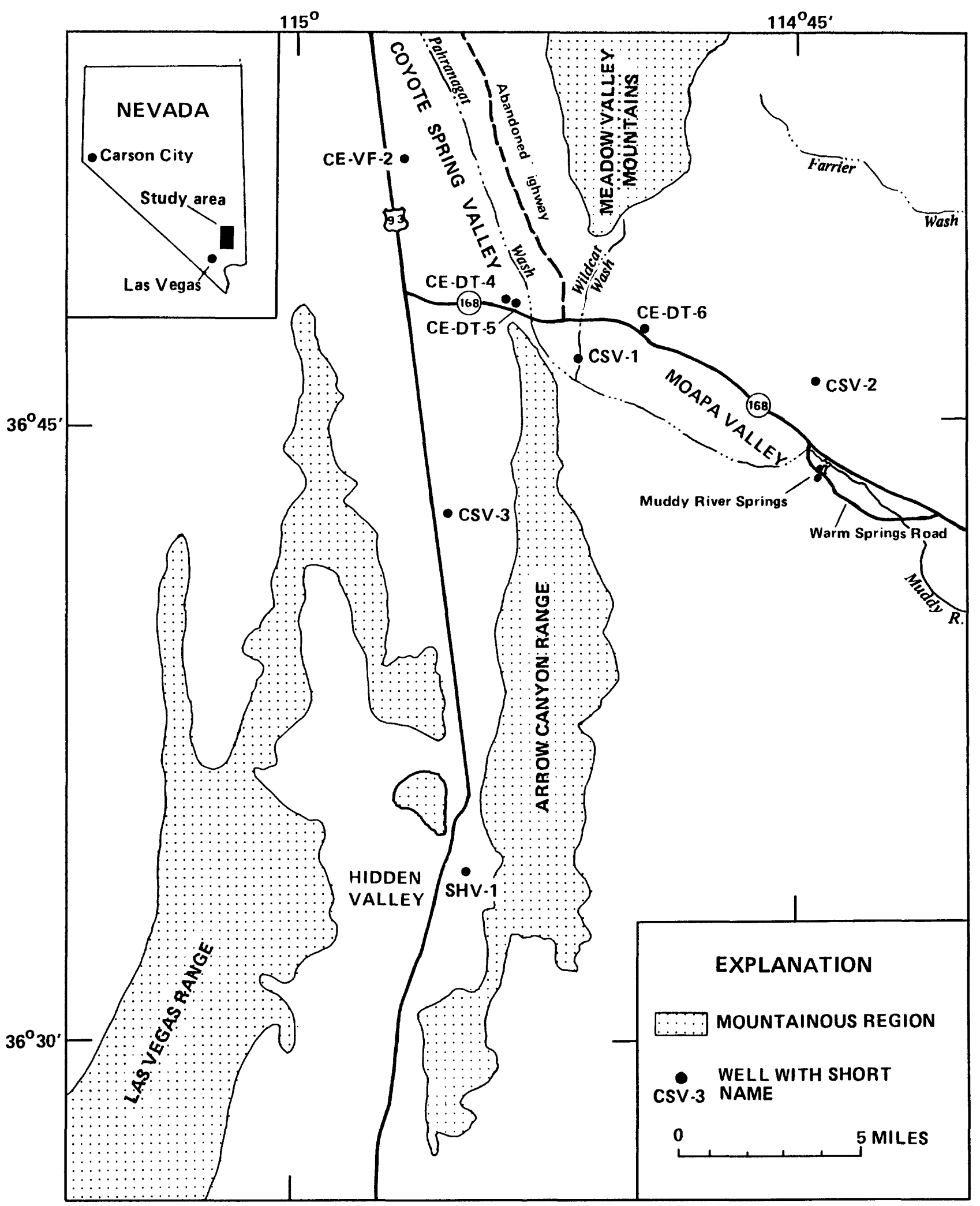

FIGURE 1.--Location of wells in study area. 
TABLE 1.--Well locations, water levels, and other information for the eight wells and for cne additional observation well in the study area

\begin{tabular}{|c|c|c|c|c|c|c|c|c|c|c|c|c|c|c|c|}
\hline \multirow{3}{*}{$\begin{array}{l}\begin{array}{l}\text { Short } \\
\text { wel1 } \\
\text { name } 1\end{array} \\
\operatorname{csv}-1\end{array}$} & \multirow{2}{*}{\multicolumn{4}{|c|}{$\begin{array}{c}\text { Local stte } \\
\text { Ident If Ication } 1\end{array}$}} & \multirow{2}{*}{\multicolumn{5}{|c|}{$\begin{array}{l}\text { Let tiude and longltude } \\
\text { (degrees, minutes, } \\
\text { seconds) }\end{array}$}} & \multirow{3}{*}{$\begin{array}{c}\begin{array}{c}\text { Date of } \\
\text { construc- } \\
\text { tfun }\end{array} \\
10-16-85\end{array}$} & \multirow{3}{*}{$\begin{array}{c}\begin{array}{c}\text { Land- } \\
\text { nurf nce } \\
\text { alt tude } \\
\text { (feet above } \\
\text { sea level) }\end{array} \\
2,158.6\end{array}$} & \multirow{3}{*}{$\begin{array}{c}\begin{array}{c}\text { Well depth } \\
\text { (fect } \\
\text { below } \\
\text { land } \\
\text { ourface) }\end{array} \\
765\end{array}$} & \multicolumn{3}{|c|}{ Water level } \\
\hline & & & & & & & & & & & & & Date & $\begin{array}{l}\text { Feet below } \\
\text { land burface }\end{array}$ & $\begin{array}{l}\text { Source of } \\
\text { measurement }\end{array}$ \\
\hline & 219 & 513 & E64 & 31DAAD1 & 36 & 46 & 01 & 114 & 5143 & & & & $\begin{array}{l}11-11-85 \\
12-17-85 \\
09-11-87\end{array}$ & $\begin{array}{l}343.90 \\
344.70 \\
343.44\end{array}$ & $\begin{array}{l}\text { USGS } \\
\text { USGS } \\
\text { USGS }\end{array}$ \\
\hline $\operatorname{csv}-2$ & 219 & $\$ 13$ & $E 65$ & $28 B D A C l$ & 36 & 46 & 50 & 114 & 4320 & $10-26-85$ & $2,185.9$ & 478 & $\begin{array}{l}10-27-85 \\
12-30-85 \\
06-07-86 \\
09-11-87\end{array}$ & $\begin{array}{l}391.80 \\
390.21 \\
390.76 \\
390.94\end{array}$ & $\begin{array}{l}\text { USGS } \\
\text { USGS } \\
\text { USGS } \\
\text { USGS }\end{array}$ \\
\hline $\operatorname{csv}-3$ & 210 & 514 & F.63 & $28 \mathrm{ACDCl}$ & 36 & 41 & 27 & 114 & S5 30 & $11-24-85$ & $2,414.3$ & 780 & $\begin{array}{l}12-20-85 \\
02-19-86 \\
09-13-87\end{array}$ & $\begin{array}{l}585.00 \\
587.50 \\
589.45\end{array}$ & $\begin{array}{l}\text { UsGs } \\
\text { USGS } \\
\text { usGS }\end{array}$ \\
\hline$C E-D T-4$ & 210 & s13 & E63 & 23DDDC1 & 36 & 47 & 43 & 114 & 5331 & $11-20-80$ & $2,172.6$ & 669 & $\begin{array}{l}12-12-80 \\
06-28-81 \\
03-14-85 \\
12-04-85 \\
09-11-87 \\
09-13-87\end{array}$ & $\begin{array}{l}354.00 \\
352.30 \\
351.77 \\
350.00 \\
351.79 \\
351.75\end{array}$ & $\begin{array}{l}\text { ERTEC } \\
\text { ERTEC } \\
\text { USGS } \\
\text { USGS } \\
\text { USGS } \\
\text { USGS }\end{array}$ \\
\hline$C E-D T-5$ & 210 & 513 & E63 & 230DDD1 & 36 & 47 & 41 & 114 & 5328 & $04-14-81$ & $2,169.1$ & 628 & $\begin{array}{l}05-06-81 \\
07-04-81 \\
08-13-81 \\
03-14-85 \\
12-03-85 \\
09-11-87 \\
09-13-87\end{array}$ & $\begin{array}{l}352.40 \\
348.50 \\
349.20 \\
347.84 \\
348.50 \\
348.72 \\
348.86\end{array}$ & $\begin{array}{l}\text { ERTEC } \\
\text { ERTEC } \\
\text { ERTEC } \\
\text { USGS } \\
\text { USGS } \\
\text { USGS } \\
\text { USGS }\end{array}$ \\
\hline CE-DT-6 & 210 & S13 & E64 & 35ACAAI & 36 & 46 & 04 & 114 & $47 \quad 13$ & $05-21-81$ & $2,274.6$ & 937 & $\begin{array}{l}06-03-81 \\
06-06-81 \\
07-11-81 \\
08-11-81 \\
03-14-85 \\
06-18-85 \\
09-11-87 \\
09-13-87\end{array}$ & $\begin{array}{l}458.00 \\
457.00 \\
457.40 \\
457.80 \\
457.37 \\
457.34 \\
459.16 \\
459.47\end{array}$ & $\begin{array}{l}\text { ERTEC } \\
\text { ERTEC } \\
\text { ERTEC } \\
\text { ERTEC } \\
\text { USGS } \\
\text { USGS } \\
\text { USGS } \\
\text { USGS }\end{array}$ \\
\hline CE-YF-1 & 210 & $\mathbf{s 1 2}$ & $\mathbf{E 6 3}$ & $290 D C C 1$ & 36 & 52 & 32 & 114 & $55 \quad 44$ & $11-13-80$ & $2,464.2$ & 714 & $\begin{array}{l}01-28-86 \\
01-29-86 \\
02-05-86 \\
02-06-86 \\
09-13-87\end{array}$ & $\begin{array}{l}542.98 \\
543.00 \\
542.80 \\
542.70 \\
548.55\end{array}$ & $\begin{array}{l}\text { USGS } \\
\text { USGS } \\
\text { USGS } \\
\text { USGS } \\
\text { USGS }\end{array}$ \\
\hline \multirow[t]{2}{*}{$C E-V_{F}-2$} & 210 & 512 & E63 & 29DABC1 & 36 & 52 & 27 & 114 & $55 \quad 44$ & $12-15-80$ & $2,466.9$ & 1,221 & $\begin{array}{l}07-11-81 \\
09-29-81\end{array}$ & $\begin{array}{l}611.70 \\
609.00\end{array}$ & $\begin{array}{l}\text { ERTEC } \\
\text { FRTEC }\end{array}$ \\
\hline & & & & & & & & & & & & & $\begin{array}{l}02-05-85 \\
11-25-85 \\
01-28-86 \\
01-29-86 \\
02-04-86\end{array}$ & $\begin{array}{l}603.10 \\
602.00 \\
604.62 \\
604.10 \\
604.30\end{array}$ & $\begin{array}{l}\text { USGS } \\
\text { USGS } \\
\text { USGS } \\
\text { USGS } \\
\text { USGS }\end{array}$ \\
\hline SHV-1 & 217 & S16 & $\mathbf{E} 63$ & O9DDABI & 36 & 33 & $O B$ & 114 & 5530 & unknown & $2,648.8$ & 920 & $\begin{array}{l}12-30-85 \\
09-13-87\end{array}$ & $\begin{array}{l}833.20 \\
831.00\end{array}$ & $\begin{array}{l}\text { USGS } \\
\text { USGS }\end{array}$ \\
\hline
\end{tabular}

1 In tils table, each site is Ident ffled by a short well name, the local (Nevada) site-1dentification system, and by lat tude and longltude. Except for this table, only the short well name (for example, shv-1) is used in the report, for conventence. The local ste-1dentiflcation system is based on an index of hydrographic areas in Nevada (Rush, ig68) and on the rectangular subdivision of the publlc lands referenced to the Mount Diablo base line and meridian. Bach number consista of four units sepnrated by spaces: The first unit is the hydrographic area number. The second unit ts the township, preceded by an $N$ or $S$ to Indlcate location north or south of the base 11 ne. The third unt is the range, preceded by an $E$ to indicate location east of the meridian. The fourth unlt consigtg of the oection number and letters designating the quarter section, quarter-quarter section, and $B O$ on ( $A, B, C$, and $D$ Indlcate the northeast, northwest, southwest, and southeast quarters, respectively), followed by a number Indicating the sequence in which the well was recorded. For example, well 217 S16 E63 O9DDABI is in Hidden Valley (hydrographic area 217). It is the $f$ irst well recorded in the NWt of the NEt of the SEk of the SEt of section 9, Townghip 16 South, Range 63 East, Mount Diablo base llne and merldian.

2 ERTEC, Ertec Western, Inc.; USGS, U.S. Geologlcal Survey. 
The wells are in an area encompassing approximately $400 \mathrm{mi}^{2}$; it includes Coyote Spring Valley, Hidden Valley, and the Muddy River Springs area. This area lies within the southern portion of the Basin and Range Province. Tertiary basin-fill deposits and Quaternary alluvial deposits overlie thick units of Paleozoic carbonate rock. Five wells in this study area are completed in the carbonate-rock aquifer.

The data were collected for future analysis to determine in-situ hydrogeologic conditions of the carbonate-rock aquifer, and thereby increase the understanding of regional flow systems in southern Nevada.

The authors thank the residents of nearby Moapa Valley for their assistance in completing the field work. A special thanks is given to Robert Plummer for his hospitality. We also acknowledge the support and cooperation of the U.S. Air Force for the use of their data from the exploratory MX project.

METHODOLOGY

Drilling Operations

\section{U.S. Geological Survey Test Wells}

The Geological Survey drilled three test we11s: CSV-1, CSV-2, and CSV-3 during October, November, and December 1985. The drilling was done by hydraulic-rotary and air-foam methods. Table 2 1ists data on drill bit sizes, casing, and drilling fluid used at these sites. Drill cuttings were collected at 10-foot intervals and where 1ithologic changes occurred. A solution containing a lithium bromide concentration of $20 \mathrm{milligrams}$ per 1iter $(\mathrm{mg} / \mathrm{L})$ constituted the water part of the driling fluid at each site. When chemical samples were later collected, a sufficient volume of water was first pumped from the aquifer so that the bromide tracer was reduced to low, natural concentrations. At each site, the surface casing was sealed and enclosed with a 5-foot section of 12-inch-diameter galvanized pipe, which was then cemented in place and capped with steel flat stock and locked.

\section{Existing We11s}

During 1980 and 1981, Ertec Western, Inc. (formerly Fugro National, Inc.) made hydrologic investigations in the Coyote Spring Valley area for the U.S. Air Force. Four deep exploratory wells were drilled and analyzed by geophysical logs, aquifer tests, and 1ithologic identification to assess ground-water potential as part of MX missile siting investigations. Ertec Western, Inc. (1981) has published data for two of these wells (CE-DT-4 and CE-DT-5). Some of the information presented in this report is from that published reference, while the remainder is from field notes and other documents provided by the U.S. Air Force and Ertec. A brief summary on location and drilling operations of the 
four MX wells is presented in the section on geohydrologic data in this report. Table 2 lists the bit sizes, casing information, and type of drilling fluid used at the MX sites. During drilling, lithologic samples were collected at 5-foot intervals, at apparent formation changes, and wherever changes were observed in drilling conditions. Upon completion of drilling, spontaneous-potential, resistivity, natural gamma, 3-d velocity, and 3-diameter-caliper logs were run in the wells. In addition, video logs were run in CE-DT-4, CE-DT-5 and CE-DT-6. An abandoned stock well (SHV-1) was also investigated and the data are presented in the section on geohydrologic data in this report.

TABLE 2.--Sumnary of drilling and construction information for the eight wells

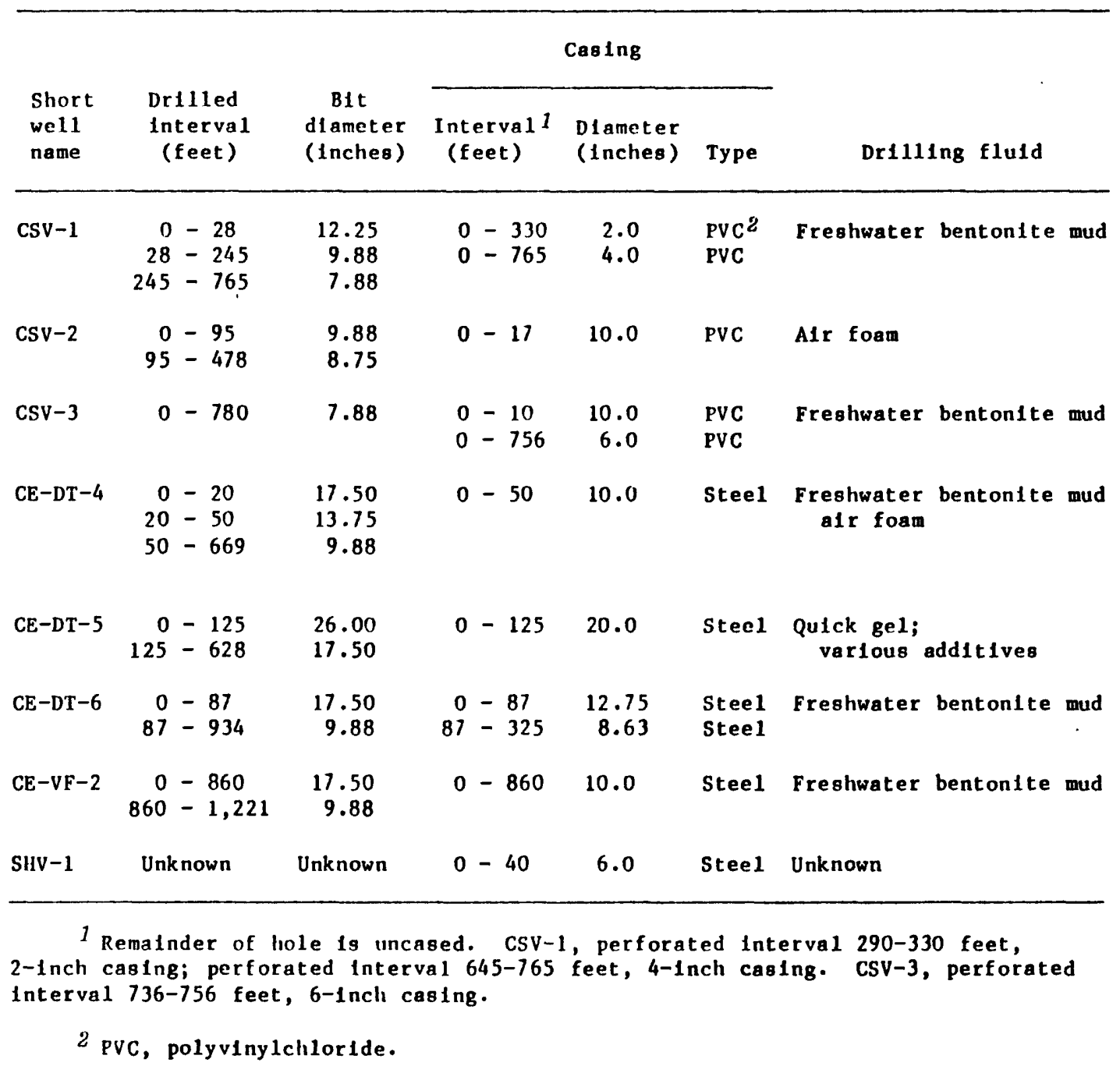


The geophysical we1l-log data collected by the Geological Survey were recorded simultaneously on an analog strip chart and a digital magnetic cassette. All well logs are referenced with zero depth at land surface. Table 3 lists the types of logs and depth intervals for each test we1l. Graphs of the logs are presented in the section on geohydrologic data in this report. Calibrations for the neutron and gamma-gamma tools are available at the Geological Survey Carson City office. The MX geophysical logs may be obtained from either the Nevada State Engineer or the U.S. Geological Survey, Carson City, Nev.

\section{Water-Quality Analyses}

Water-quality samples were collected by the Geological Survey during 1986 at wells CSV-2, CE-DT-6, CE-VF-2, and SHV-1. Previous water-quality samples were collected by the Geological Survey in 1980 and 1981 at CE-DT-4 and CE-DT-5. All samples were analyzed at the U.S. Geological Survey Central Laboratory in Denver, Colo. In addition, isotope analyses were performed at the Central Laboratory for carbon-13, carbon-14, and tritium; at the U.S. Geological Survey. Research Laboratory in Reston, Va., for deuterium and oxygen-18; and at the Global Geochemistry Corporation Laboratory in Canoga Park, Calif., for sulfur-34. Results of the above analyses are presented in table 4. The data include major-ion chemistry, trace constituents, stable and radioactive isotopes, temperature, $\mathrm{pH}$, specific conductance, and dissolved oxygen.

We11s CSV-2 and CE-VF-2 were pumped a total of 130,000 and 64,000 gallons, respectively, before sampling. Well SHV-1 was bailed three well volumes before sampling; however, the bromide concentration of the (ground water) sample was still above background concentrations of the area, making this sample questionable. We11 CE-DT-6 is used as a water-supply well for the Moapa Valley Water District and, therefore, was pumping for several months before it was sampled. We11s CE-DT-4 and CE-DT-5 were drilled and tested as part of the MX project, so both wells were pumping for several days before they were sampled. Samples from all the MX wells are good representatives of water in the aquifer.

\section{Aquifer Tests}

Water-leve1 drawdowns and recoveries were observed during aquifer tests on five wells that penetrate carbonate bedrock. These tests were carried out between 1980 and 1986 by Hydro Search, Inc.; Ertec Western, Inc.; and the Geological Survey. Results of these tests are presented in tables 5-13 and in figures $6,7,13,14,17,18,22,23,26$, and 27 in the section on geohydrologic data in this report.

Table 1 includes water-level data for the eight wells studied. Currently (1987), the Geological Survey is operating a continuous water-leve1 recorder in wel1 CE-DT-4. Water-level information obtained during aquifer tests is also reported in the section on geohydrologic data in this report. 
TABLE 3.--Summary of geophysical well loge presented in this report

\begin{tabular}{|c|c|c|c|c|c|c|c|}
\hline $\begin{array}{l}\text { Short } \\
\text { we } 11 \\
\text { name }\end{array}$ & $\begin{array}{l}\text { Date } \\
\text { logged }\end{array}$ & & Source 1 & Sulte of $\log s$ performed & $\begin{array}{r}\text { Log } \\
\text { Inte } \\
\text { (fe }\end{array}$ & $\begin{array}{l}\text { gged } \\
\text { erval } \\
\text { eet) }\end{array}$ & $\begin{array}{r}\text { Location } \\
\text { of loge } \\
\text { (figure } \\
\text { number) }\end{array}$ \\
\hline $\operatorname{csv}-1$ & $11-20-85$ & USGS, & Denver & $\begin{array}{l}\text { Natural gamma } \\
\text { Neutron } \\
\text { Gamma-gamma density } \\
\text { Water temperature }\end{array}$ & $\begin{array}{r}0 \\
295 \\
250 \\
344\end{array}$ & $\begin{array}{l}-750 \\
-750 \\
-750 \\
-750\end{array}$ & $\begin{array}{l}3 \\
3 \\
3 \\
3\end{array}$ \\
\hline CSV-2 & $11-19-85$ & USGS, & Denver & $\begin{array}{l}\text { Natural gamma } \\
\text { Neutron } \\
\text { Gamma-gamma density } \\
\text { Caliper } \\
\text { Water temperature } \\
\text { Acoustic }\end{array}$ & $\begin{array}{r}0 \\
0 \\
0 \\
0 \\
392 \\
392\end{array}$ & $\begin{array}{l}-466 \\
-466 \\
-466 \\
-466 \\
-478 \\
-466\end{array}$ & $\begin{array}{l}5 \\
5 \\
5 \\
5 \\
5 \\
5\end{array}$ \\
\hline Csv-3 & $12-20-85$ & USGS, & Santa Barbara & $\begin{array}{l}\text { Natural gamms } \\
\text { Caliper } \\
\text { Spontaneous potential } \\
\text { Resistivity, long-normal } \\
\text { Resigtivity, short-normal } \\
\text { Single-point resistence }\end{array}$ & $\begin{array}{r}0 \\
0 \\
580 \\
580 \\
580 \\
580\end{array}$ & $\begin{array}{l}-760 \\
-760 \\
-760 \\
-760 \\
-760 \\
-760\end{array}$ & $\begin{array}{r}9 \\
9 \\
10 \\
10 \\
10 \\
10\end{array}$ \\
\hline & $\begin{array}{l}2-19-86 \\
2-20-86\end{array}$ & USGS, & Denver & $\begin{array}{l}\text { Neutron } \\
\text { Water temperature } \\
\text { Gamma-gamma density }\end{array}$ & $\begin{array}{r}400 \\
590 \\
0\end{array}$ & $\begin{array}{l}-760 \\
-760 \\
-760\end{array}$ & $\begin{array}{l}9 \\
9 \\
9\end{array}$ \\
\hline$C E-D T-4$ & $12-11-80$ & ERTEC & & $\begin{array}{l}\text { Natural gamma } \\
\text { Spontaneous potential } \\
\text { Resiativity }\end{array}$ & $\begin{array}{r}0 \\
353 \\
353\end{array}$ & $\begin{array}{l}-669 \\
-669 \\
-669\end{array}$ & • \\
\hline & $12-04-85$ & USGS, & Denver & $\begin{array}{l}\text { Natural gama } \\
\text { Callper } \\
\text { Water temperature } \\
\text { Neutron } \\
\text { Gamma-gama denolty }\end{array}$ & $\begin{array}{r}10 \\
40 \\
350 \\
10 \\
10\end{array}$ & $\begin{array}{l}-660 \\
-660 \\
-580 \\
-660 \\
-660\end{array}$ & $\begin{array}{l}12 \\
12 \\
12 \\
12 \\
12\end{array}$ \\
\hline CE-DT-5 & $05-06-81$ & ERTEC & & $\begin{array}{l}\text { Natural gamma } \\
\text { Spontaneous potential } \\
\text { Resistivity }\end{array}$ & $\begin{array}{r}0 \\
350 \\
350\end{array}$ & $\begin{array}{l}-628 \\
-628 \\
-628\end{array}$ & * \\
\hline & $11-30-81$ & USGS, & Denver & Water temperature & 350 & -409 & 16 \\
\hline$C E-D T-6$ & $12-05-85$ & USGS, & Denver & $\begin{array}{l}\text { Water temperature } \\
\text { Cal1per }\end{array}$ & $\begin{array}{l}458 \\
320\end{array}$ & $\begin{array}{l}-933 \\
-933\end{array}$ & $\begin{array}{l}20 \\
20\end{array}$ \\
\hline$C E-V F-2$ & $11-22-81$ & ERTEC & & $\begin{array}{l}\text { Natural gamma } \\
\text { Spontaneous potential } \\
\text { Resistivity }\end{array}$ & $\begin{array}{l}0 \\
0 \\
0\end{array}$ & $\begin{array}{l}-1,221 \\
-1,221 \\
-1,221\end{array}$ & * \\
\hline & $\begin{array}{l}12-04-85 \\
12-05-85\end{array}$ & USGS, & Denver & $\begin{array}{l}\text { Water temperature } \\
\text { Natural gamma } \\
\text { Callper } \\
\text { Acoust Ic } \\
\text { Neutron } \\
\text { Gamma-gamma density }\end{array}$ & $\begin{array}{r}600 \\
10 \\
850 \\
844 \\
350 \\
10\end{array}$ & $\begin{array}{l}-1,050 \\
-1,012 \\
-1,007 \\
-1,009 \\
-1,009 \\
-1,008\end{array}$ & $\begin{array}{l}25 \\
25 \\
25 \\
25 \\
25 \\
25\end{array}$ \\
\hline SHV-1 & $12-30-85$ & USGS, & Santa Barbara & $\begin{array}{l}\text { Natural gamma } \\
\text { Single-point resistance }\end{array}$ & $\begin{array}{r}0 \\
820\end{array}$ & $\begin{array}{l}-890 \\
-890\end{array}$ & $\begin{array}{l}28 \\
28\end{array}$ \\
\hline & $\begin{array}{l}02-20-86 \\
02-22-86\end{array}$ & USGS, & Denver & $\begin{array}{l}\text { Water temperature } \\
\text { Neutron } \\
\text { Gamma-gamma density } \\
\text { Caliper }\end{array}$ & $\begin{array}{r}800 \\
0 \\
0 \\
0\end{array}$ & $\begin{array}{l}-890 \\
-890 \\
-866 \\
-890\end{array}$ & $\begin{array}{l}28 \\
28 \\
28 \\
28\end{array}$ \\
\hline
\end{tabular}

1 ERTEC, Ertec Western, Inc.; USGS, U.S. Geologlcal Survey.

2 Asterisk Indicates geophysical well logs available, but not presented in this report. 


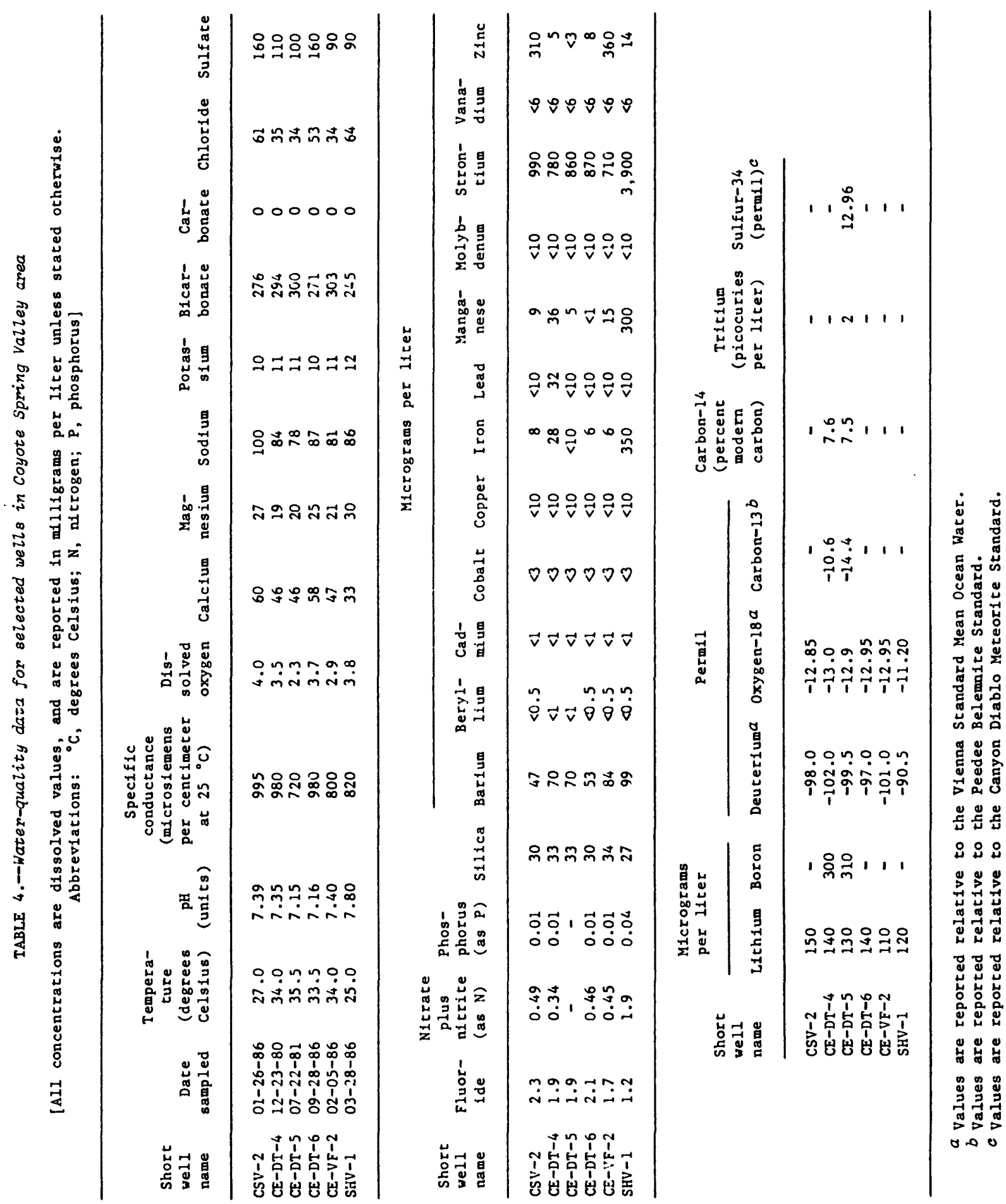




\section{GEOHYDROLOGIC DATA FROM THE EIGHT WELLS}

\section{$\operatorname{CSV}-1$}

We11 CSV-1 is in Moapa Valley, Clark County, and is approximately 1.5 miles south of the intersection of State Route 168 and an abandoned highway (figure 1). The well site is near the intersection of Wildcat Wash and Pahranagat Wash east of the northern edge of the Arrow Canyon Range.

The Geological Survey began drilling on October 10, 1985. The drill penetrated 765 feet of basin-fill sediments and reached ground water at approximately 344 feet below land surface. The drilling penetration rate and lithologic $\log$ are shown in figure 2. A shift in the natural gamma and neutron logs near 550 feet indicated possible bedrock and gave reason to at tempt a core sample from the well bottom; however, no sample was recovered due to core-barrel damage. The well was cased with two polyvinyl chloride (PVC) piezometers: a 2-inch-diameter piezometer and a 4-inch-diameter piezometer (table 2). A bentonite plug was installed at a depth interval of 362 to 369 feet to prevent leakage along the 4-inch casing. The annulus was then filled with clean gravel pack from 362 feet to the surface plug. Geophysical logs for this well are presented in figure 3 . 


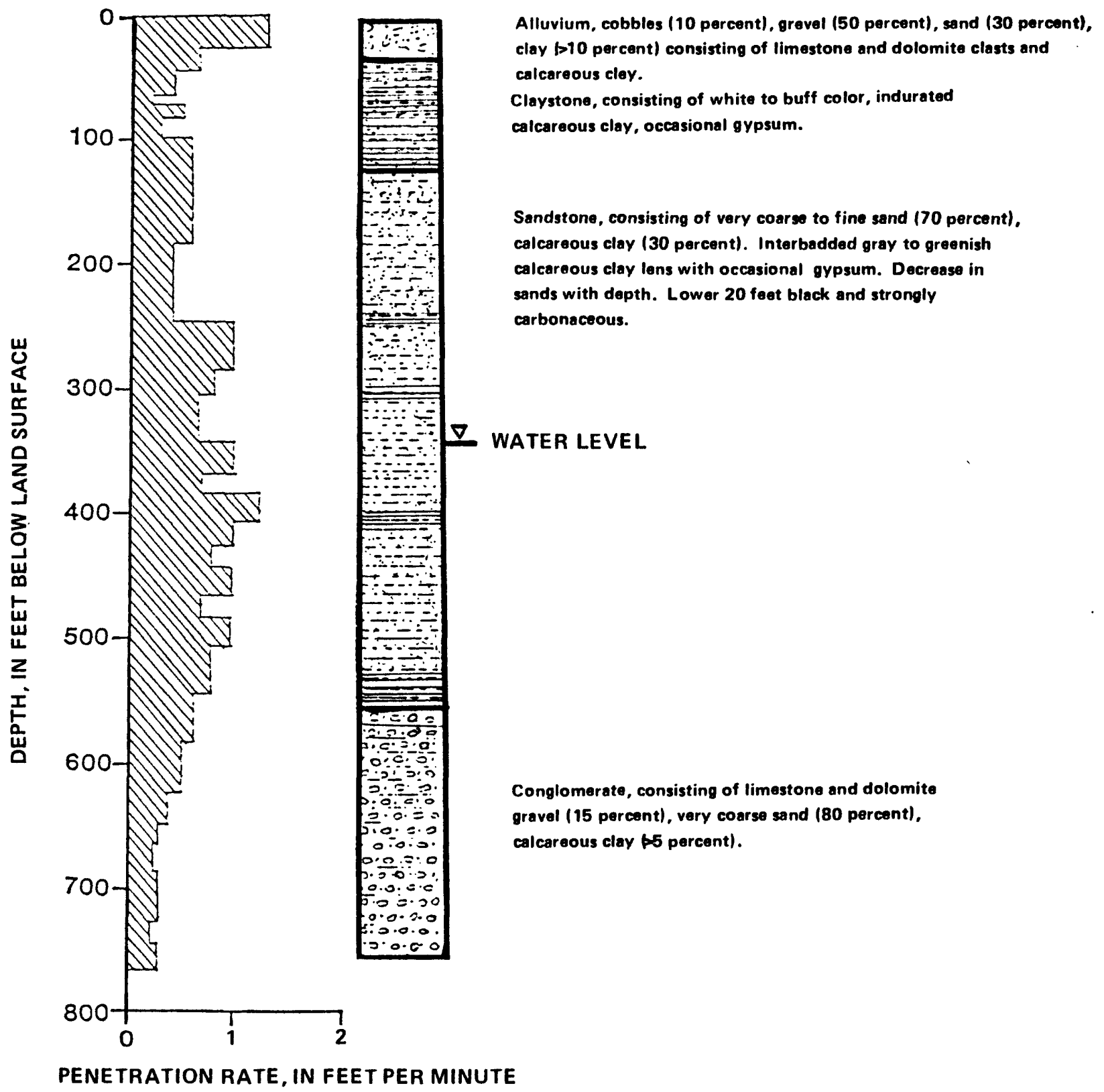

FIGURE 2.--Drilling penetration rate and lithology for U.S. Geological Survey test well CSV-1. 


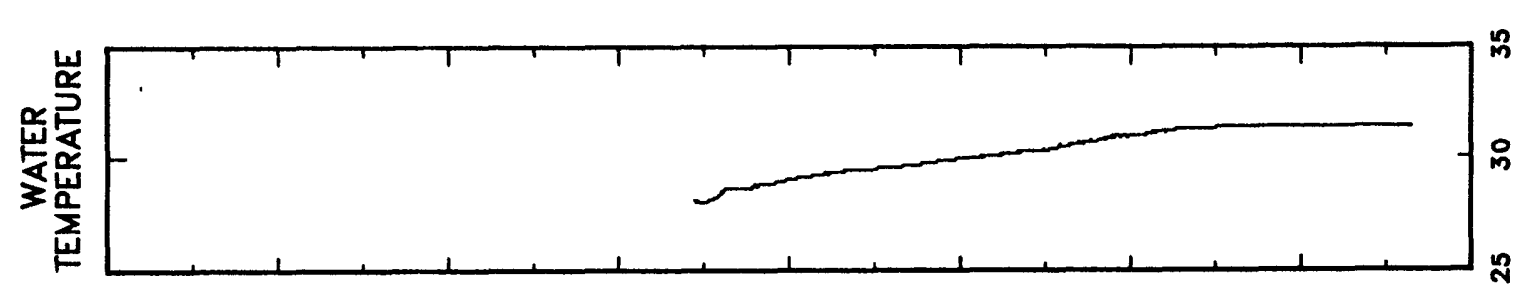

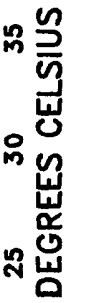
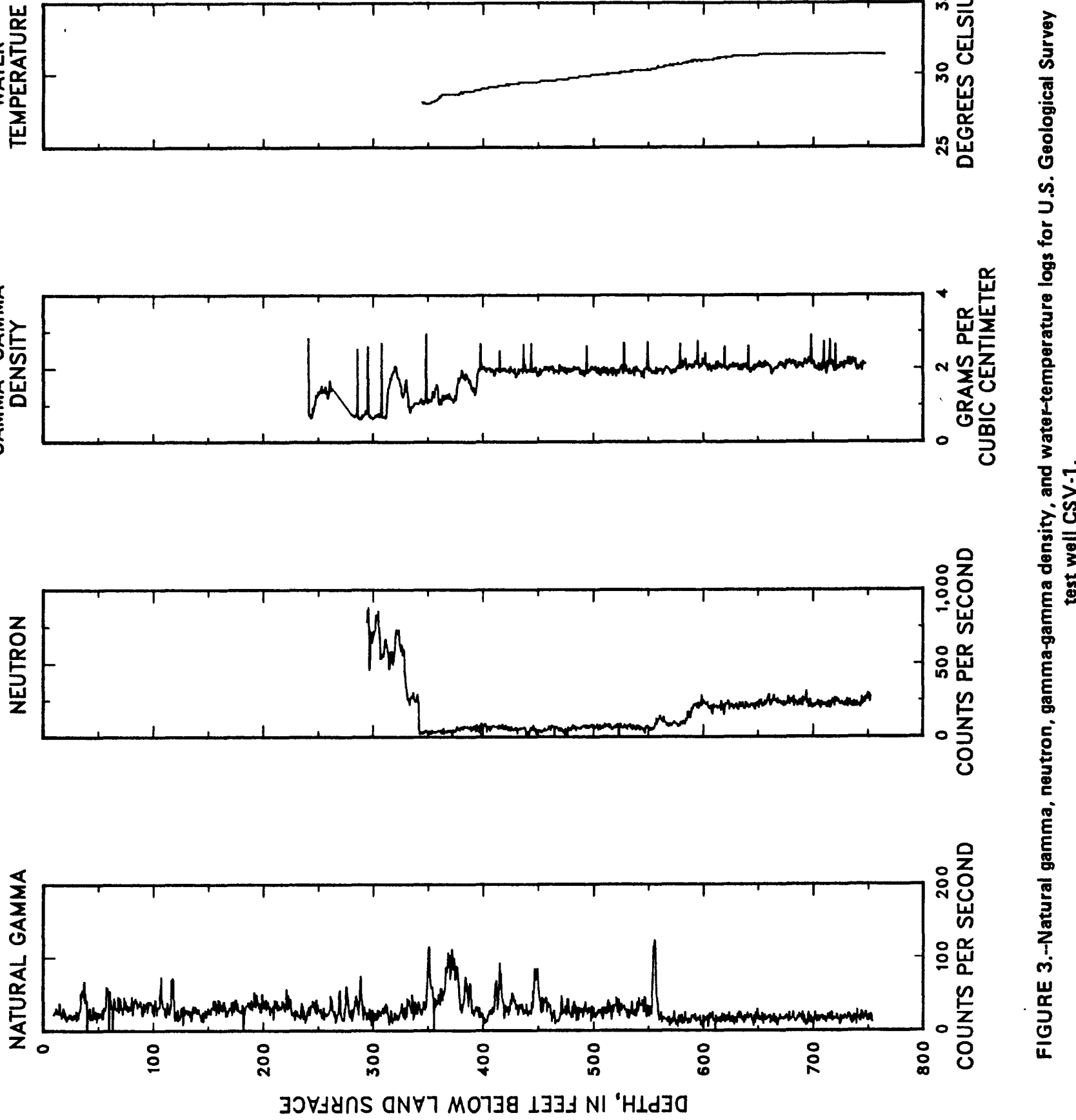


\section{$\mathrm{CSV}-2$}

We11 CSV-2 is in Moapa Valley, Clark County, and is approximately 3.2 miles north of the intersection of State Route 168 and Warm Springs Road (figure 1). The well site is in an unnamed drainage on the southwestern flank of the Meadow Valley Mountains and south of Farrier Wash.

The U.S. Geological Survey began drilling on October 23, 1985. Limestone bedrock was penetrated at 17 feet and the water table was reached at approximately 390 feet below land surface. Drilling continued through the limestone section until october 26, when the 8-footlong drill bit and stem broke of $f$ at a depth of 478 feet, after penetrating a large fracture and dropping several feet. Numerous attempts to retrieve the bit and stem were unsuccessful. The well was cased to bedrock with 10-inch-diameter PVC. The drilling penetration rate and lithologic $\log$ are shown in figure 4. Geophysical logs are presented in figure 5.

An aquifer test for CSV-2 was made by the Geological Survey in June 1986. Prior to this test, the well was developed by pumping for 2 days at approximately 5,500 gal/d. A 20-horsepower, 6-inch-diameter submersible pump with a 3-inch-diameter discharge pipe was used for the test. The intake was set at 430 feet. A combined total-discharge and sweep-hand flow meter was installed in-line to measure well discharge. Discharge was piped 60 feet from the well to a small wash that transported flow from the site. Water levels were measured in the well with a recording pressure transducer set at 420 feet and calibrated on site. The drawdown test lasted 21 hours with a constant discharge of about $100 \mathrm{gal} / \mathrm{min}$. Total recovery was observed within 1 hour of stopping the pump. Tables 5 and 6 and figures 6 and 7 present the test data. 


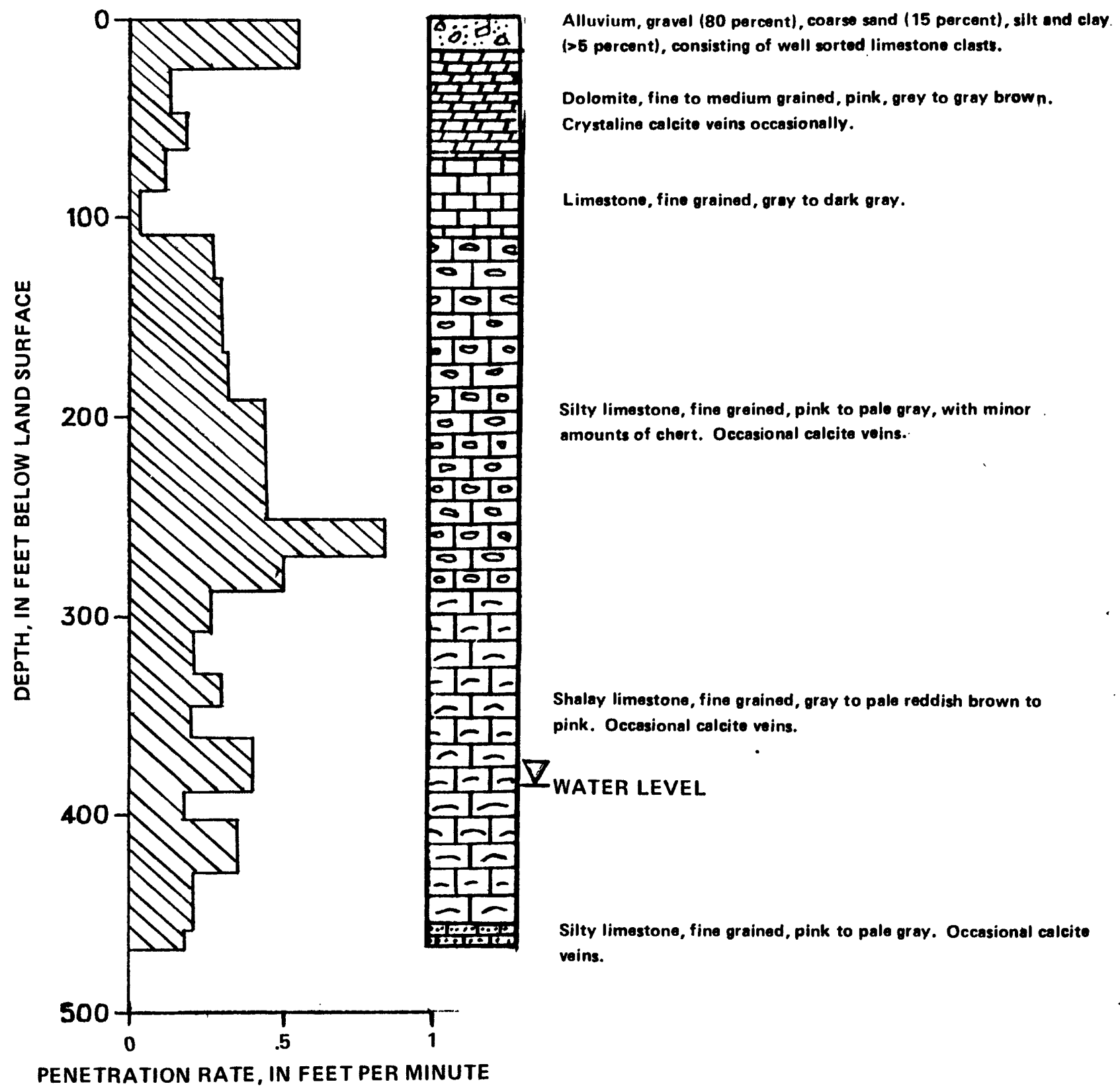

FIGURE 4.--Drilling penetration rate and lithology for U.S. Geological Survey test well CSV-2. 

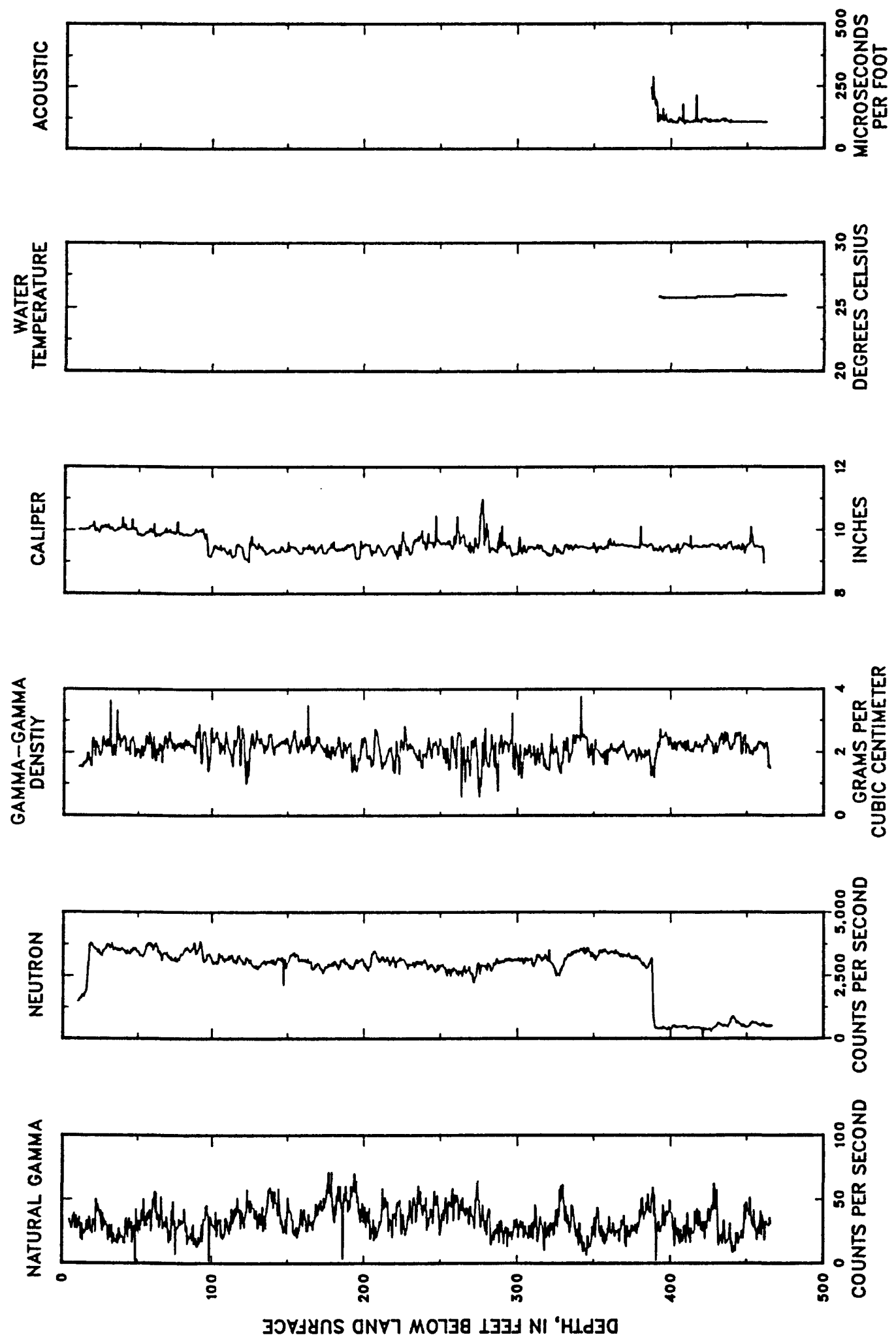
TABLE 5.--Water levels during a 21.5 -hour, constant-discharge aquifer test at IJ.S. Geological Survey test well CSV-2, June 7-8, 1986. Static water level is 390.76

[TIme, minutes since pumping began; water leve1, feet below land surface; drawdown, calculated drawdown of water levels in feet below static water level; pumping rate, spot-checked during test (average pumping rate from flow totallzer was 100.5 gallons per minute)]

\begin{tabular}{|c|c|c|c|c|c|c|c|}
\hline $\begin{array}{c}\text { Time } \\
\text { (minutes) }\end{array}$ & $\begin{array}{l}\text { Water } \\
\text { leve } 1 \\
\text { (feet) }\end{array}$ & $\begin{array}{l}\text { Draw- } \\
\text { down } \\
\text { (feet) }\end{array}$ & $\begin{array}{l}\text { Pumping rate } \\
\text { (gallons } \\
\text { per minute) }\end{array}$ & $\begin{array}{c}\text { Time } \\
\text { (minutes) }\end{array}$ & $\begin{array}{l}\text { Water } \\
\text { leve } 1 \\
\text { (feet) }\end{array}$ & $\begin{array}{c}\text { Draw- } \\
\text { down } \\
(\text { feet })\end{array}$ & $\begin{array}{l}\text { Pumping rate } \\
\text { (gallons } \\
\text { per minute) }\end{array}$ \\
\hline 0.00 & 390.8 & 0.0 & Pump on & 0.52 & 400.7 & 9.9 & \\
\hline 0.01 & 392.0 & 1.2 & 100 & 0.59 & 401.6 & 10.8 & \\
\hline 0.03 & 391.4 & 0.6 & & 0.66 & 402.3 & 11.5 & \\
\hline 0.04 & 391.9 & 1.1 & & 0.74 & 403.0 & 12.2 & \\
\hline 0.06 & 392.3 & 1.5 & & 0.81 & 403.7 & 12.9 & \\
\hline 0.07 & 392.7 & 1.9 & & 0.88 & 404.2 & 13.4 & ' \\
\hline 0.09 & 393.1 & 2.3 & & 0.96 & 404.8 & 14.0 & \\
\hline 0.10 & 393.5 & 2.7 & & 1.03 & 405.3 & 14.5 & 100 \\
\hline 0.12 & 393.9 & 3.1 & & 1.11 & 405.8 & 15.0 & \\
\hline 0.13 & 394.3 & 3.5 & & 1.18 & 406.2 & 15.4 & \\
\hline 0.16 & 394.9 & 4.1 & & 1.25 & 406.7 & 15.9 & \\
\hline 0.18 & 395.3 & 4.5 & & 1.33 & 407.1 & 16.3 & \\
\hline 0.19 & 395.6 & 4.8 & & 1.40 & 407.4 & 16.6 & \\
\hline 0.21 & 395.9 & 5.1 & & 1.47 & 407.8 & 17.0 & \\
\hline 0.22 & 396.1 & 5.3 & & 1.55 & 408.1 & $17 \cdot 3$ & \\
\hline 0.24 & 396.4 & 5.6 & & 1.62 & 408.4 & 17.6 & \\
\hline 0.25 & 396.7 & 5.9 & & 1.70 & 408.6 & 17.8 & \\
\hline 0.26 & 397.0 & 6.2 & & 1.77 & 408.9 & 18.1 & \\
\hline 0.28 & 397.2 & 6.4 & & 1.84 & 409.2 & 18.4 & \\
\hline 0.29 & 397.5 & 6.7 & & 1.92 & 409.4 & 18.6 & \\
\hline 0.31 & 397.8 & 7.0 & & 1.99 & 409.7 & 18.9 & \\
\hline 0.32 & 398.0 & 7.2 & & 2.06 & 409.9 & 19.1 & \\
\hline 0.34 & 398.2 & 7.4 & & 2.14 & 410.1 & 19.3 & \\
\hline 0.35 & 398.5 & 7.7 & & 2.21 & 410.3 & 19.5 & \\
\hline 0.37 & 398.7 & 7.9 & & 2.29 & 410.4 & 19.6 & \\
\hline 0.38 & 398.9 & 8.1 & & 2.36 & 410.5 & 19.7 & \\
\hline 0.40 & 399.1 & 8.3 & & 2.43 & 410.7 & 19.9 & \\
\hline 0.41 & 399.3 & 8.5 & & 2.51 & 410.8 & 20.0 & \\
\hline 0.43 & 399.5 & 8.7 & & 2.58 & 410.9 & 20.1 & \\
\hline 0.44 & 399.7 & 8.9 & & 2.65 & 411.1 & 20.3 & \\
\hline
\end{tabular}


TABLE 5.--Water levels during a 21.b-hour, constant-discharge aquif'er test at U.S. Geological Survey test well CSV-2, June 7-8, 1986--Cont inued

\begin{tabular}{|c|c|c|c|c|c|c|c|}
\hline $\begin{array}{c}\text { Time } \\
\text { (minutes) }\end{array}$ & $\begin{array}{l}\text { Water } \\
\text { leve } 1 \\
\text { (feet) }\end{array}$ & $\begin{array}{l}\text { Draw- } \\
\text { down } \\
\text { (feet) }\end{array}$ & $\begin{array}{l}\text { Pumping rate } \\
\text { (gallons } \\
\text { per minute) }\end{array}$ & $\begin{array}{c}\text { Time } \\
\text { (minutes) }\end{array}$ & $\begin{array}{l}\text { Water } \\
\text { leve } 1 \\
\text { (feet) }\end{array}$ & $\begin{array}{l}\text { Draw- } \\
\text { down } \\
\text { (feet) }\end{array}$ & $\begin{array}{l}\text { Pumping rate } \\
\text { (gallons } \\
\text { per minute) }\end{array}$ \\
\hline $\begin{array}{l}3.10 \\
3.54\end{array}$ & $\begin{array}{l}411.8 \\
412.4\end{array}$ & $\begin{array}{l}21.0 \\
21.6\end{array}$ & & $\begin{array}{l}29.59 \\
34.01\end{array}$ & $\begin{array}{l}417.1 \\
417.4\end{array}$ & $\begin{array}{l}26.3 \\
26.6\end{array}$ & 100 \\
\hline 3.98 & 412.9 & 22.1 & & 38.44 & 417.6 & 26.8 & \\
\hline 4.42 & 413.3 & 22.5 & & 42.86 & 417.8 & 27.0 & \\
\hline 4.87 & 413.6 & 22.8 & & 47.29 & 417.9 & 27.1 & \\
\hline $\begin{array}{l}5.31 \\
5.75\end{array}$ & $\begin{array}{l}413.9 \\
414.2\end{array}$ & $\begin{array}{l}23.1 \\
23.4\end{array}$ & 101 & $\begin{array}{l}51.71 \\
56.14\end{array}$ & $\begin{array}{l}418.0 \\
418.1\end{array}$ & $\begin{array}{l}27.2 \\
27.3\end{array}$ & \\
\hline 6.19 & 414.5 & 23.7 & & 60.56 & 418.2 & 27.4 & 101 \\
\hline $\begin{array}{l}6.64 \\
7.08\end{array}$ & $\begin{array}{l}414.7 \\
415.0\end{array}$ & $\begin{array}{l}23.9 \\
24.2\end{array}$ & & $\begin{array}{l}64.98 \\
69.41\end{array}$ & $\begin{array}{l}418.3 \\
418.4\end{array}$ & $\begin{array}{l}27.5 \\
27.6\end{array}$ & \\
\hline $\begin{array}{l}7.52 \\
7.96\end{array}$ & $\begin{array}{l}415.2 \\
415.4\end{array}$ & $\begin{array}{l}24.4 \\
24.6\end{array}$ & & $\begin{array}{l}73.83 \\
78.26\end{array}$ & $\begin{array}{l}418.4 \\
418.5\end{array}$ & $\begin{array}{l}27.6 \\
27.7\end{array}$ & 、 \\
\hline 8.41 & 415.6 & 24.8 & & 82.68 & 418.5 & 27.7 & \\
\hline 8.85 & 415.7 & 24.9 & & 87.11 & 418.6 & 27.8 & \\
\hline 9.29 & 415.8 & 25.0 & & 91.53 & 418.6 & 27.8 & 103 \\
\hline 9.73 & 416.0 & 25.2 & & 104.81 & 418.7 & 27.9 & \\
\hline 10.18 & 416.1 & 25.3 & 100 & 11.8 .08 & 418.9 & 28.1 & 101 \\
\hline 10.62 & 416.2 & 25.4 & & 150.00 & 419.0 & 28.2 & 101 \\
\hline 11.06 & 416.2 & 25.4 & & 180.00 & 419.2 & 28.4 & 102 \\
\hline 11.50 & 416.3 & 25.5 & & 210.00 & 419.5 & 28.7 & 101 \\
\hline $\begin{array}{l}11.89 \\
12.77\end{array}$ & $\begin{array}{l}416.4 \\
416.5\end{array}$ & $\begin{array}{l}25.6 \\
25.7\end{array}$ & & $\begin{array}{l}240.00 \\
270.00\end{array}$ & $\begin{array}{l}419.6 \\
419.9\end{array}$ & $\begin{array}{l}28.8 \\
29.1\end{array}$ & 102 \\
\hline 13.66 & 416.6 & 25.8 & & 300.00 & 419.9 & 29.1 & 99 \\
\hline 14.54 & 416.7 & 25.9 & & 330.00 & 420.0 & 29.2 & 100 \\
\hline 15.43 & 416.8 & 26.0 & 103 & 360.00 & 420.0 & 29.2 & 101 \\
\hline $\begin{array}{l}16.31 \\
17.20\end{array}$ & $\begin{array}{l}416 \cdot 9 \\
417.0\end{array}$ & $\begin{array}{l}26.1 \\
26.2\end{array}$ & & $\begin{array}{l}390.00 \\
420.00\end{array}$ & $\begin{array}{l}420 \cdot 1 \\
420 \cdot 2\end{array}$ & $\begin{array}{l}29.3 \\
29.4\end{array}$ & $\begin{array}{l}101 \\
100\end{array}$ \\
\hline 18.08 & 417.1 & 26.3 & & 423.00 & 420.8 & 30.0 & pump of $f$ \\
\hline 18.97 & 417.1 & 26.3 & & & & & 30 seconds \\
\hline 19.85 & 417.2 & 26.4 & & 440.00 & $420 \cdot 5$ & 29.7 & 99 \\
\hline 20.74 & 417.2 & 26.4 & & 480.00 & 420.6 & 29.8 & 99 \\
\hline 22.95 & 416.8 & 26.0 & & 510.00 & 420.4 & 29.6 & 103 \\
\hline 25.16 & 416.8 & 26.0 & 100 & 540.00 & 420.5 & 29.7 & 101 \\
\hline 27.37 & 417.0 & 26.2 & & 570.00 & 420.5 & 29.7 & 102 \\
\hline
\end{tabular}


TABLE 5.--Water levels during a 21.5-hour, constant-discharge aquifer test at U.S. Geological Survey test well CSV-2, June 7-8, 1986--Continued

\begin{tabular}{|c|c|c|c|c|c|c|c|}
\hline $\begin{array}{c}\text { Time } \\
\text { (minutes) }\end{array}$ & $\begin{array}{l}\text { Water } \\
\text { leve } 1 \\
\text { (feet) }\end{array}$ & $\begin{array}{l}\text { Draw- } \\
\text { down } \\
\text { (feet) }\end{array}$ & $\begin{array}{l}\text { Pumplng rate } \\
\text { (gallons } \\
\text { per minute) }\end{array}$ & $\begin{array}{c}\text { Time } \\
\text { (minutes) }\end{array}$ & $\begin{array}{l}\text { Water } \\
\text { level } \\
\text { (feet) }\end{array}$ & $\begin{array}{l}\text { Draw- } \\
\text { down } \\
\text { (feet) }\end{array}$ & $\begin{array}{l}\text { Pumping rate } \\
\text { (gallons } \\
\text { per minute) }\end{array}$ \\
\hline 600.00 & 420.5 & 29.7 & 99 & 960.00 & 420.9 & 30.1 & 101 \\
\hline 630.00 & 420.3 & 29.5 & 101 & 990.00 & 420.8 & 30.0 & 100 \\
\hline 660.00 & 420.3 & 29.5 & 99 & 1020.00 & 420.8 & 30.0 & 101 \\
\hline 690.00 & 420.6 & 29.8 & 100 & 1050.00 & 420.8 & 30.0 & 100 \\
\hline 720.00 & 420.8 & 30.0 & 99 & 1080.00 & 420.8 & 30.0 & 101 \\
\hline 750.00 & 420.8 & 30.0 & 100 & 1110.00 & 420.7 & 29.9 & 100 \\
\hline 780.00 & 420.6 & 29.8 & 100 & 1140.00 & 420.6 & 29.8 & 101 \\
\hline 810.00 & 420.6 & 29.8 & 101 & 1170.00 & 420.4 & 29.6 & 101 \\
\hline 840.00 & 420.6 & 29.8 & 100 & 1200.00 & 420.5 & 29.7 & 100 \\
\hline 870.00 & 420.8 & 30.0 & 100 & 1230.00 & 420.6 & 29.8 & 101 \\
\hline 900.00 & 420.8 & 30.0 & 101 & 1260.00 & 420.6 & 29.8 & 100 \\
\hline 930.00 & 420.9 & 30.1 & 102 & 1290.00 & 420.6 & 29.8 & pump off \\
\hline
\end{tabular}

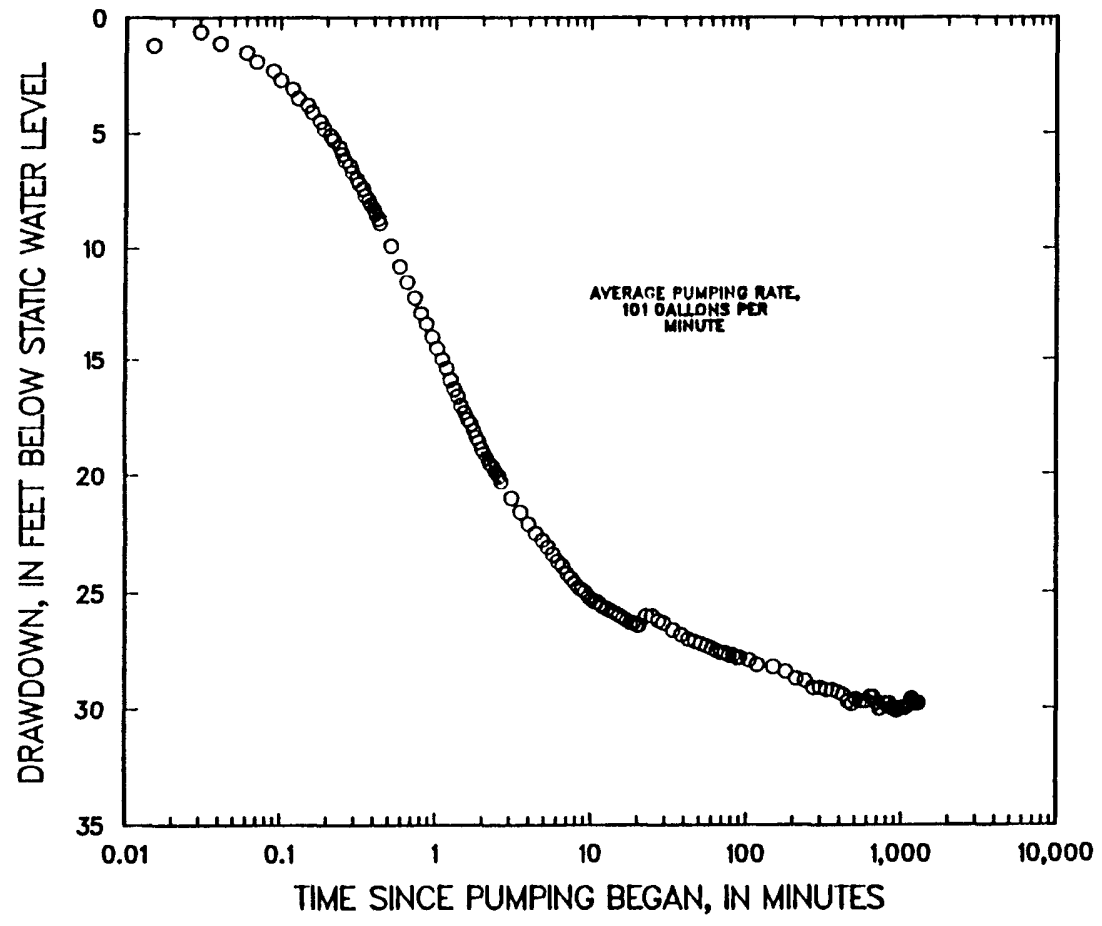

FIGURE 6.--Drawdown versus time at U.S. Geological Survey test well CSV-2, June 7-8, 1986. 
TABLE 6.--Water levels during recovery from a 21.5-hour, constant-discharge aquifer test at U.S. Geological survey test well CisV-2, dune 8, 1986

[Time, minutes since pumping ended; water level, feet below land surface; resldual drawdown, calculated drawdown of water levels remaining below static water level]

\begin{tabular}{|c|c|c|c|c|c|}
\hline $\begin{array}{c}\text { T1me } \\
\text { (minutes) }\end{array}$ & $\begin{array}{l}\text { Water } \\
\text { level } \\
\text { (feet) }\end{array}$ & $\begin{array}{c}\text { Residual } \\
\text { drawdown } \\
\text { (feet) }\end{array}$ & $\begin{array}{c}\text { Time } \\
\text { (minutes) }\end{array}$ & $\begin{array}{l}\text { Water } \\
\text { leve1 } \\
\text { (feet) }\end{array}$ & $\begin{array}{l}\text { Res Idual } \\
\text { drawdown } \\
\text { (feet) }\end{array}$ \\
\hline 0.02 & 420.3 & 29.5 & 0.59 & 403.4 & 12.6 \\
\hline 0.03 & 419.7 & 28.9 & 0.66 & 401.7 & 10.9 \\
\hline 0.04 & 419.2 & 28.4 & 0.74 & 400.2 & 9.4 \\
\hline 0.06 & 418.6 & 27.8 & 0.81 & 398.8 & 8.0 \\
\hline 0.07 & 418.2 & 27.4 & 0.88 & 397.6 & 6.8 \\
\hline 0.09 & 417.6 & 26.8 & 0.96 & 396.5 & 5.7 \\
\hline 0.10 & 417.1 & 26.3 & 1.03 & 395.5 & 4.7 \\
\hline 0.13 & 416.5 & 25.7 & 1.11 & 394.6 & 3.8 \\
\hline 0.15 & 416.0 & 25.2 & 1.18 & 393.9 & 3.1 \\
\hline 0.16 & 415.6 & 24.8 & 1.25 & 393.3 & 2.5 \\
\hline 0.18 & 415.0 & 24.2 & 1.33 & 392.8 & 2.0 \\
\hline 0.19 & 414.5 & 23.7 & 1.40 & 392.4 & 1.6 \\
\hline 0.21 & 414.1 & 23.3 & 1.47 & 392.2 & 1.4 \\
\hline 0.22 & 413.6 & 22.8 & 1.55 & 392.0 & 1.2 \\
\hline 0.24 & 413.2 & 22.4 & 1.62 & 391.8 & 1.0 \\
\hline 0.25 & 412.7 & 21.9 & 1.70 & 391.7 & 0.9 \\
\hline 0.27 & 412.2 & 21.4 & 1.77 & 391.7 & 0.9 \\
\hline 0.28 & 411.7 & 20.9 & 1.92 & 391.6 & 0.8 \\
\hline 0.29 & 411.3 & 20.5 & 2.06 & 391.5 & 0.7 \\
\hline 0.31 & 410.8 & 20.0 & 2.21 & 391.5 & 0.7 \\
\hline 0.32 & 410.4 & 19.6 & 2.36 & 391.4 & 0.6 \\
\hline 0.34 & 409.9 & 19.1 & 2.51 & 391.4 & 0.6 \\
\hline 0.35 & 409.5 & 18.7 & 2.65 & 391.4 & 0.6 \\
\hline 0.37 & 409.1 & 18.3 & 3.10 & 391.3 & 0.5 \\
\hline 0.38 & 408.7 & 17.9 & 3.54 & 391.2 & 0.4 \\
\hline 0.40 & 408.2 & 17.4 & 3.98 & 391.2 & 0.4 \\
\hline 0.41 & 407.8 & 17.0 & 4.42 & 391.2 & 0.4 \\
\hline 0.43 & 407.5 & 16.7 & 4.87 & 391.1 & 0.3 \\
\hline 0.44 & 407.1 & 16.3 & 5.31 & 391.1 & 0.3 \\
\hline 0.52 & 405.1 & 14.3 & 6.19 & 391.1 & 0.3 \\
\hline
\end{tabular}


TABLE 6.--Water levels during recovery from a 21.5-hour, constant-discharge ayuifer test at U.S. Geological Survey test well CSV-2, June 8, 1986--Continued

\begin{tabular}{rccccc}
\hline $\begin{array}{c}\text { T1me } \\
\text { (m1nutes) }\end{array}$ & $\begin{array}{c}\text { Water } \\
\text { leve1 } \\
\text { (feet) }\end{array}$ & $\begin{array}{c}\text { Residual } \\
\text { drawdown } \\
\text { (feet) }\end{array}$ & $\begin{array}{c}\text { T1me } \\
\text { (minutes) }\end{array}$ & $\begin{array}{c}\text { Water } \\
\text { leve1 } \\
\text { (feet) }\end{array}$ & $\begin{array}{c}\text { Residual } \\
\text { drawdown } \\
\text { (feet) }\end{array}$ \\
\hline 7.08 & 391.0 & 0.2 & 24.78 & 390.9 & 0.1 \\
7.96 & 391.0 & 0.2 & 27.43 & 390.9 & 0.1 \\
8.85 & 391.0 & 0.2 & 30.09 & 390.8 & 0.0 \\
11.45 & 391.0 & 0.2 & 32.74 & 390.8 & 0.0 \\
14.16 & 390.9 & 0.1 & 35.40 & 390.8 & 0.0 \\
16.81 & 390.9 & 0.1 & 44.25 & 390.8 & 0.0 \\
19.47 & 390.9 & 0.1 & 53.10 & 390.8 & 0.0 \\
22.12 & 390.9 & 0.1 & 60.03 & 390.8 & 0.0 \\
\hline
\end{tabular}

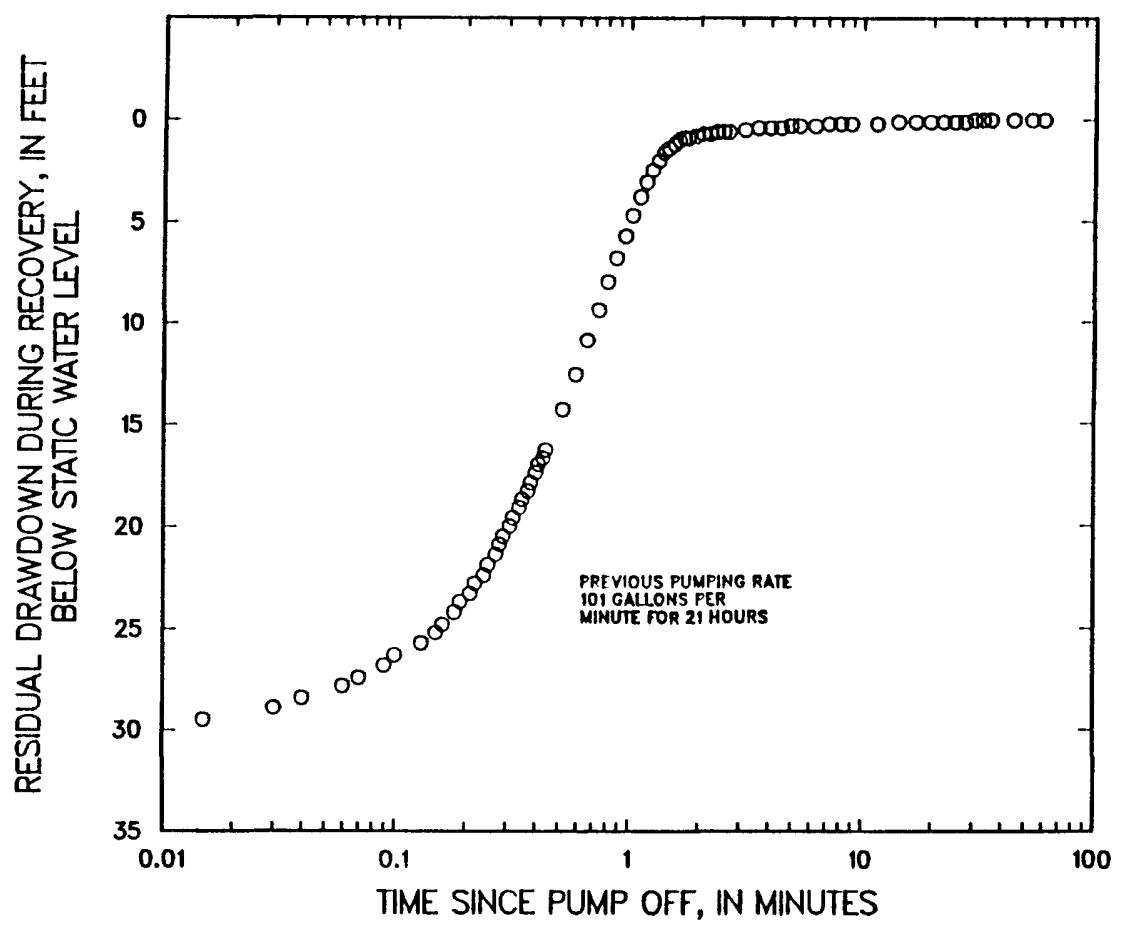

FIGURE 7.--Residual drawdown versus time at U.S. Geological Survey test well CSV-2, June 8, 1986. 


\section{$\underline{\operatorname{csV}-3}$}

We11 CSV-3 in Coyote Spring Valley, Clark County, is adjacent to U.S. Highway 93, approximately 7 miles south of the intersection of U.S. Highway 93 and State Route 168 (figure 1). The well site is in the southernmost part of Coyote Spring Valley, between the Las Vegas Range and the Arrow Canyon Range.

The Geological Survey began drilling on November 6, 1985. Drillingfluid circulation was lost at 430 feet due to a highly permeable and unconsolidated zone identified as a washout on the caliper log. Four thousand gallons of bentonite drilling mud was used as a sealer, but circulation was not regalned. On December 18, 1985, a second episode of drilling was started using a 200-gallon mixture of Suprafoam 1 and Supramud to prevent circulation loss. At 515 feet, fluid circulation was lost which required thickening of the drilling fluid. On December 22, a total depth of 780 feet was attained, penetrating basin-fill deposits. The water table was reached about 580 feet below land surface. The hole later collapsed at a depth of 320 feet, and after reopening, 6-inch PVC was installed to a depth of 756 feet with 20 feet of perforated screen at the bottom. A 10-inch diameter, 7-foot section of PVC surface casing was cemented in place. The drilling penetration rate and 1ithology log are shown in figure 8. Geophysical well logs are shown in figures 9 and 10 .

1 The use of trade or product names in this report is for identification purposes only and does not constitute endorsement by the U.S. Geological Survey. 


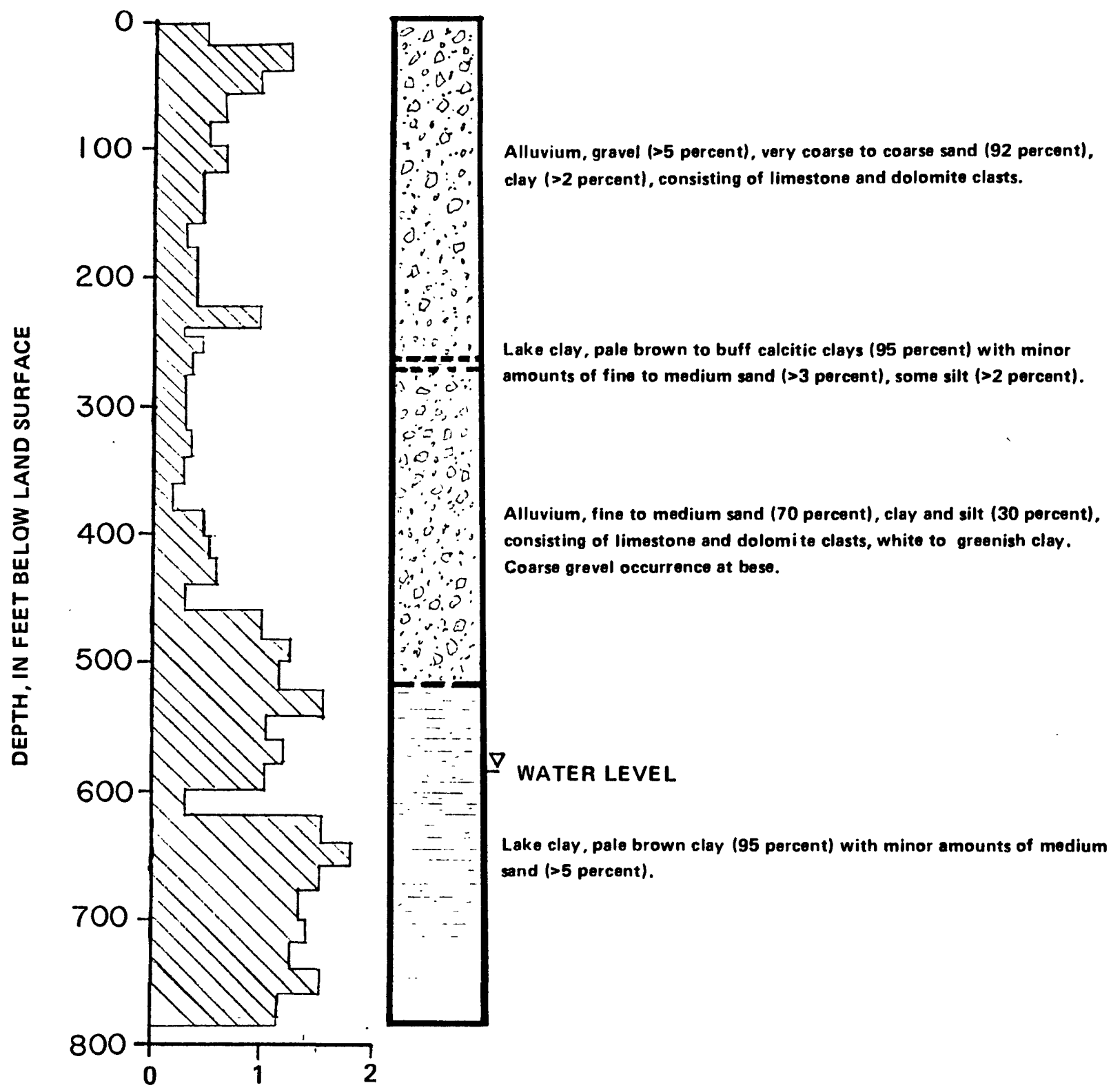

PENETRATION RATE, IN FEET PER MINUTE

FIGURE 8.--Drilling penetration rate and lithology for U.S. Geological Survey test well CSV-3. 


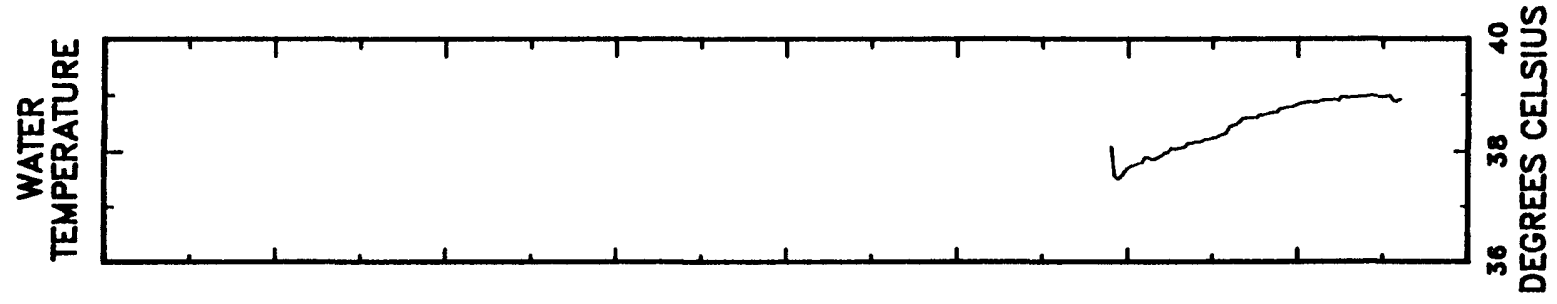

资
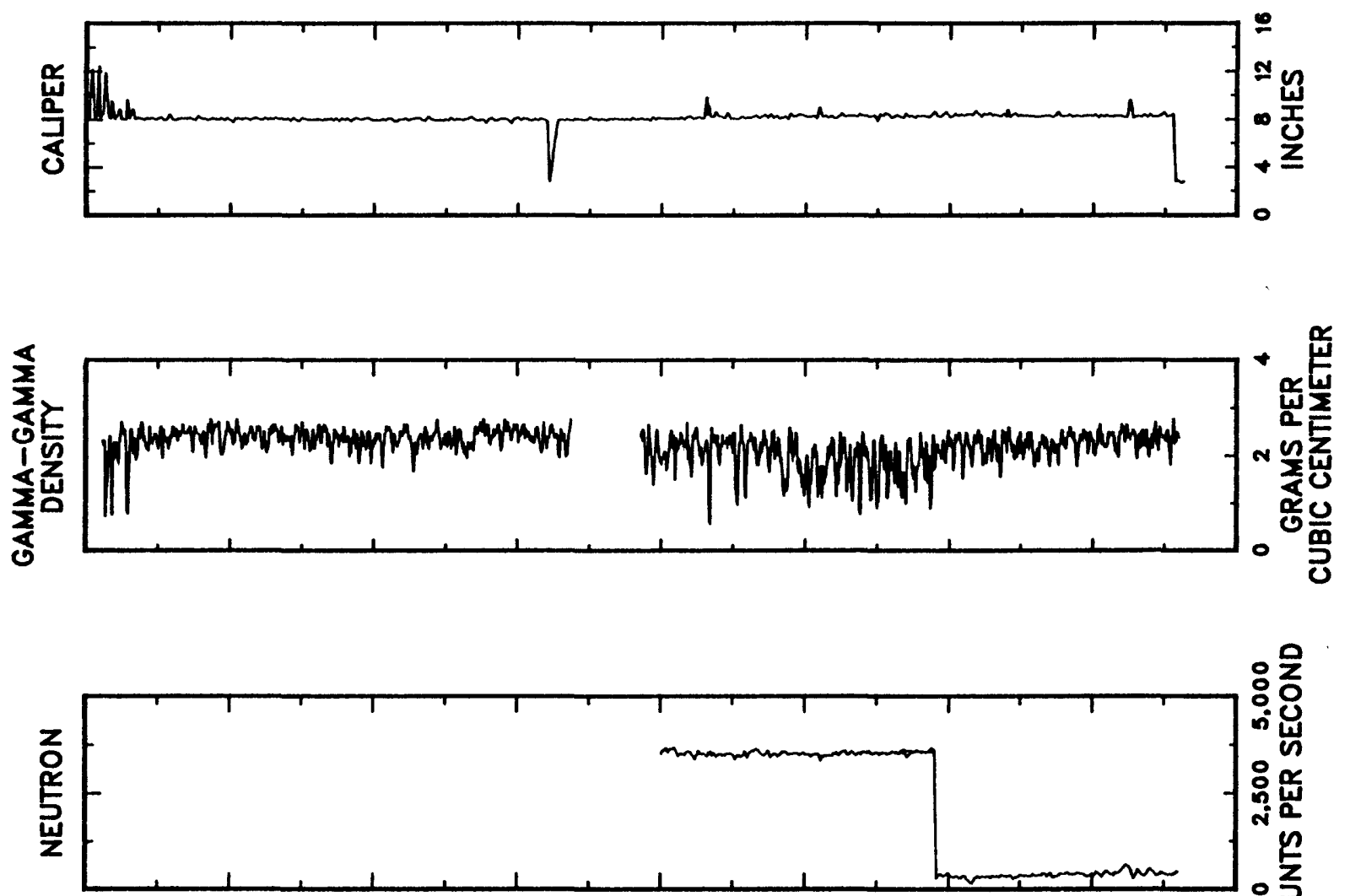

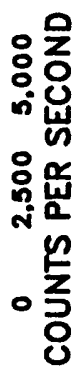

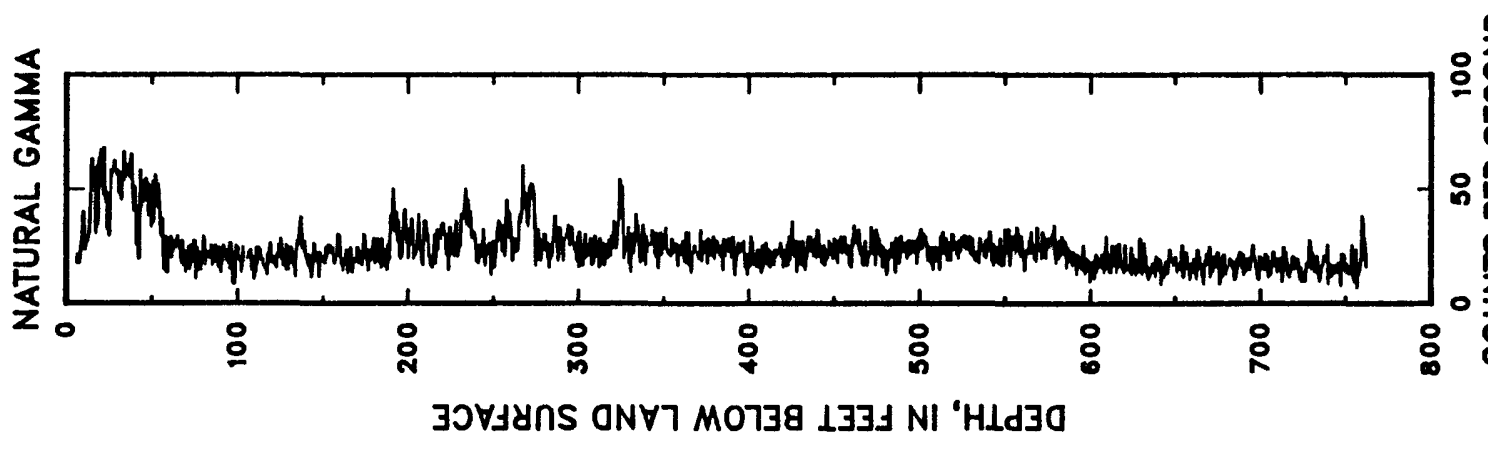

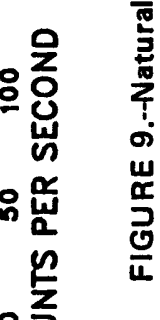



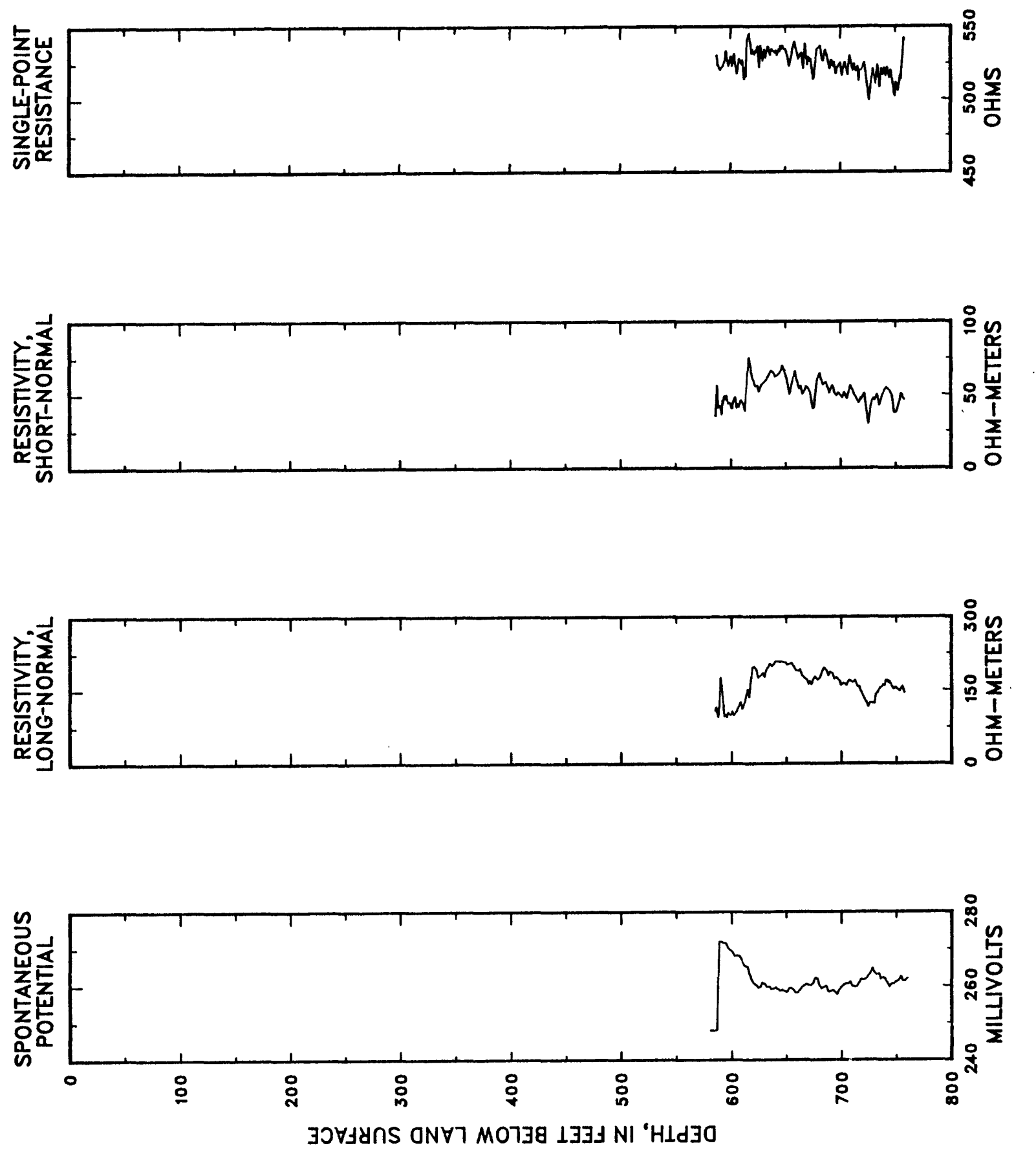


$$
\underline{\text { CE-DT-4 }}
$$

Wells CE-DT-4 and CE-DT-5 are in Coyote Spring Valley, Clark County, and are adjacent to state Route 168 approximately 3 miles east of the intersection of U.S. Highway 93 and State Route 168 (figure 1). CE-DT-4 is located approximately 300 feet west of CE-DT-5. The well sites are in the southeasterly draining Pahranagat Wash and just north of the Arrow Canyon Range.

Drilling began on CE-DT-4 on November 20,1980 . Limestone bedrock was penetrated at 30 feet below land surface, and a total depth of 669 feet was attalned on December 6, 1980, when circulation loss terminated the drilling. The water table was reached at 352 feet below land surface. Due to vertical deviation of the well, cement was installed from 20 feet to the surface, drilled with a 7-7/8-inch-diameter milltooth tricone bit, and reamed with a 17-1/2-inch-diameter button bit. Three feet of 16-inch casing was set at the surface to facilitate sampling. Ten-inch-diameter surface casing was installed from 0 to 50 feet; the remainder of the hole was uncased. The drilling penetration rate and lithologic $\log$ are shown in figure 11. Figure 12 shows the geophysical well logs for the hole.

We11 CE-DT-4 was tested in December 1980 by Hydro Search, Inc. The well was developed by surging five times with the drill-rig airlift at $1,100 \mathrm{ft}^{3} / \mathrm{min}$ before a pump was set. Detalls of the pump setup are not available. The test lasted 3 days and 5 hours, and the discharge rate was about $530 \mathrm{gal} / \mathrm{min}$ (figure 13). Recovery occurred within 2 minutes after the pump was turned of $f$ and on $1 y$ a few water-leve 1 measurements were made (f1gure 14). Water-1eve1 drawdowns during pumping and recoveries after the pump stopped are shown in table 7 . CE-DT-4 was also used as an observation well when aquifer tests were made at $C E-D T-5$. 

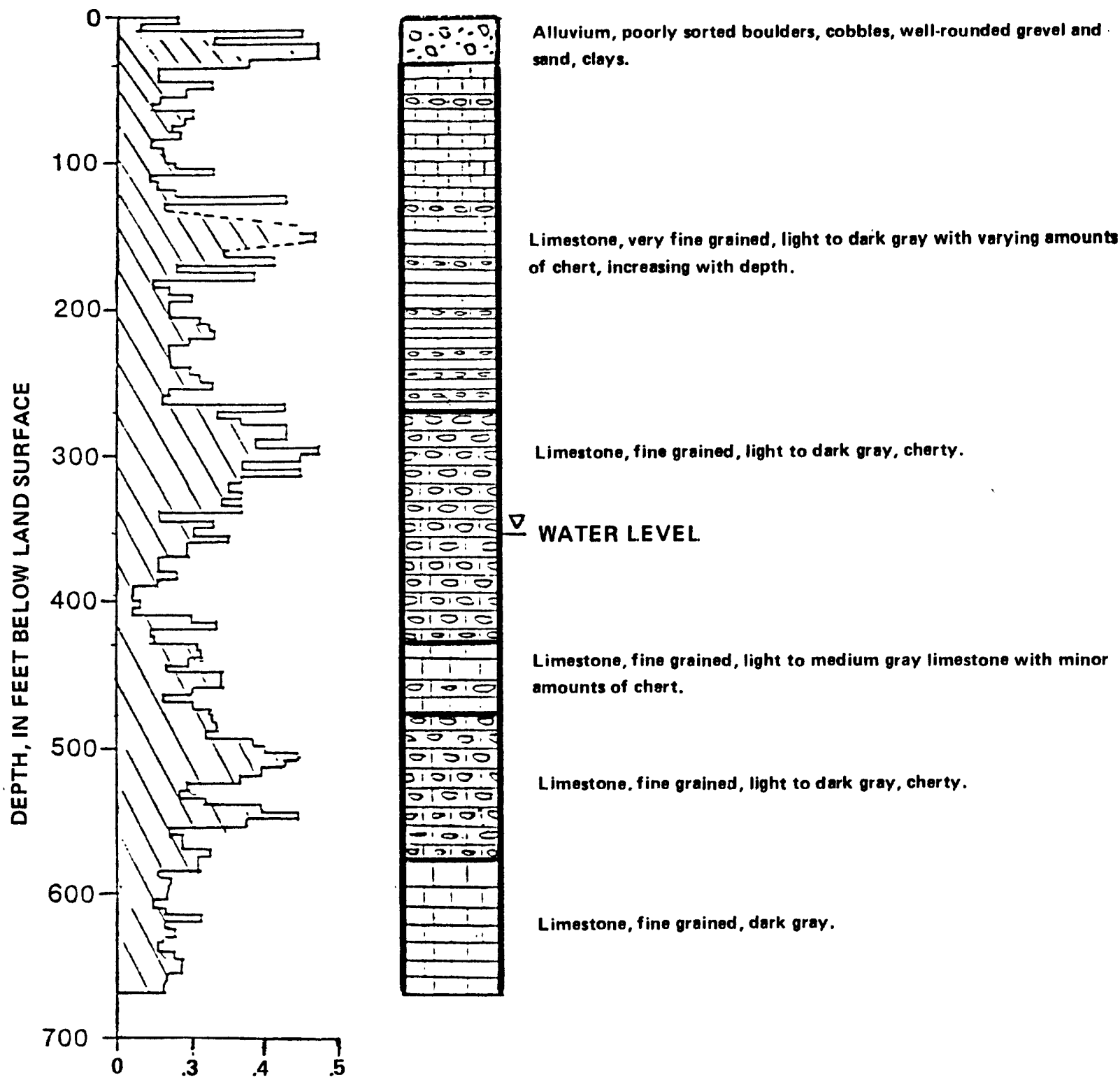

Alluvium, poorly sorted boulders, cobbles, well-rounded grevel and of chert, increasing with depth.

Limestone, fine grained, light to dark gray, cherty.

\section{WATER LEVEL}

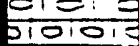

Oा०,

कांड:

Limestone, fine grained, light to medium gray limestone with minor amounts of chart.

Limestone, fine grained, light to dark gray, cherty.

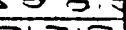

01510

ग1010.0

51010.5

$-10.1<$

गां0.10

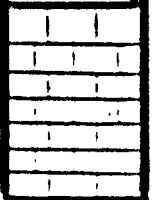

Limestone, fine grained, dark gray.

PENETRATION RATE, IN FEET PER MINUTE

FIGURE 11.--Drilling penetration rate and lithology for MX test well CE-DT-4. 

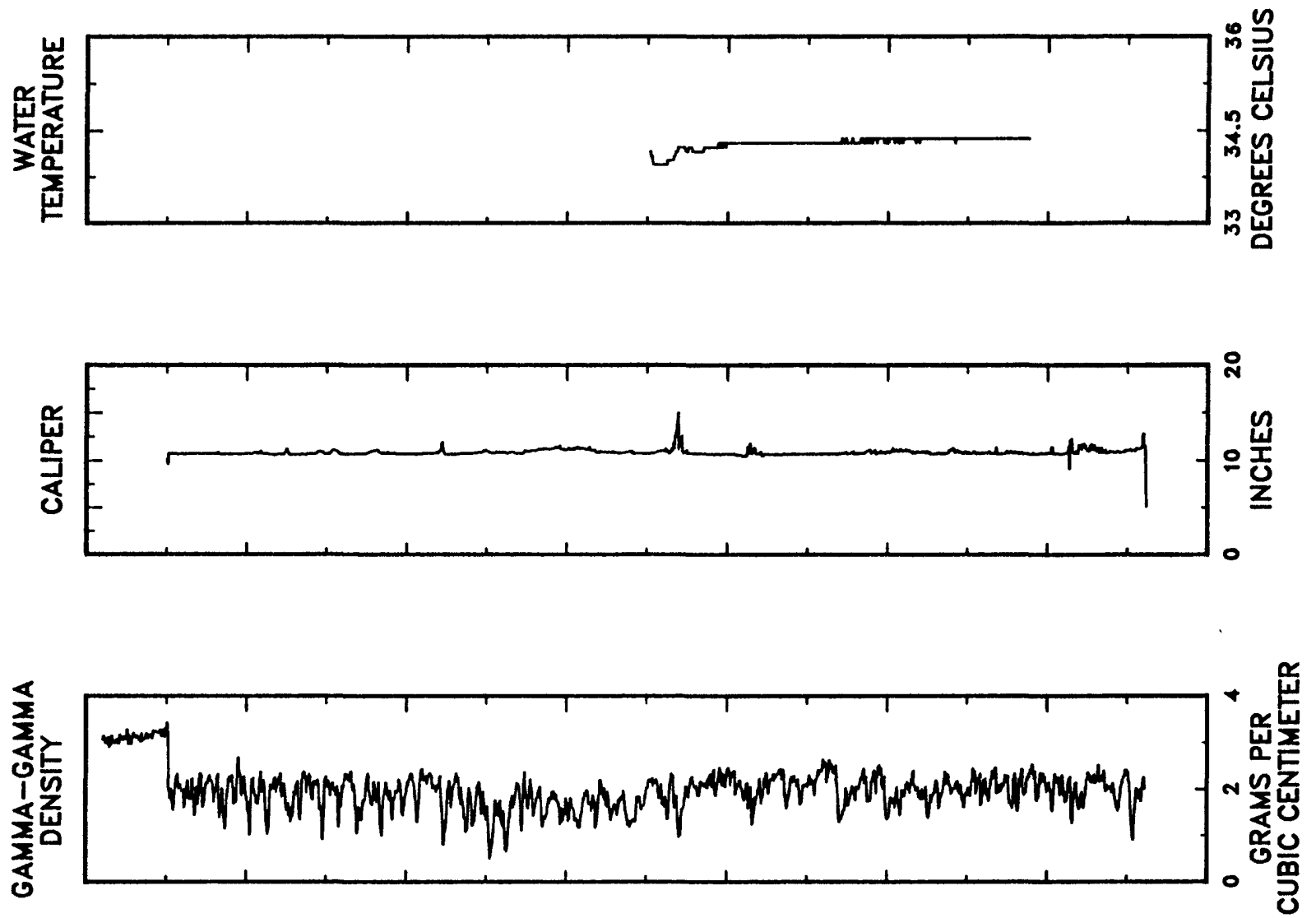

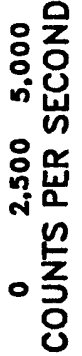

종
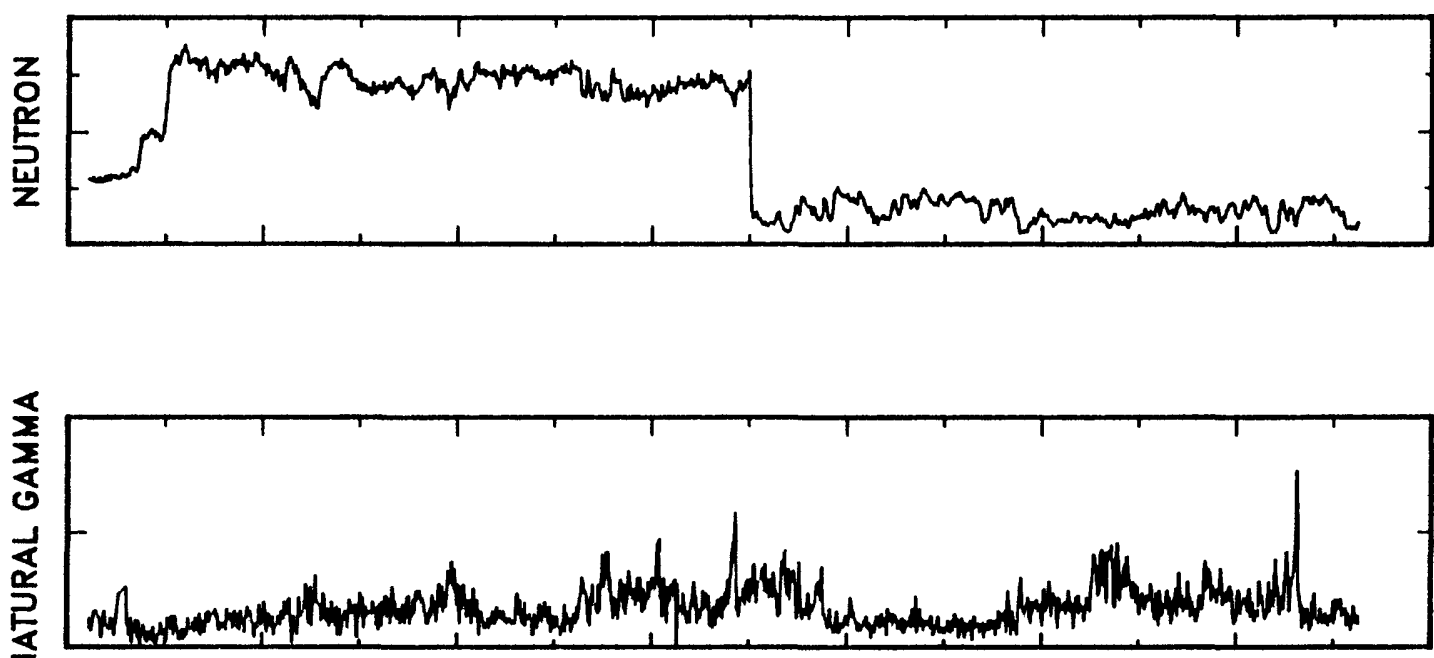

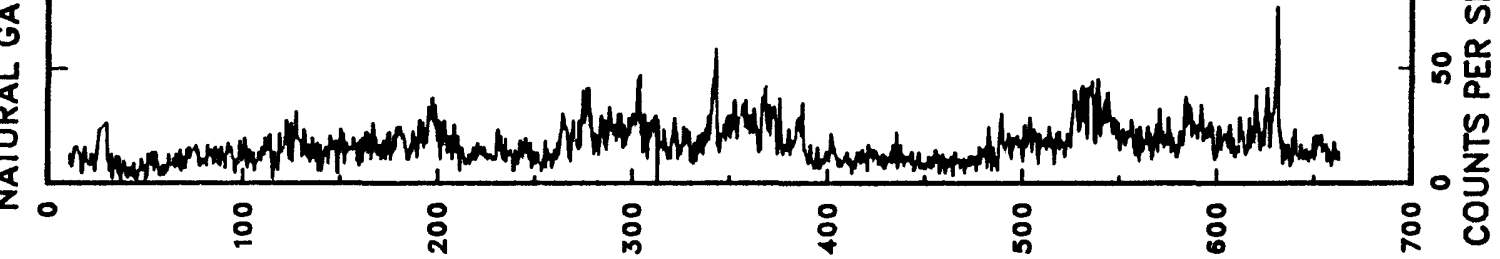

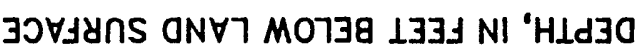




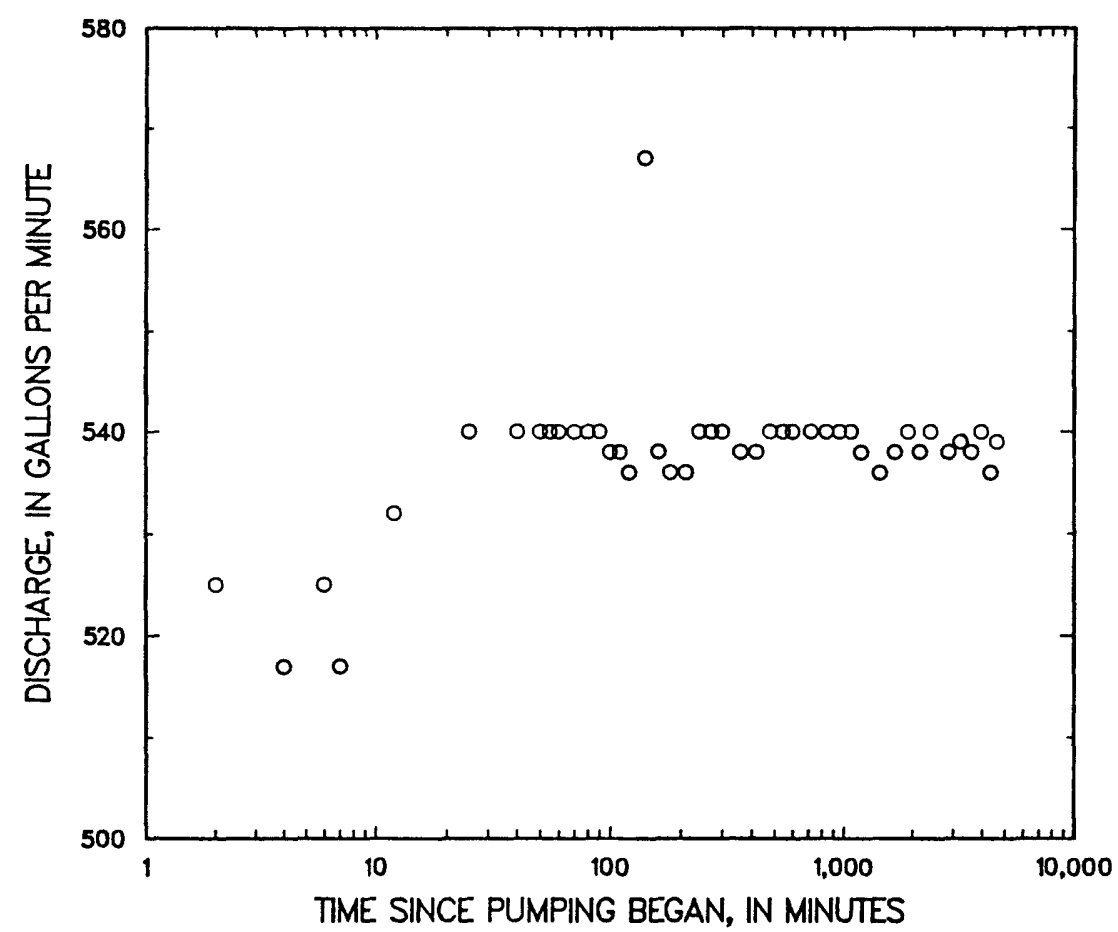

FIGURE 13.--Discharge versus time at MX test well CE-DT-4, December 20-23, 1980.

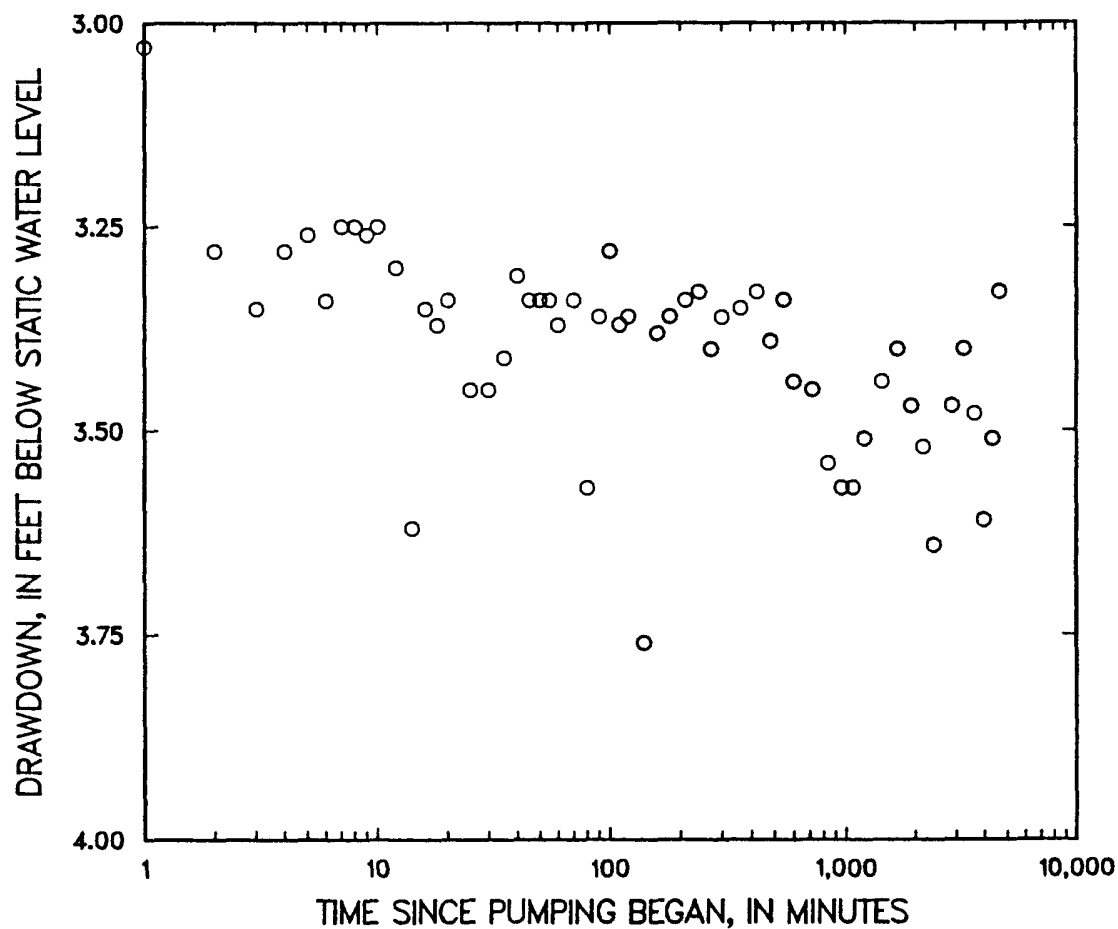

FIGURE 14.--Drawdown versus time at MX test well CE-DT-4, December 20-23, 1980. 
TABLE 7.--Water levels during and after a 77-hour, constant-discharge ayuifer test at MX test well $\mathrm{CE}^{\prime}-D^{\prime} T^{\prime}-4$, Necember 20-23, 1980.1 static water level is 352.85 feet below land surface

[TIme, minutes since pumping began; water level, depth below land surface; drawdown, calculated drawdown of water levels in feet below static water leve1; resldual drawdown, calculated drawdown of water levels remaining below static water level]

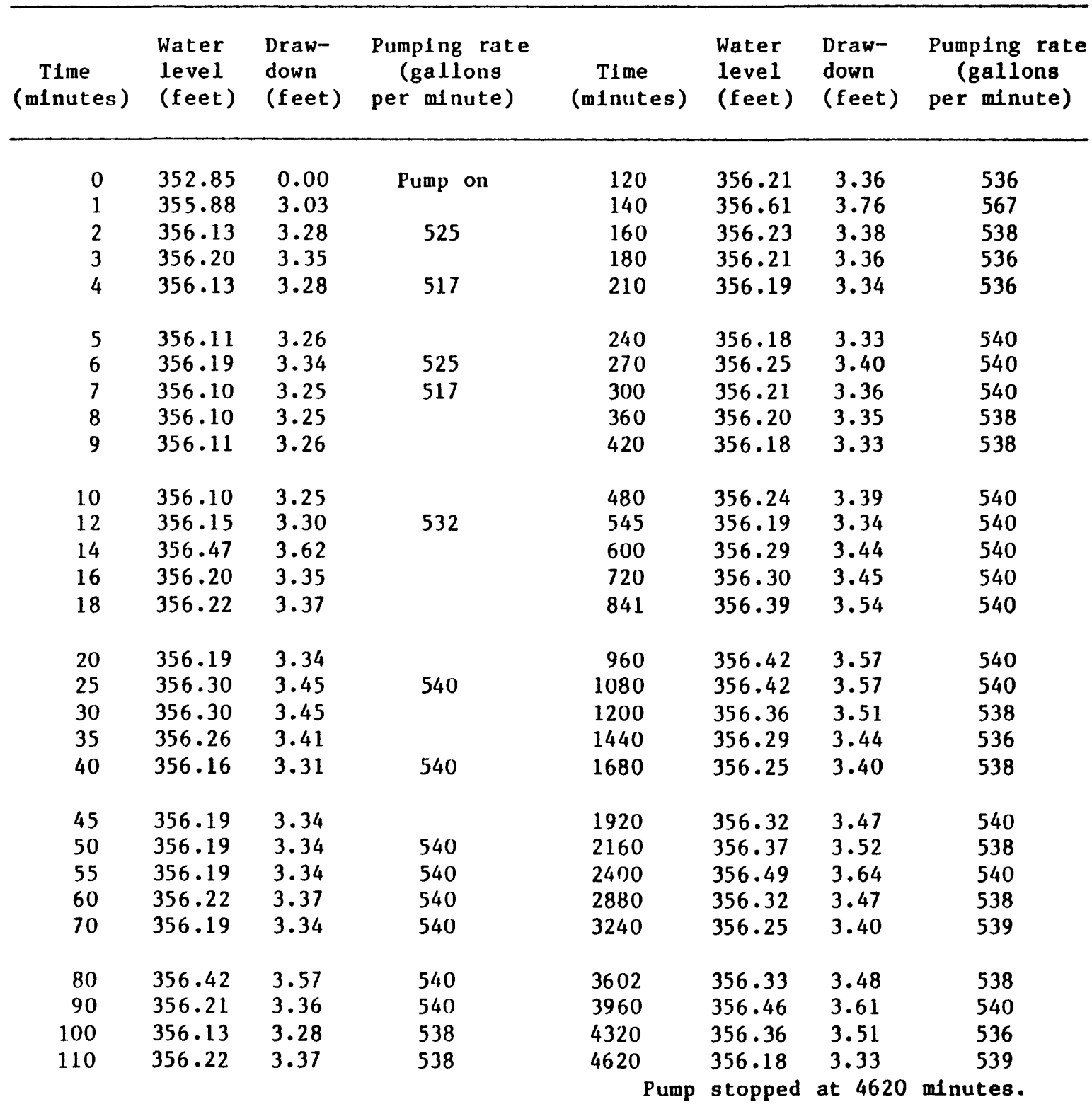


TABLE 7.--Water levels during and after a 77-hour, constantdischarge aquifer test at MX test well C'E-DT-4, Necember $20-23,1980^{1--}$ Continued

\begin{tabular}{lccccc}
\hline $\begin{array}{c}\text { Time } \\
\text { (minutes) }\end{array}$ & $\begin{array}{c}\text { Water leve1 } \\
\text { (feet) }\end{array}$ & $\begin{array}{c}\text { Residual } \\
\text { drawdown } \\
\text { (feet) }\end{array}$ & $\begin{array}{c}\text { Time } \\
\text { (minutes) }\end{array}$ & $\begin{array}{c}\text { Water leve1 } \\
\text { (feet) }\end{array}$ & $\begin{array}{c}\text { Res1dual } \\
\text { drawdown } \\
\text { (feet) }\end{array}$ \\
\hline 4621 & 353.15 & 0.30 & 4626 & 352.80 & -0.05 \\
4621.5 & 352.88 & 0.03 & 4627 & 352.83 & -0.02 \\
4622 & 352.85 & 0.00 & 4628 & 352.84 & -0.01 \\
4622.5 & 352.82 & -0.03 & 4629 & 352.87 & 0.02 \\
4623.25 & 352.82 & -0.03 & 4630 & 352.87 & 0.02 \\
4624 & 352.83 & -0.02 & 4635 & 352.87 & 0.02 \\
4625 & 352.84 & -0.01 & 4640 & 352.87 & 0.02 \\
\hline
\end{tabular}

1 From Ertec Western, Inc., written communication, 1981. 


\section{CE-DT-5}

See section on well CE-DT-4 for location of this well. Drilling began on CE-DT-5 on April 13, 1981. It was completed on May 6, 1981, at a total depth of 628 feet. Limestone bedrock was reported at 110 feet and the water table was reached at 350 feet below land surface. The well was cased with 20-inch-diameter steel casing and cemented to 126 feet. Frequent loss of circulation of drilling fluid was recorded. Two zones of significant circulation loss were encountered at 387 feet and 571 feet. The upper zone was cemented off and drilling continued through the lower zone until it became apparent that significant saturated fractures were penetrated. The drilling penetration rate and 1 ithologic $\log$ for this well are show in figure 15 . A temperature $\log$ is shown in figure 16 .

We11 CE-DT-5 was tested during August and September of 1981. The well was developed by bailing 37 times. The bailer held 30.8 gallons. A vertical turbine pump with 10-inch-diameter discharge pipe was used in testing the we11. The intake was set at 500 feet. Discharge was measured with orifice plates and a piezometer tube, and was piped 200 feet to an alluvial channel. Water levels were measured in the we11 and at observation well CE-DT-4 with a recording pressure transducer. Periodic checks were made by electric sounder and barometer to correct for atmospheric-induced drift. Water levels at six other observation wells and discharge at six springs were monitored daily but no effects due to pumping were discerned. The test, which was the eighth in a series of constant and step discharge tests, lasted 30 days and 3 hours, with a 3-hour shutdown for maintenance 13 days and 14 hours into the test. The initial increase in drawdown between 0.07 and 0.60 minutes, as shown in figure 17 , represents the filling of the pump column before water from the formation was pumped. Table 8 and figure 17 present data from $\mathrm{MX}$ test well $\mathrm{CE}-\mathrm{DT}-5$, and table 9 and figure 18 present data from observation well CE-DT-4 during the testing at CE-DT-5. 


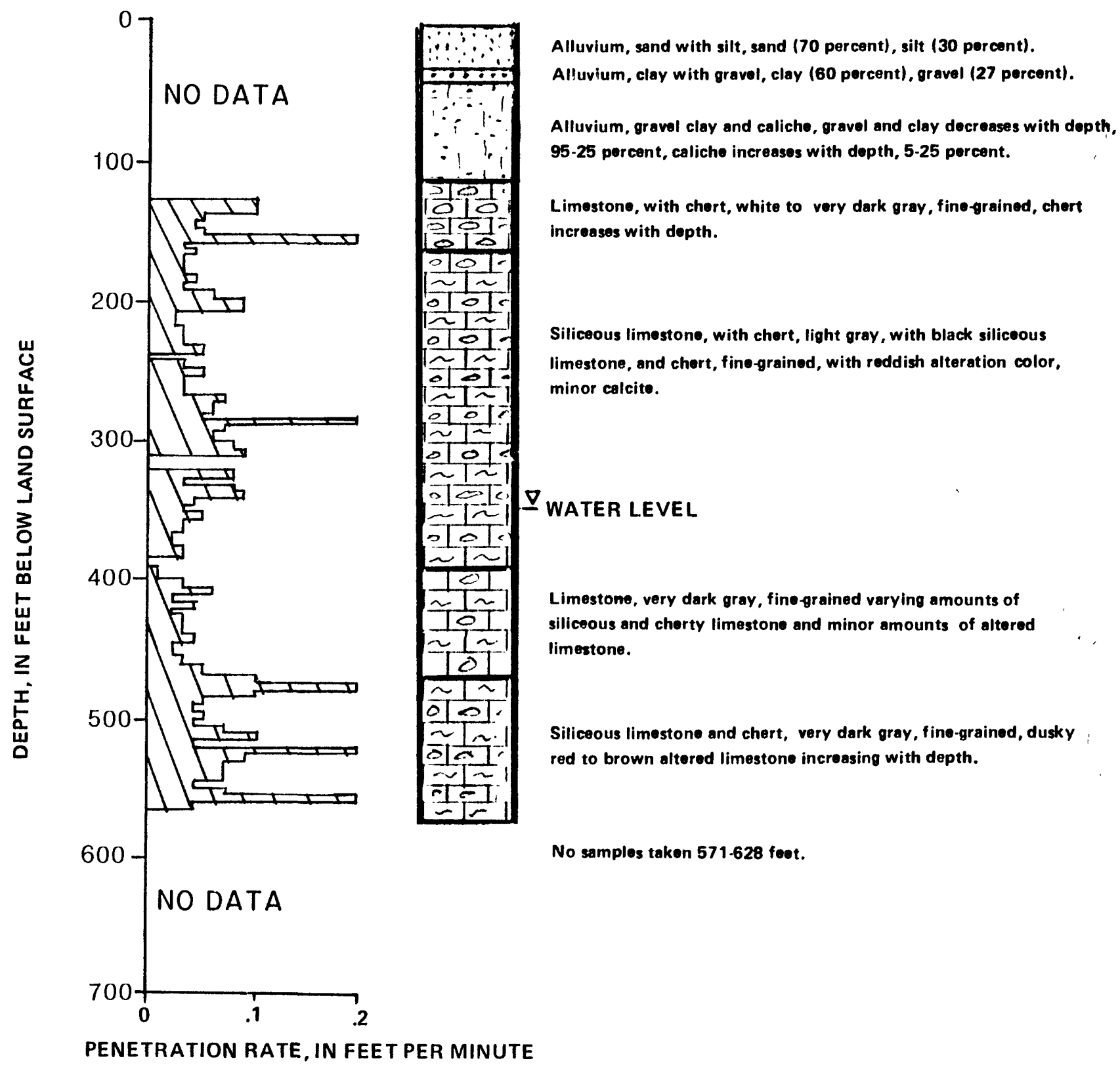

FIGURE 15.--Drilling penetration rate and lithology for $M X$ test well CE-DT-5. 


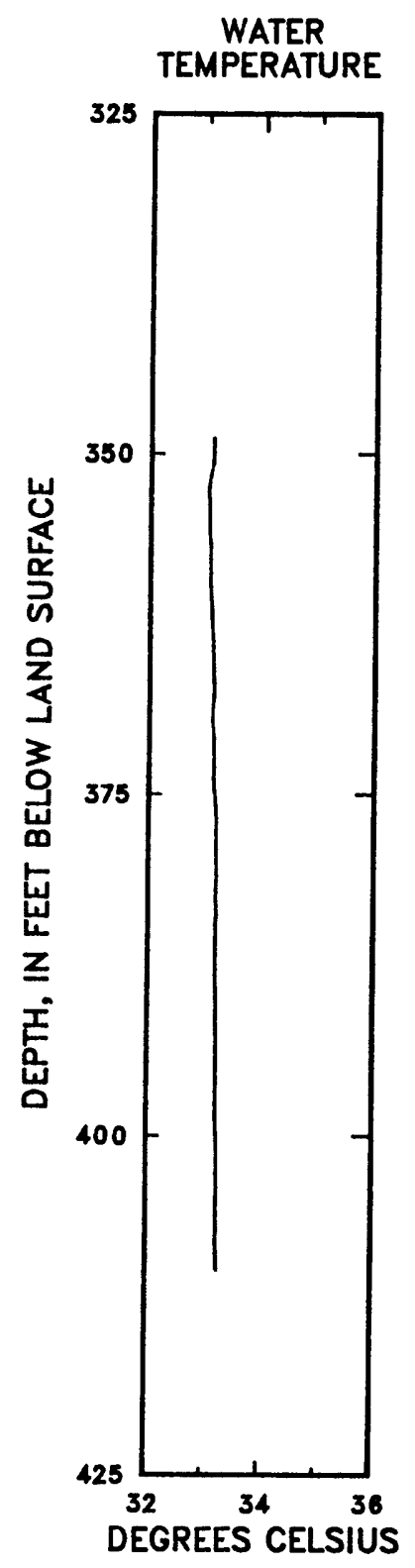

FIGURE 16.--Water-temperature log for MX test well CE-DT-5. 


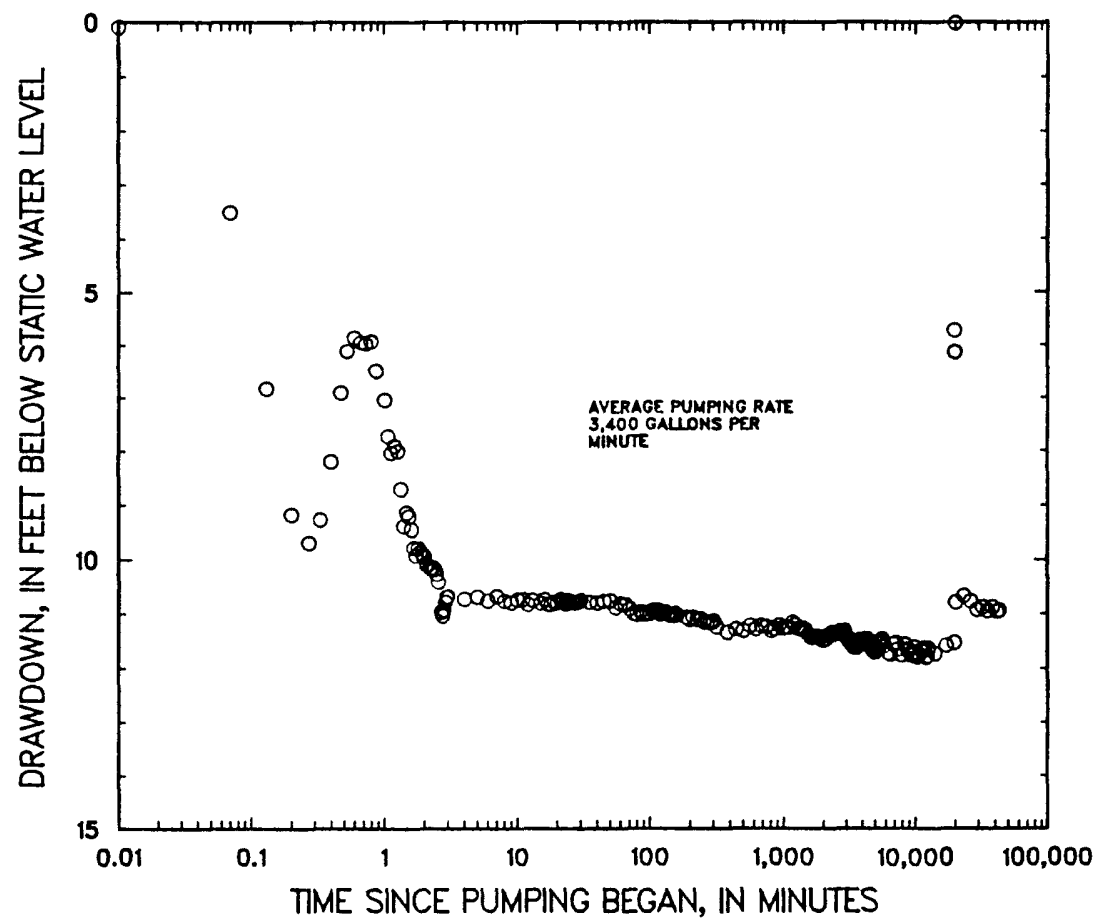

FIGURE 17.--Drawdown versus time at pumped $M X$ test well CE.DT.5, August 28 to September 27, 1981.

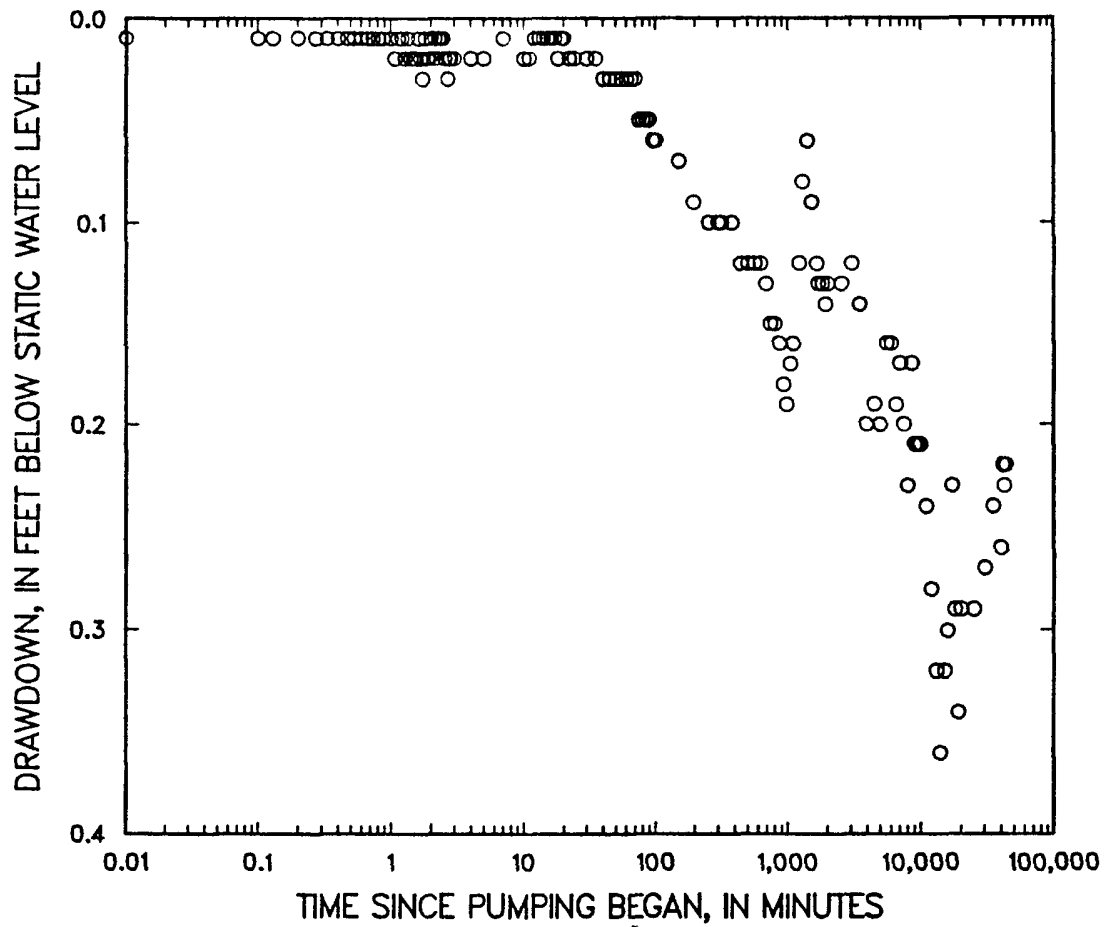

FIGURE 18.--Drawdown versus time at MX test well CE-DT-4, during pumping at $M X$ test well CE-DT-5, August 28 to September 27, 1981. 
TABLE 8.--Water levels during a 30-day, constant-discharge ayuifer test at $M X$ test well $C E^{\prime}-D T^{\prime}-5$, August 28 to september 28 , 1981.1 Static water level $i s$ 349.5. Pumping rate was reported to be constant at 3,400 gallons per minute

[Time, minutes since pumping began; water leve1, depth in feet below land surface; drawdown, calculated drawdown of water levels below static water leve1]

\begin{tabular}{|c|c|c|c|c|c|}
\hline $\begin{array}{c}\text { Time } \\
\text { (minutes) }\end{array}$ & $\begin{array}{l}\text { Water } \\
\text { leve } 1 \\
\text { (feet) }\end{array}$ & $\begin{array}{c}\text { Drawdown } \\
\text { (feet) }\end{array}$ & $\begin{array}{c}\text { Time } \\
\text { (minutes) }\end{array}$ & $\begin{array}{l}\text { Water } \\
\text { leve } 1 \\
\text { (feet) }\end{array}$ & $\begin{array}{c}\text { Drawdown } \\
\text { (feet) }\end{array}$ \\
\hline 0.0 & 349.50 & 0.00 (pump on) & 2.0 & 359.44 & 9.94 \\
\hline 0.0 & 349.60 & 0.10 & 2.1 & 359.61 & 10.11 \\
\hline 0.1 & 353.01 & 3.51 & 2.1 & 359.61 & 10.11 \\
\hline 0.1 & 356.33 & 6.83 & 2.2 & 359.67 & 10.17 \\
\hline 0.2 & 358.67 & 9.17 & 2.3 & 359.68 & 10.18 \\
\hline 0.3 & 359.19 & 9.69 & 2.3 & 359.65 & 10.15 \\
\hline 0.3 & 358.77 & 9.27 & 2.4 & 359.69 & 10.19 \\
\hline 0.4 & 357.70 & 8.20 & 2.5 & 359.77 & 10.27 \\
\hline 0.5 & 356.41 & 6.91 & 2.5 & 359.91 & 10.41 \\
\hline 0.5 & 355.62 & 6.12 & 2.7 & 360.47 & 10.97 \\
\hline 0.6 & 355.36 & 5.86 & 2.7 & 360.54 & 11.04 \\
\hline 0.7 & 355.45 & 5.95 & 2.8 & 360.43 & 10.93 \\
\hline 0.7 & 355.48 & 5.98 & 2.9 & 360.29 & 10.79 \\
\hline 0.8 & 355.43 & 5.93 & 2.9 & 360.19 & 10.69 \\
\hline 0.9 & 355.99 & 6.49 & 3.0 & 360.19 & 10.69 \\
\hline 1.0 & 356.55 & 7.05 & 4.0 & 360.23 & 10.73 \\
\hline 1.1 & 357.23 & 7.73 & 5.0 & 360.19 & 10.69 \\
\hline 1.1 & 357.54 & 8.04 & 6.0 & 360.26 & 10.76 \\
\hline 1.2 & 357.41 & 7.91 & 7.0 & 360.18 & 10.68 \\
\hline 1.3 & 357.51 & 8.01 & 8.0 & 360.27 & 10.77 \\
\hline 1.3 & 358.21 & 8.71 & 9.0 & $360 \cdot 30$ & 10.80 \\
\hline 1.4 & 358.89 & 9.39 & 10.0 & 360.24 & 10.74 \\
\hline 1.5 & 358.64 & 9.14 & 11.0 & 360.24 & 10.74 \\
\hline 1.5 & 358.71 & 9.21 & 12.0 & 360.33 & 10.83 \\
\hline 1.6 & 358.95 & 9.45 & 13.0 & 360.25 & 10.75 \\
\hline 1.7 & 359.29 & 9.79 & 15.0 & 360.31 & 10.81 \\
\hline 1.7 & 359.43 & 9.93 & 16.0 & 360.24 & 10.74 \\
\hline 1.8 & 359.30 & 9.80 & 17.0 & 360.33 & 10.83 \\
\hline 1.9 & 359.36 & 9.86 & 18.0 & 360.33 & 10.83 \\
\hline 1.9 & 359.42 & 9.92 & 19.0 & 360.29 & 10.79 \\
\hline
\end{tabular}


TABLE 8.--Water levels during a 30-day, constant-discharge aquifer test at $M X$ well $C E^{\prime}-D I^{\prime}-5$, August 28 to September 28, 19811--Continued

\begin{tabular}{|c|c|c|c|c|c|c|}
\hline $\begin{array}{c}\text { Time } \\
\text { (minutes) }\end{array}$ & $\begin{array}{l}\text { Water } \\
\text { leve } 1 \\
\text { (feet) }\end{array}$ & $\begin{array}{c}\text { Drawdown } \\
\text { (feet) }\end{array}$ & $\begin{array}{c}\text { Time } \\
\text { (minutes) }\end{array}$ & $\begin{array}{l}\text { Water } \\
\text { leve } 1 \\
\text { (feet) }\end{array}$ & $\begin{array}{c}\text { Drawdown } \\
\text { (feet) }\end{array}$ & \\
\hline 20.0 & $360 \cdot 31$ & 10.81 & 155.0 & 360.50 & 11.00 & \\
\hline 21.0 & 360.24 & 10.74 & 160.0 & 360.53 & 11.03 & \\
\hline 22.0 & 360.29 & 10.79 & 186.0 & 360.57 & 11.07 & \\
\hline 23.0 & 360.33 & 10.83 & 196.0 & 360.61 & 11.11 & \\
\hline 24.0 & 360.26 & 10.76 & 211.0 & 360.57 & 11.07 & \\
\hline 25.0 & 360.31 & 10.81 & 226.0 & 360.61 & 11.11 & \\
\hline 26.0 & 360.29 & 10.79 & 241.0 & 360.60 & 11.10 & \\
\hline 27.0 & 360.31 & 10.81 & 256.0 & 360.66 & 11.16 & \\
\hline 28.0 & 360.29 & 10.79 & 271.0 & 360.66 & 11.16 & \\
\hline 29.0 & 360.29 & 10.79 & 286.0 & 360.66 & 11.16 & \\
\hline 30.0 & 360.26 & 10.76 & 301.0 & 360.64 & 11.14 & . \\
\hline 35.0 & 360.29 & 10.79 & 316.0 & 360.74 & 11.24 & \\
\hline 40.0 & 360.30 & 10.80 & 381.0 & 360.84 & 11.34 & \\
\hline 45.0 & 360.27 & 10.77 & 441.0 & 360.78 & 11.28 & \\
\hline 50.0 & 360.26 & 10.76 & 501.0 & 360.81 & 11.31 & \\
\hline 55.0 & 360.39 & 10.89 & 561.0 & 360.72 & 11.22 & \\
\hline 60.0 & 360.32 & 10.82 & 621.0 & 360.78 & 11.28 & \\
\hline 65.0 & 360.34 & 10.84 & 681.0 & 360.72 & 11.22 & \\
\hline 70.0 & 360.41 & 10.91 & 741.0 & 360.74 & 11.24 & \\
\hline 75.0 & 360.50 & 11.00 & 801.0 & 360.81 & 11.31 & \\
\hline 80.0 & 360.52 & 11.02 & 861.0 & 360.78 & 11.28 & \\
\hline 85.0 & 360.47 & 10.97 & 921.0 & 360.71 & 11.21 & \\
\hline $\begin{array}{l}90.0 \\
95.0\end{array}$ & $\begin{array}{l}360.52 \\
360.48\end{array}$ & $\begin{array}{l}11.02 \\
10.98\end{array}$ & $\begin{array}{r}981.0 \\
1041.0\end{array}$ & $\begin{array}{l}360.77 \\
360.75\end{array}$ & $\begin{array}{l}11.27 \\
11.25\end{array}$ & \\
\hline 100.0 & 360.51 & 11.01 & 1101.0 & 360.75 & 11.25 & \\
\hline 105.0 & 360.44 & 10.94 & 1161.0 & 360.66 & 11.16 & \\
\hline 110.0 & 360.48 & 10.98 & 1221.0 & 360.70 & 11.20 & \\
\hline 115.0 & 360.44 & 10.94 & 1281.0 & 360.78 & 11.28 & \\
\hline 120.0 & 360.52 & 11.02 & 1341.0 & 360.79 & 11.29 & \\
\hline 125.0 & 360.50 & 11.00 & 1401.0 & 360.77 & 11.27 & \\
\hline 130.0 & 360.47 & 10.97 & 1461.0 & 360.80 & 11.30 & \\
\hline 135.0 & 360.52 & 11.02 & 1521.0 & 360.89 & 11.39 & \\
\hline 140.0 & 360.54 & 11.04 & 1581.0 & 360.93 & 11.43 & \\
\hline 145.0 & 360.52 & 11.02 & 1641.0 & 360.94 & 11.44 & \\
\hline 150.0 & 360.54 & 11.04 & 1701.0 & 360.93 & 11.43 & \\
\hline
\end{tabular}


TABLE 8.--Water levels during a 30-day, constant-discharge aquifer test at MX well CE'DT'5, August 28 to September 28, 19811--Cont1nued

\begin{tabular}{|c|c|c|c|c|c|}
\hline $\begin{array}{c}\text { Time } \\
\text { (minutes) }\end{array}$ & $\begin{array}{l}\text { Water } \\
\text { leve } 1 \\
\text { (feet) }\end{array}$ & $\begin{array}{c}\text { Drawdown } \\
\text { (feet) }\end{array}$ & $\begin{array}{c}\text { Time } \\
\text { (minutes) }\end{array}$ & $\begin{array}{l}\text { Water } \\
\text { leve } 1 \\
\text { (f eet) }\end{array}$ & $\begin{array}{c}\text { Drawdown } \\
\text { (feet) }\end{array}$ \\
\hline 1761.0 & 360.91 & 11.41 & 3861.0 & 361.05 & 11.55 \\
\hline 1821.0 & 360.91 & 11.41 & 3921.0 & 361.07 & 11.57 \\
\hline 1881.0 & 360.94 & 11.44 & 3981.0 & 361.00 & 11.50 \\
\hline 1941.0 & 360.99 & 11.49 & 4041.0 & 361.02 & 11.52 \\
\hline 2001.0 & 360.99 & 11.49 & 4101.0 & 360.99 & 11.49 \\
\hline 2061.0 & 360.96 & 11.46 & 4161.0 & 360.96 & 11.46 \\
\hline 2121.0 & 360.96 & 11.46 & 4221.0 & 361.07 & 11.57 \\
\hline 2181.0 & 360.88 & 11.38 & 4281.0 & 361.02 & 11.52 \\
\hline 2241.0 & 360.88 & 11.38 & 4341.0 & 360.96 & 11.46 \\
\hline 2301.0 & 360.84 & 11.34 & 4401.0 & 361.05 & 11.55 \\
\hline 2361.0 & 360.86 & 11.36 & 4461.0 & 361.05 & 11.55 \\
\hline 2431.0 & 360.85 & 11.35 & 4521.0 & 361.00 & 11.50 \\
\hline 2481.0 & 360.88 & 11.38 & 4581.0 & 361.09 & 11.59 \\
\hline 2541.0 & 360.87 & 11.37 & 4641.0 & $361 \cdot 10$ & 11.60 \\
\hline 2601.0 & 360.82 & 11.32 & 4701.0 & 361.12 & 11.62 \\
\hline 2661.0 & 360.88 & 11.38 & 4761.0 & 361.19 & 11.69 \\
\hline 2721.0 & 360.89 & 11.39 & 4821.0 & 361.19 & 11.69 \\
\hline 2781.0 & 360.84 & 11.34 & 4881.0 & 361.21 & 11.71 \\
\hline 2841.0 & 360.88 & 11.38 & 4941.0 & 361.18 & 11.68 \\
\hline 2901.0 & 360.81 & 11.31 & 5001.0 & 361.20 & 11.70 \\
\hline 2961.0 & 360.93 & 11.43 & 5061.0 & 361.20 & 11.70 \\
\hline 3021.0 & 360.96 & 11.46 & 5121.0 & 361.12 & 11.62 \\
\hline 3081.0 & 361.02 & 11.52 & 5241.0 & 361.01 & 11.51 \\
\hline 3141.0 & 360.96 & 11.46 & 5301.0 & 361.05 & 11.55 \\
\hline 3201.0 & 361.00 & 11.50 & 5421.0 & 361.03 & 11.53 \\
\hline $326 \mathrm{l} .0$ & 361.09 & 11.59 & 5541.0 & 361.01 & 11.51 \\
\hline 3321.0 & 361.07 & 11.57 & 5601.0 & 360.96 & 11.46 \\
\hline 3381.0 & 361.12 & 11.62 & 5721.0 & 361.03 & 11.53 \\
\hline 3441.0 & 361.05 & 11.55 & 4841.0 & 361.08 & 11.58 \\
\hline 3501.0 & 361.09 & 11.59 & 5901.0 & 361.09 & 11.59 \\
\hline 3561.0 & 361.05 & 11.55 & 6201.0 & 361.22 & 11.72 \\
\hline 3621.0 & 361.12 & 11.62 & 6501.0 & 361.25 & 11.75 \\
\hline 3681.0 & 361.00 & 11.50 & 6801.0 & 361.06 & 11.56 \\
\hline 3741.0 & 360.98 & 11.48 & 7101.0 & 361.03 & 11.53 \\
\hline 3801.0 & 361.01 & 11.51 & 7401.0 & 361.15 & 11.65 \\
\hline
\end{tabular}


TABLE 8.--Water levels during a 30-day, constant-discharge aquifer test at $M X$ well CE-DT-5, August 28 to September 28, 1.981--Continued

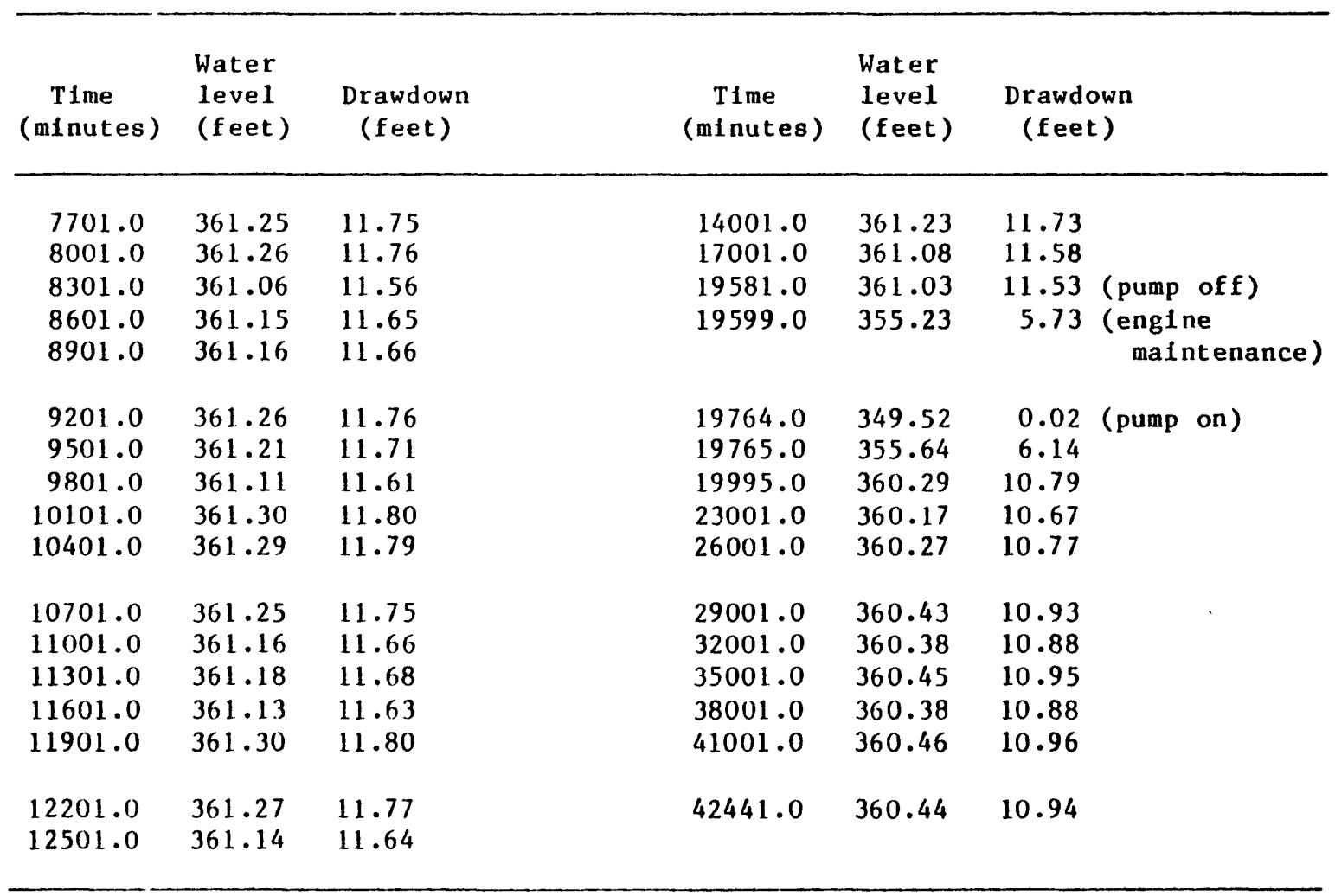

1 From Ertec Western, Inc., 1981. 
TABI.E 9.--Water levels at observation test well CE'-VI'-4 during a 30-day, constant-discharge aquif'er test at test well CE'-VT-5, August 28 to September 27, 1981.1 Pumping rate at well CE-UT-5 reported to be 3,400 gallons per minute. Static water level is 352.30 feet below land surface

[Time, minutes since pumping began; water level, depth below land surface; drawdown, calculated drawdown of water levels below static water leve1; residual drawdown, calculated drawdown of water levels remalning below static water level]

\begin{tabular}{|c|c|c|c|c|c|}
\hline $\begin{array}{c}\text { Tíme } \\
\text { (minutes) }\end{array}$ & $\begin{array}{l}\text { Water } \\
\text { leve } 1 \\
\text { (feet) }\end{array}$ & $\begin{array}{c}\text { Drawdown } \\
(\text { feet })\end{array}$ & $\begin{array}{c}\text { Time } \\
\text { (m1 nutes) }\end{array}$ & $\begin{array}{l}\text { Water } \\
\text { leve1 } \\
\text { (feet) }\end{array}$ & $\begin{array}{l}\text { Drawdown } \\
\text { (f eet })\end{array}$ \\
\hline 0.00 & 352.30 & 0.00 (pump on) & 1.93 & 352.31 & 0.01 \\
\hline 0.07 & 352.30 & 0.00 & 2.00 & 352.31 & 0.01 \\
\hline 0.13 & 352.30 & 0.00 & 2.07 & 352.32 & 0.02 \\
\hline 0.20 & 352.30 & 0.00 & 2.13 & 352.31 & 0.01 \\
\hline 0.27 & 352.31 & 0.01 & 2.20 & 352.32 & 0.02 \\
\hline 0.33 & 352.31 & 0.01 & 2.27 & 352.31 & 0.01 \\
\hline 0.40 & 352.30 & 0.00 & 3.00 & 352.32 & 0.02 \\
\hline 0.47 & 352.31 & 0.01 & 10.0 & 352.32 & 0.02 \\
\hline 0.53 & 352.32 & 0.00 & 20.0 & 352.31 & 0.01 \\
\hline 0.60 & 352.30 & 0.00 & 30.0 & 352.32 & 0.02 \\
\hline 0.67 & 352.31 & 0.01 & 100.0 & 352.32 & 0.02 \\
\hline 0.73 & 352.31 & 0.01 & 196.0 & 352.39 & 0.09 \\
\hline 0.80 & 352.31 & 0.01 & 301.0 & 352.40 & 0.10 \\
\hline 0.87 & 352.31 & 0.01 & 981.0 & 352.49 & 0.19 \\
\hline 0.93 & 352.31 & 0.01 & 2001.0 & 352.43 & 0.13 \\
\hline 1.00 & 352.31 & 0.01 & 3021.0 & 352.42 & 0.12 \\
\hline 1.07 & 352.32 & 0.02 & 3981.0 & 352.50 & 0.20 \\
\hline 1.13 & 352.31 & 0.01 & 5001.0 & 352.50 & 0.20 \\
\hline 1.20 & 352.30 & 0.00 & 5901.0 & 352.44 & 0.14 \\
\hline 1.27 & 352.32 & 0.02 & 7101.0 & 352.45 & 0.15 \\
\hline 1.33 & 352.31 & 0.01 & 8001.0 & 352.53 & 0.23 \\
\hline 1.40 & 352.32 & 0.02 & 8961.0 & 352.50 & 0.20 \\
\hline 1.47 & 352.32 & 0.02 & 10101.0 & 352.54 & 0.24 \\
\hline 1.53 & $352 \cdot 32$ & 0.02 & 11001.0 & 352.54 & 0.24 \\
\hline 1.60 & 352.31 & 0.01 & 12021.0 & 352.58 & 0.28 \\
\hline 1.67 & 352.32 & 0.02 & 14001.0 & 352.66 & 0.36 \\
\hline 1.73 & 352.33 & 0.03 & 17061.0 & 352.53 & 0.23 \\
\hline 1.80 & 352.31 & 0.01 & 19599.0 & 352.63 & 0.33 (pump of $f$ ) \\
\hline 1.87 & 352.32 & 0.02 & & & \\
\hline
\end{tabular}


TABI.E 9.--Water levels at observation test well CE-UT-4, during a 30-day, constant-discharge aquifer test at test well Ct'-VT-5, August 28 to September $27,1981^{1--C o n t}$ Inued

\begin{tabular}{lccccc}
$\begin{array}{c}\text { Tine } \\
\text { (minutes) }\end{array}$ & $\begin{array}{c}\text { Water 1eve1 } \\
\text { (feet) }\end{array}$ & $\begin{array}{c}\text { Residual } \\
\text { drawdown } \\
\text { (feet) }\end{array}$ & $\begin{array}{c}\text { Time } \\
\text { (minutes) }\end{array}$ & $\begin{array}{c}\text { Water 1evel } \\
\text { (feet) }\end{array}$ & $\begin{array}{c}\text { Residual } \\
\text { drawdown } \\
\text { (feet) }\end{array}$ \\
\hline 23001.0 & 352.56 & 0.26 & 35021.0 & 352.54 & 0.24 \\
26021.0 & 352.63 & 0.33 & 38021.0 & 352.52 & 0.22 \\
29021.0 & 352.59 & 0.29 & 41021.0 & 352.52 & 0.22 \\
32021.0 & 352.52 & 0.22 & & & \\
\hline
\end{tabular}

1 From Ertec Western, Inc., 1981. 


\section{CE-DT-6}

We11 CE-DT-6 is in Moapa Valley, Clark County, adjacent to State Route 168 approximately $6.7 \mathrm{miles}$ east of CE-DT-5 (figure 1). The we11 site is in the southern portion of the Meadow Valley Mountains and was drilled as an observation well for use during aquifer tests at CE-DT-5. Limestone bedrock and the water table were reached at 78 and 457.4 feet below the land surface, respectively.

Drilling began on May 21, 1981, and was completed on June 3, 1981 . A total depth of 937 feet was attained. Eighty-seven feet of 12-3/4inch surface casing was cemented into place and 8-5/8-inch well casing was installed from land surface to the bedrock. The drilling penetration rate and lithologic $\log$ are shown in figure 19. Temperature and caliper logs are shown in figure 20 .

Water levels measured from October 28,1985 , to January 28 , 1986, for this well are presented in graphic form in figure 21 . The Geological Survey made a 66-hour constant-discharge aquifer test December 6-12, 1986. Drawdown data are listed in table 10 and shown in figure 22. Recovery data are listed in table 11 and shown in figure 23. Water levels were measured with a recording pressure transducer calibrated onsite. Total recovery occurred within 5 minutes after the pump was turned of $f$. 


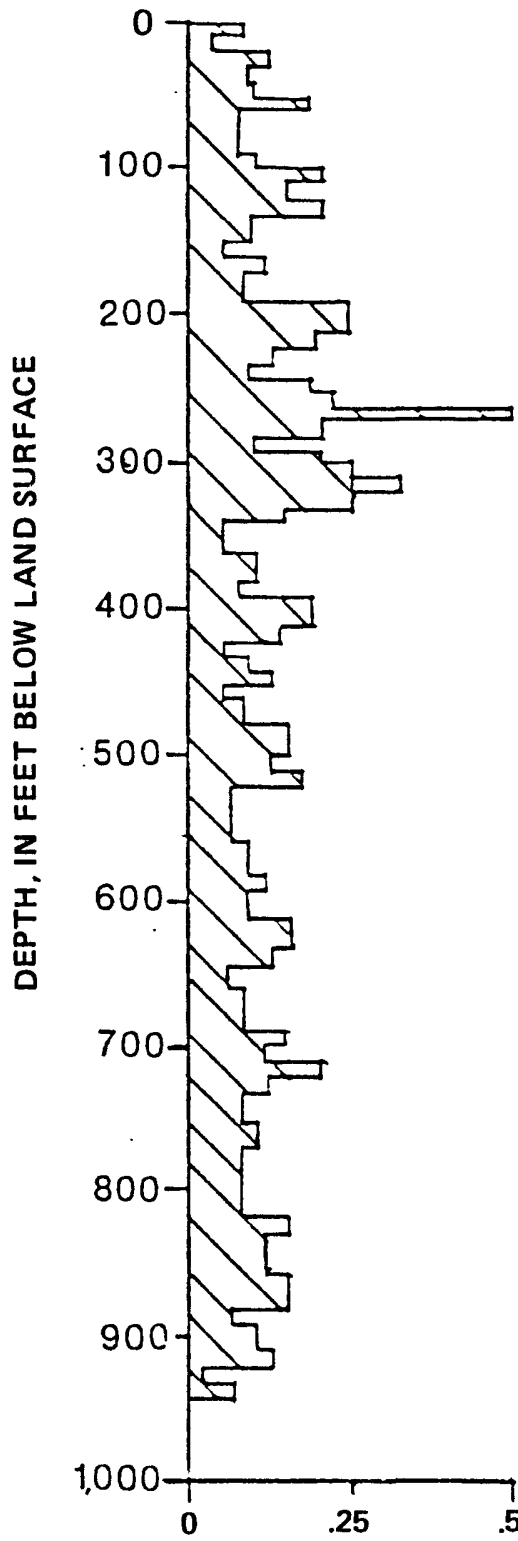

Conglometate, caliche cemented, with gravels of gray limestone
siliceous limestone, black chert, and volcanic fragments.

-0.0

का

ole

10

il i-

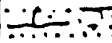

$\because \frac{\ldots . \cdots}{\because \cdots}$

$\because \frac{1}{\because 19}$

בc: 12

$x-x$

$2-2$

\begin{tabular}{|c|c|}
\hline 010 \\
\hline 1010
\end{tabular}

$\Leftrightarrow 12$

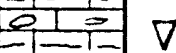

010

$-1-1-$

010

- 10

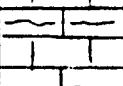

- -

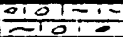

$-1-1-$

का

-15 -

010

\begin{tabular}{c|c}
$-\mathrm{E}$ & $\mathrm{C}$ \\
\hline $\mathrm{C}$ & 0
\end{tabular}

$-1-1-$

0 o

$-1-1-$

0 0

$-1-1-$

010

$-1-1-$

Limestone, siliceous gray to very dark gray, black chert with varying amounts of altered limastone, calcite vein material,

and reddish calcareous clay.

Sandstone, light gray, well sorted, with calcareous pink ish clay.

Rhyolite, reddish porphyry tic quartz rhyolite in red aphanitic matrix.

Limestone, light to very dark siliceous, black chert, varying amounts of calcite and calcareous clay.

\section{$\nabla$}

WATER LEVEL

Limestone, very light gray to white, with varying amounts of calcareous clay.

Limestone, light gray to white, with increasing amounts of siliceous limestone chert and calcite.

Limestone, light to very dark gray siliceous, with chert and varying amounts of calcareous clay.

FIGURE 19.--Drilling penetration rate and lithology for $M X$ test well CE-DT-6. 

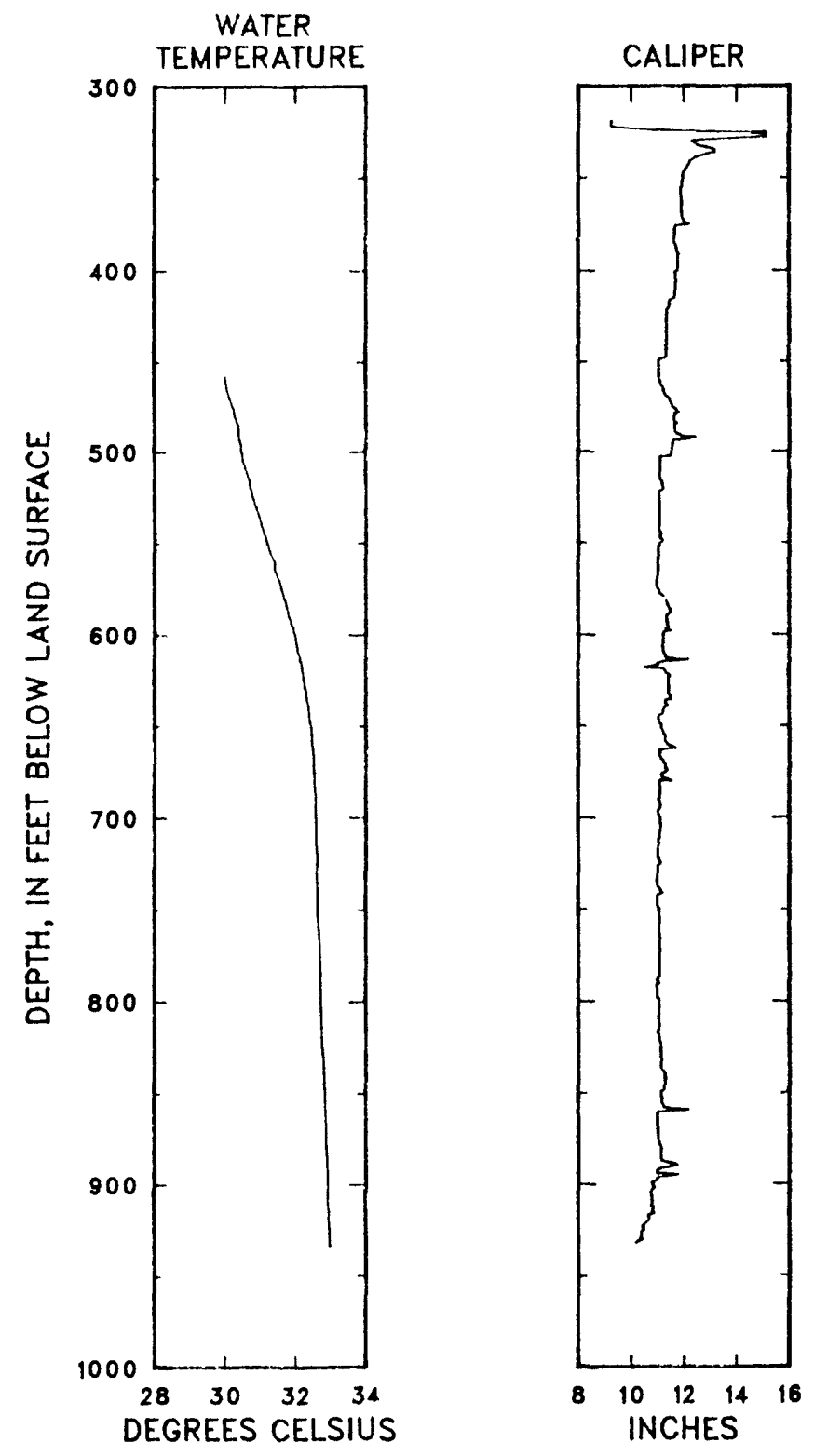

FIGURE 20.--Water temperature and caliper logs for $M X$ test well CE-DT-6. 


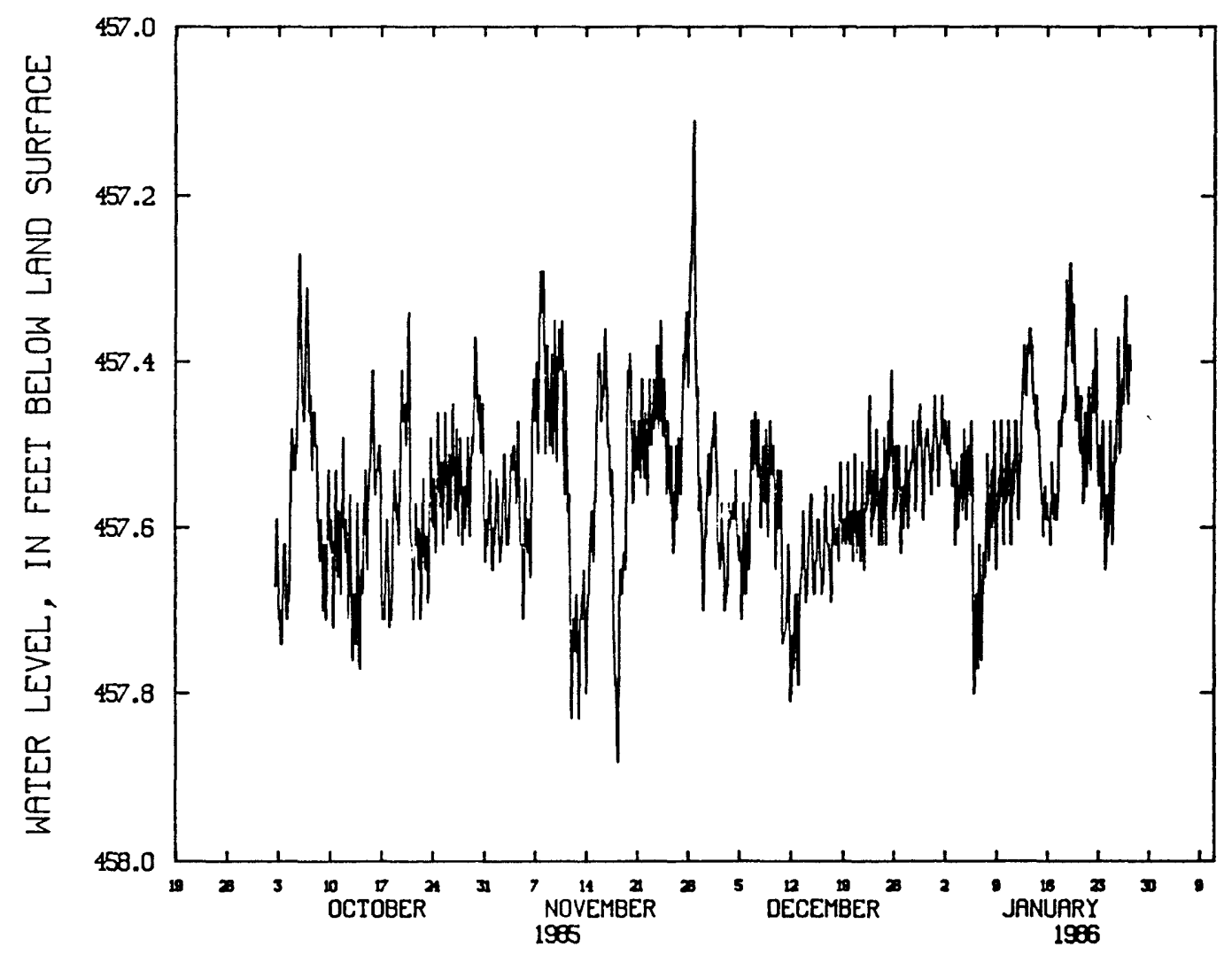

FIGURE 21.--Data from continuous water-level recording transducer at MX test well CE-DT-6 for October 28, 1985, to January 28, 1986. 


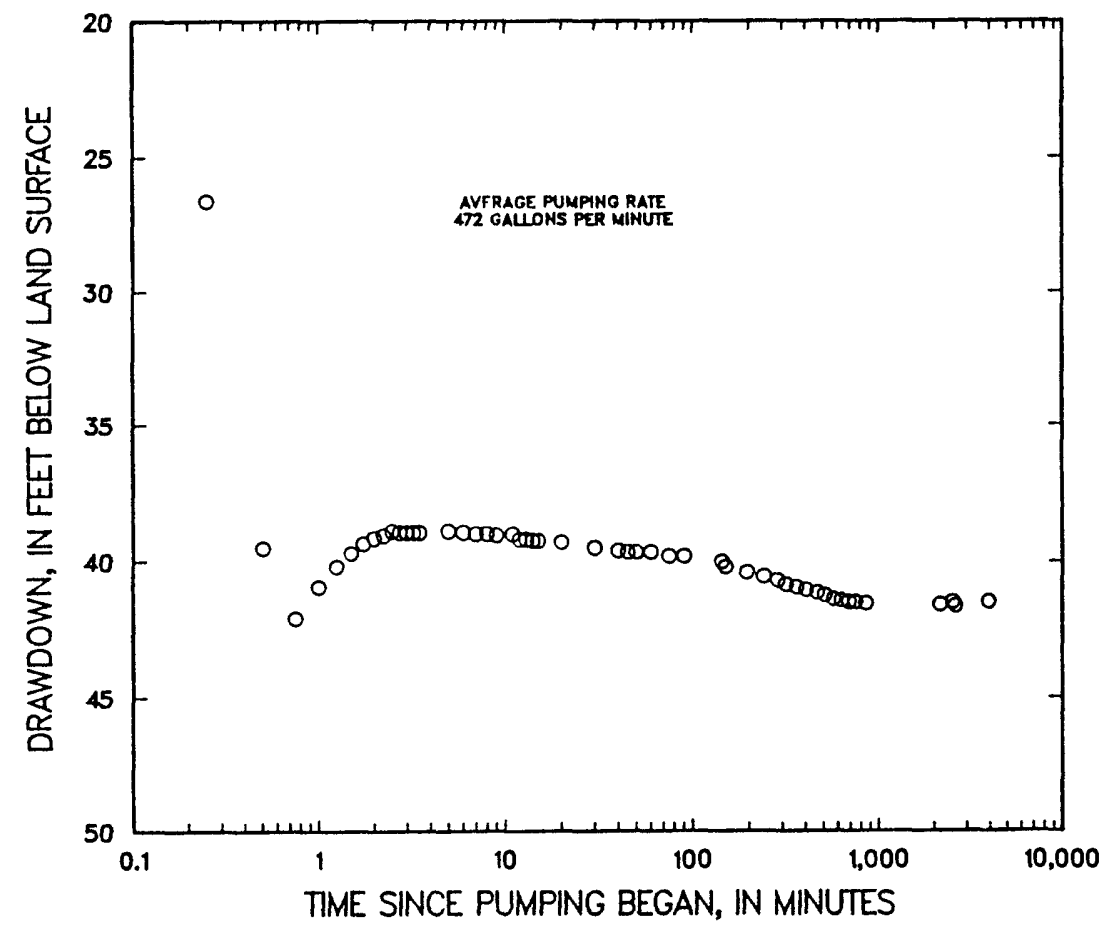

FIGURE 22.--Drawdown versus time at MX test well CE-DT-6, December 9-12, 1986.

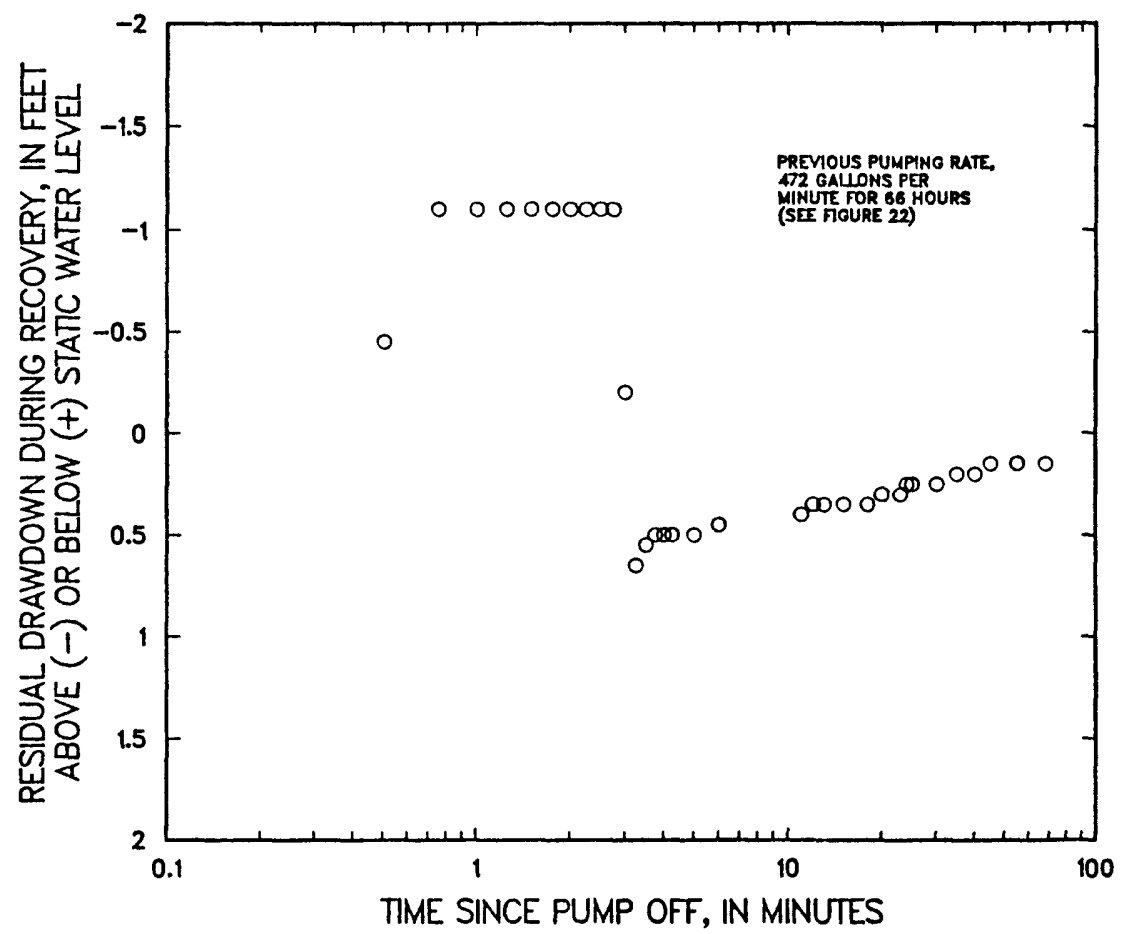

FIGURE 23.--Residual drawdown versus time at MX test well CE-DT-6, December 12, 1986. 
TABLE 10.--Water levels during a 66-hour, constant-discharge aquifer test at $M X$ test well $C E^{\prime}-U T-6$, December 9-12, 1986. Static water level is 457.3 feet

[TIme, minutes since pumping began; water level, depth below land surface; drawdown, calculated drawdown of water levels in feet below static water level; pumping rate, spot-checked during test (average pumping rate during entire test determined from flow totalizer was 472.0 gallons per minute)]

\begin{tabular}{|c|c|c|c|c|c|c|c|}
\hline $\begin{array}{c}\text { T1me } \\
\text { (minutes) }\end{array}$ & $\begin{array}{l}\text { Water } \\
\text { leve1 } \\
\text { (feet) }\end{array}$ & $\begin{array}{l}\text { Draw- } \\
\text { down } \\
\text { (feet) }\end{array}$ & $\begin{array}{l}\text { Puinping rate } \\
\text { (gallons } \\
\text { per minute) } 1\end{array}$ & $\begin{array}{c}\text { Time } \\
\text { (minutes) }\end{array}$ & $\begin{array}{l}\text { Water } \\
\text { level } \\
\text { (feet) }\end{array}$ & $\begin{array}{l}\text { Draw- } \\
\text { down } \\
\text { (feet) }\end{array}$ & $\begin{array}{l}\text { Pumping rate } \\
\quad \text { (gallons } \\
\text { per minute) }\end{array}$ \\
\hline 0.00 & 457.30 & 0.00 & & 30.00 & 496.80 & 39.50 & 462 \\
\hline 0.25 & 483.95 & 26.65 & & 40.00 & 496.90 & 39.60 & \\
\hline 0.50 & 496.80 & 39.50 & & 45.00 & 496.95 & 39.65 & 500 \\
\hline 0.75 & 499.40 & 42.10 & & 50.00 & 496.95 & 39.65 & \\
\hline 1.00 & 498.25 & 40.95 & & 60.00 & 496.95 & 39.65 & 450 \\
\hline 1.25 & 497.50 & 40.20 & & 75.00 & 497.10 & 39.80 & \\
\hline 1.50 & 497.00 & 39.70 & & 90.00 & 497.10 & 39.80 & 466 \\
\hline 1.75 & 496.65 & 39.35 & & 113.00 & 497.30 & 40.00 & 478 \\
\hline 2.00 & 496.45 & 39.15 & & 150.00 & 497.50 & 40.20 & \\
\hline 2.25 & 496.35 & 39.05 & & 195.00 & 497.70 & 40.40 & \\
\hline 2.50 & 496.20 & 38.90 & & 240.00 & 497.85 & 40.55 & \\
\hline 2.75 & 496.25 & 38.95 & & 285.00 & 498.00 & 40.70 & \\
\hline 3.00 & 496.25 & 38.95 & & 315.00 & 498.15 & 40.85 & \\
\hline 3.25 & 496.25 & 38.95 & & 360.00 & 498.25 & 40.95 & \\
\hline 3.50 & 496.25 & 38.95 & & 405.00 & 498.35 & 41.05 & \\
\hline 5.00 & 496.20 & 38.90 & 630 & 465.00 & 498.45 & 41.15 & \\
\hline 6.00 & 496.25 & 38.95 & 480 & 510.00 & 498.55 & 41.25 & \\
\hline 7.00 & 496.30 & 39.00 & & 570.00 & 498.70 & 41.40 & \\
\hline 8.00 & 496.30 & 39.00 & & 630.00 & 498.75 & 41.45 & \\
\hline 9.00 & 496.35 & 39.05 & & 690.00 & 498.80 & 41.50 & \\
\hline 11.00 & 496.30 & 39.00 & & 750.00 & 498.80 & 41.50 & \\
\hline 12.00 & 496.50 & 39.20 & & 855.00 & 498.85 & $41.55^{a}$ & \\
\hline 13.00 & 496.50 & 39.20 & & 2160.00 & 498.90 & 41.60 & \\
\hline 14.00 & 496.55 & 39.25 & & 2520.00 & 498.80 & 41.50 & \\
\hline 15.00 & 496.55 & 39.25 & 428 & 2609.00 & 498.95 & 41.65 & 483 \\
\hline 20.00 & 496.60 & 39.30 & & 3963.00 & 498.80 & 41.50 & 472 \\
\hline
\end{tabular}

1 Th1s well does not have a foot valve; therefore when the pump is first turned on the pumping rate is high until the discharge pipe fills with water up to the surface.

$a$ After 900 minutes, recorded water levels remalned within 0.5 feet of 498.85 feet but fluctuated "randomly" about that level, perhaps due to variations in recorder power supply, barometric pressure, or pumping rate. 
TABLE 11.--Water levels during recovery from a 66-hour, constunt-discharge aquif'er test at MX test well $C E^{\prime}-D T-6$, December 12, 1986

[Tine, minutes since pumping ended; water level, feet below land surface; residual drawdown, calculated drawdown of water levels remalning below static water level]

\begin{tabular}{|c|c|c|c|c|c|}
\hline $\begin{array}{c}\text { Time } \\
\text { (minutes) }\end{array}$ & $\begin{array}{l}\text { Water } \\
\text { leve } 1 \\
\text { (feet) }\end{array}$ & $\begin{array}{l}\text { Residual } \\
\text { drawdown } \\
(\text { feet })^{1}\end{array}$ & $\begin{array}{c}\text { Time } \\
\text { (minutes) }\end{array}$ & $\begin{array}{l}\text { Water } \\
\text { leve } 1 \\
\text { (feet) }\end{array}$ & $\begin{array}{l}\text { Res Idua } 1 \\
\text { drawdown } \\
\text { (feet) } 1\end{array}$ \\
\hline 0.25 & 479.70 & 22.40 & 5.00 & 457.80 & 0.50 \\
\hline 0.50 & 456.85 & -0.45 & 6.00 & 457.75 & 0.45 \\
\hline 0.75 & 456.20 & -1.10 & 11.00 & 457.70 & 0.40 \\
\hline 1.00 & 456.20 & $-1 \cdot 10$ & 12.00 & 457.65 & 0.35 \\
\hline 1.25 & 456.20 & -1.10 & 13.00 & 457.65 & 0.35 \\
\hline 1.50 & 456.20 & -1.10 & 15.00 & 457.65 & 0.35 \\
\hline 1.75 & 456.20 & -1.10 & 18.00 & 457.65 & 0.35 \\
\hline 2.00 & 456.20 & -1.10 & 20.00 & 457.60 & 0.30 \\
\hline 2.25 & 456.20 & -1.10 & 23.00 & 457.60 & 0.30 \\
\hline 2.50 & 456.20 & -1.10 & 24.00 & 457.55 & 0.25 \\
\hline 2.75 & 456.20 & -1.10 & 25.00 & 457.55 & 0.25 \\
\hline 3.00 & 457.10 & -0.20 & 30.00 & 457.55 & 0.25 \\
\hline 3.25 & 457.95 & 0.65 & 35.00 & 457.50 & 0.20 \\
\hline 3.50 & 457.85 & 0.55 & 40.00 & 457.50 & 0.20 \\
\hline 3.75 & 457.80 & 0.50 & 45.00 & 457.45 & 0.15 \\
\hline 4.00 & 457.80 & 0.50 & 55.00 & 457.45 & 0.15 \\
\hline 4.25 & 457.80 & 0.50 & 68.00 & 457.45 & 0.15 \\
\hline
\end{tabular}

1 This well does not have a foot valve; consequently, when the pump is turned of $f$, an intial slug of water 18 delivered to the well as the water in the discharge pipe drains down through the pump (minus values). 


\section{$\underline{\mathrm{CE}-\mathrm{VF}-2}$}

We11 CE-VF-2 in Coyote Spring Valley, Lincoln County, is adjacent to U.S. Highway 93, approximately 4 miles north of the intersection of U.S. Highway 93 and State Route 168 (figure 1). The well site is east of Pahranagat Wash in Coyote Spring Valley.

Drilling began on December 15, 1980, and was completed sometime before April 1981 (exact date not known). The reported total depth is 1,221 feet and the water table was near 611 feet below land surface. Dolomitic limestone bedrock was penetrated at 850 feet. The well is cased with 10-inch-diameter blank casing from 0 to 860 feet and is uncased from 860 to 1,221 feet. The casing was set with cement from the surface to 50 feet and from 840 to 860 feet. Nongraded gravel pack was placed in the annulus from 50 to 840 feet. The drilling penetration rate and lithologic $\log$ for $\mathrm{CE}-\mathrm{VF}-2$ are shown in figure 24, and geophysical we11 logs are shown in figure 25. Another we11, CE-VF-1, was drilled as an observation well approximately 300 feet north of CE-VF-2. A reported depth of 714 feet was attained, bottoming in basin-fill deposits. The water level is 593 feet below land surface, indicating a vertical gradient between $\mathrm{CE}-\mathrm{VF}-2$ and $\mathrm{CE}-\mathrm{VF}-1$.

We11 CE-VF-2 was tested by the Geological Survey in February of 1986. The well was developed initially in 1981 by bailing 25 bails per day for $5 \frac{1}{2}$ days ( 16.5 gallons per bail). The pump, flow-meter assemb1y, and pressure transducer used in 1986 were the same as those used in the test at CSV-2. The pump intake was set at 707 feet and the discharge was piped 80 feet away from the site. The well was pumped at $77 \mathrm{ga} 1 / \mathrm{min}$ for 14 hours, and recovery took 2 hours. The pump stopped unexpectedly due to mechanical failure and, as a result, the exact time for the beginning of the recovery portion of the test is uncertain within 1 to 4 minutes. The initial recovery measurements exhibit a great deal of scatter due to rapid oscillations of the water in the well or to problems with the pressure transducer or recorder. Despite these initial difficulties, the long-term recovery of water levels was accurately measured. See tables 12 and 13 and figures 26 and 27. Measurements of water levels in an observation well 300 feet away, $C E-V F-1$, showed no change during the 14-hour test. The observation well does not penetrate the carbonate aquifer. 


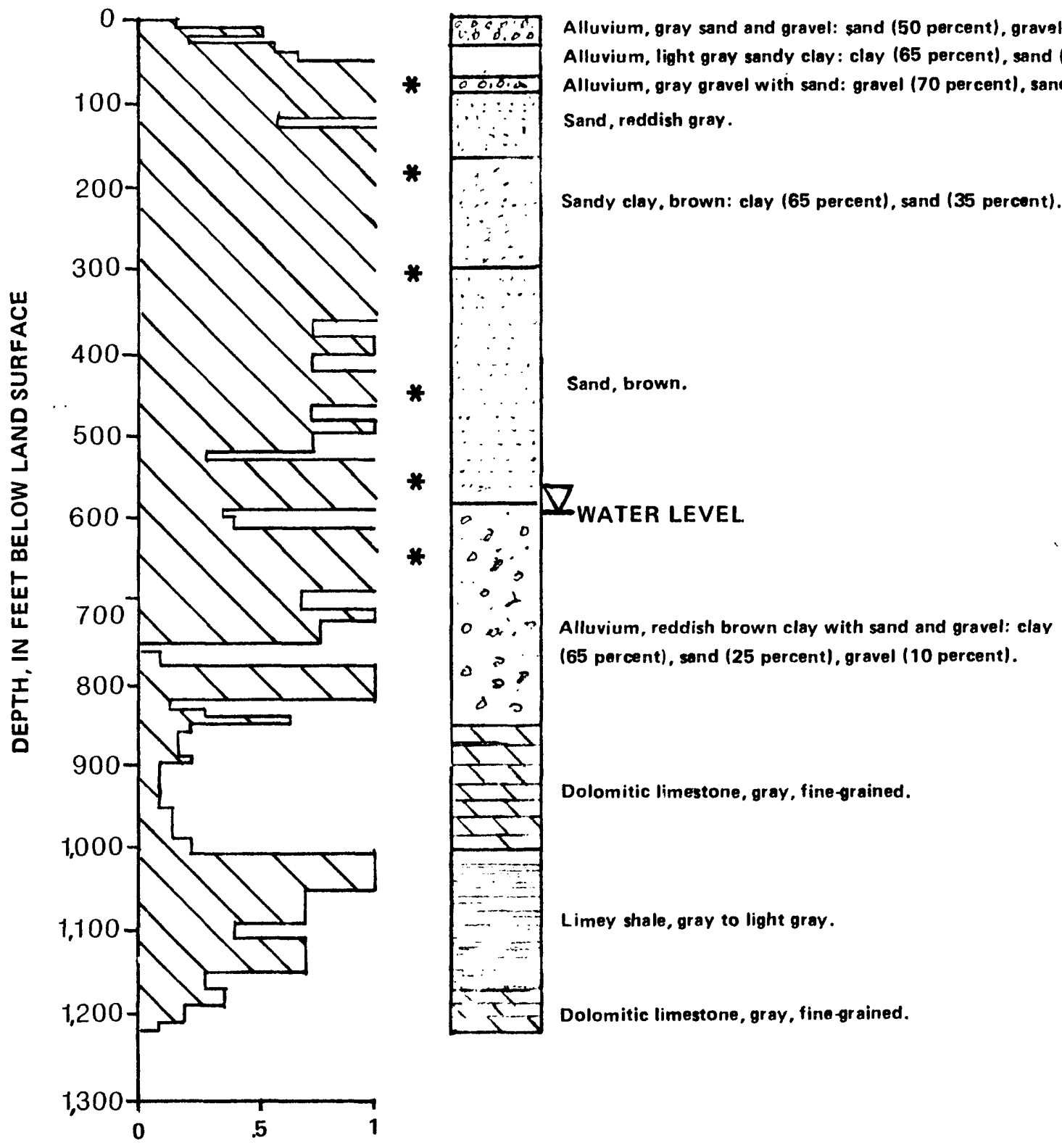

PENETRATION RATE, IN FEET PER MINUTE

FIGURE 24.--Drilling penetration rate and lithology for MX test well CE-VF-2.

Asterisk indicates penetration rate exceeding 1.0 foot per minute. 

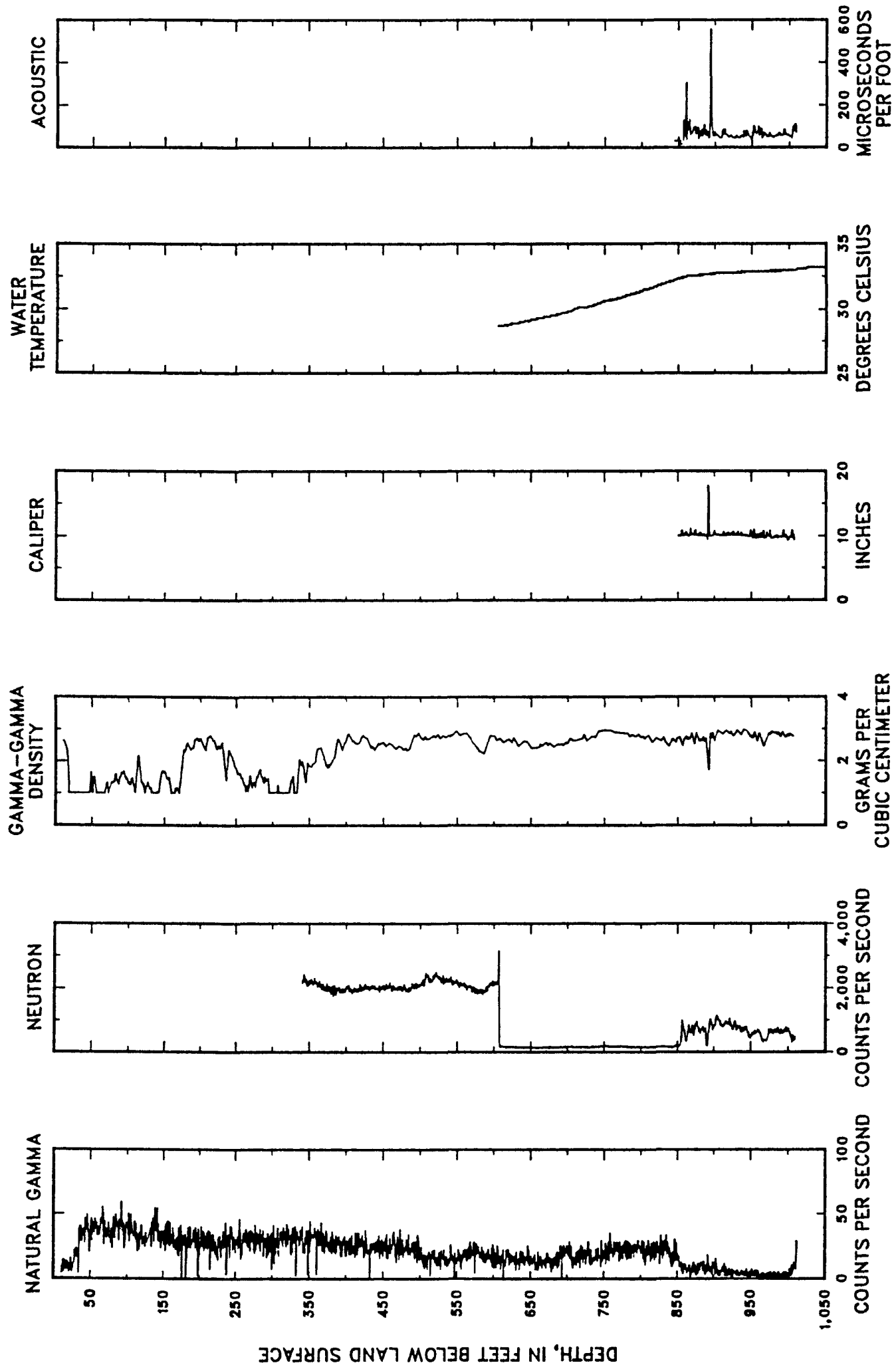

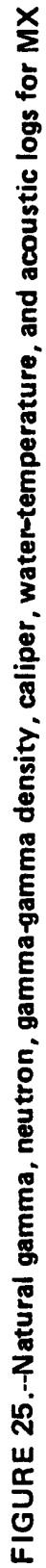




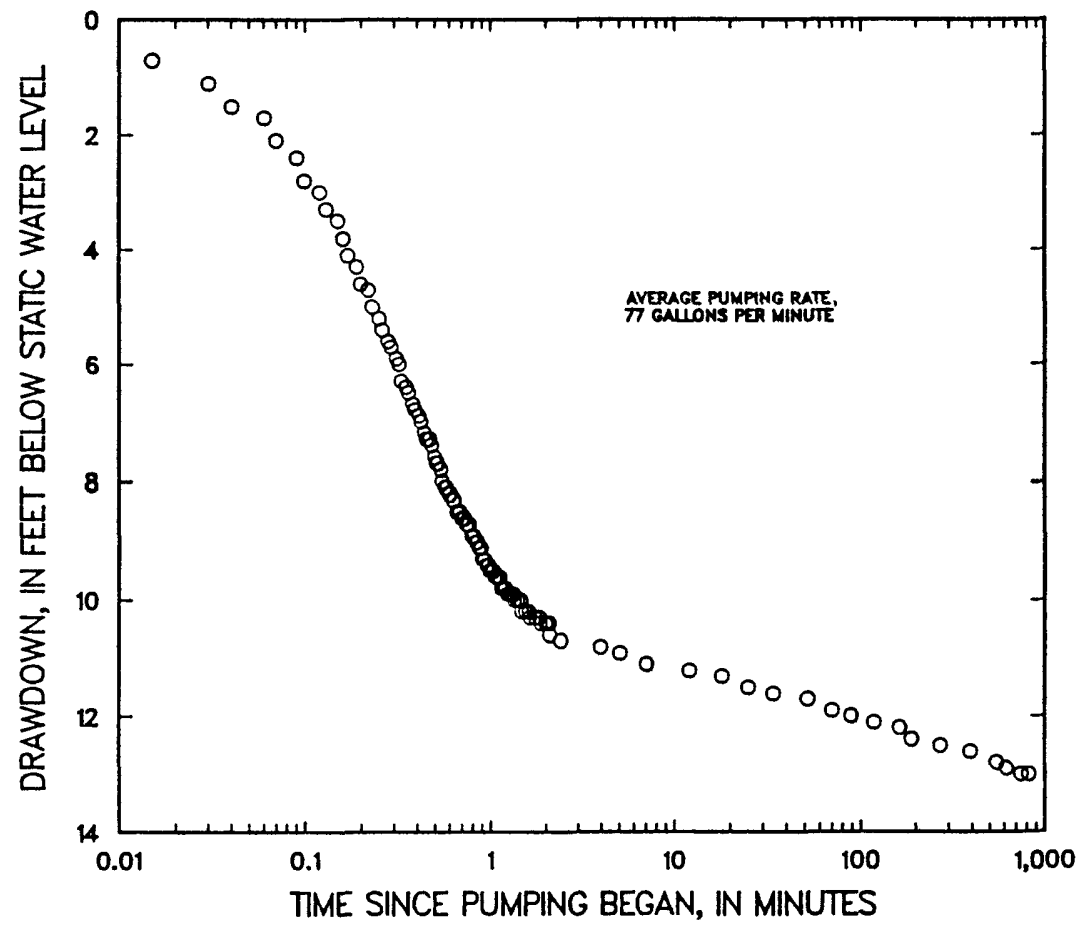

FIGURE 26.--Drawdown versus time at MX test well CE-VF-2, February 6, 1986.

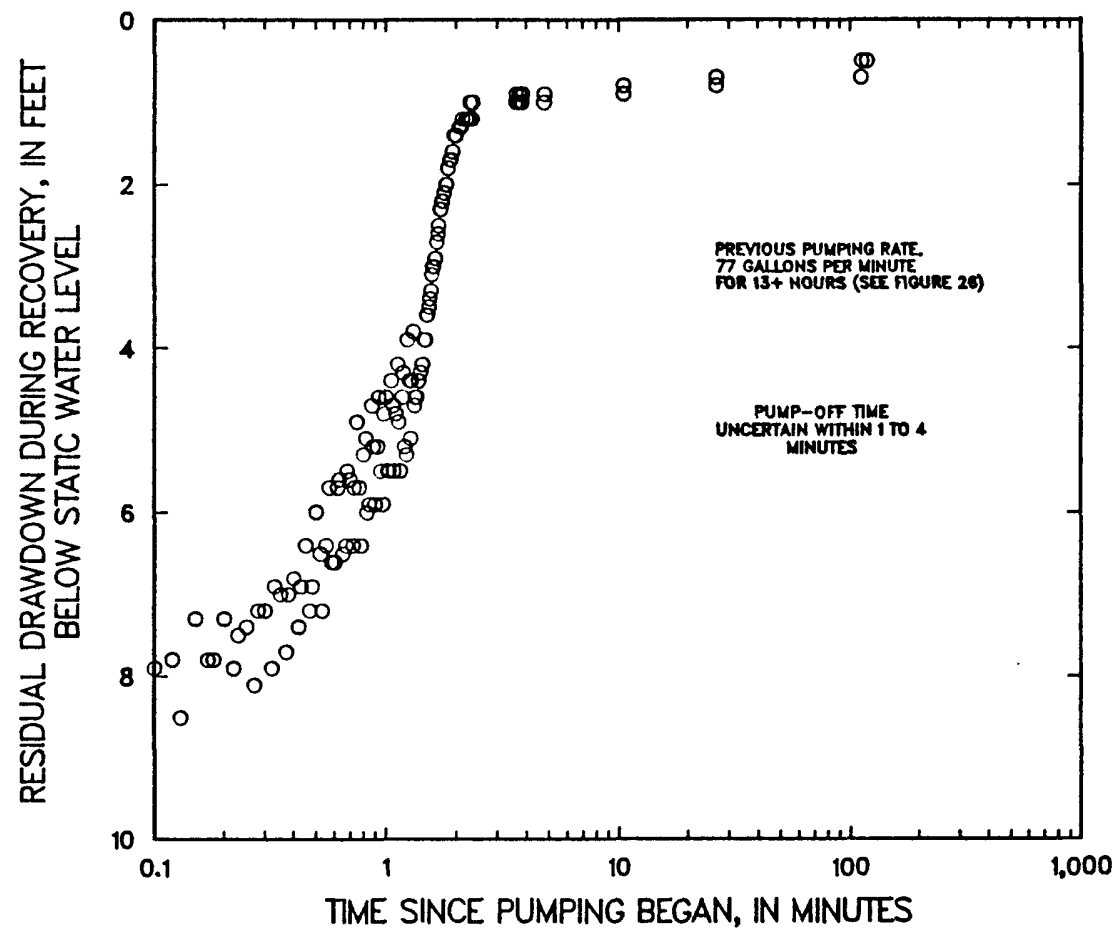

FIGURE 27.--Residual drawdown versus time at MX test well CE-VF-2, February 6, 1986. 
TABLE 12.--Water levels during a 14-hour, constant-discharge aquifer test at MX test well C' $-V F-2$, F'ebruary 6, 1986. Static water level is 604.3 feet

ITime, mlnutes since pumplng began; water level, feet below land surface; drawdown, calculated drawdown of water levels in feet below stat 1c water level; pumping rate, spot-checked during test (average pumping rate during entire test determined from flow totallzer was 76.8 gallons per minute)]

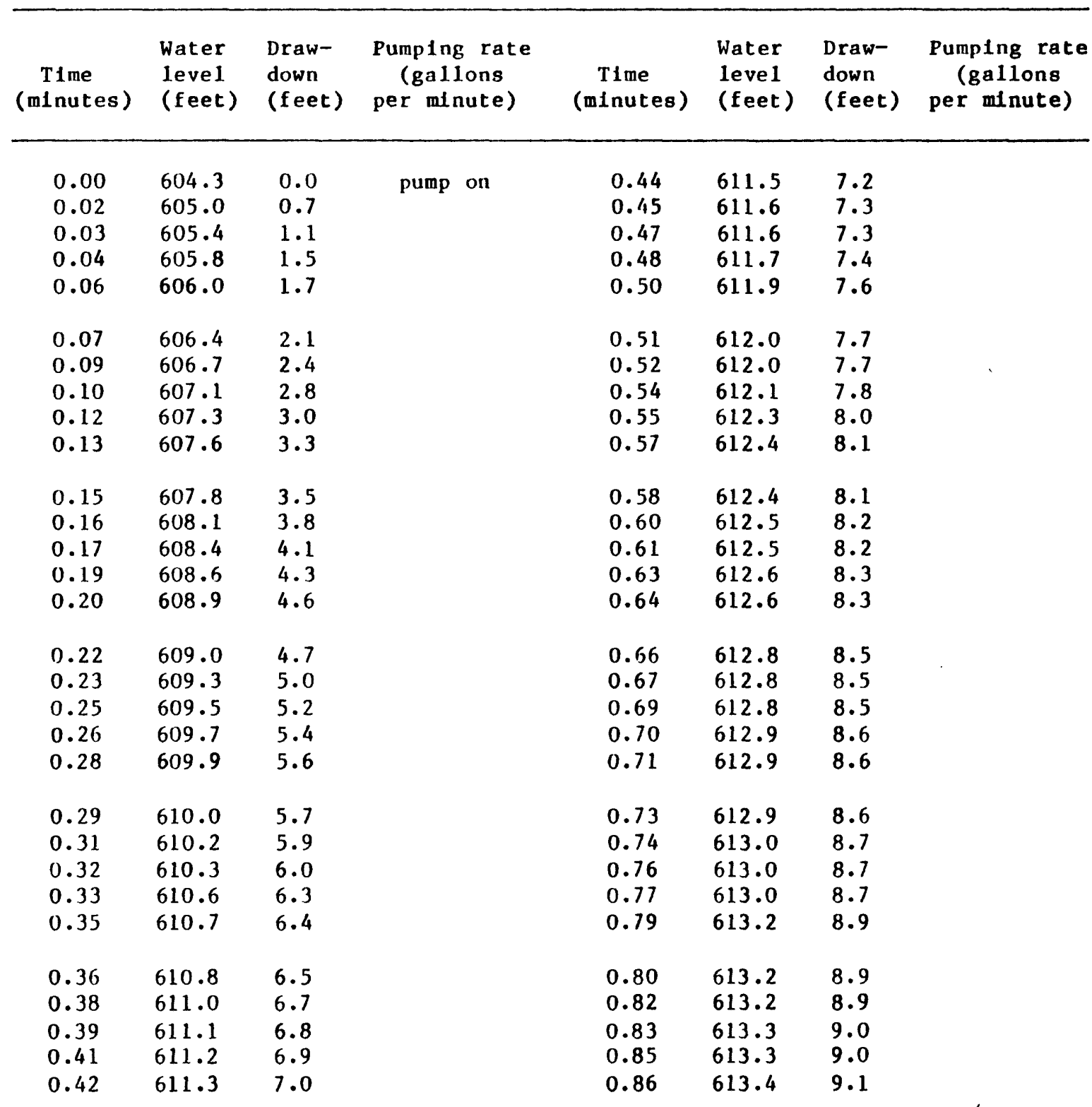


TABLE 12.--Water levels during a 14-hour, constant-discharge ayuifer test at $M X$ test well C'E-VE-2, F'ebruary 6, 1986--Cont Inued

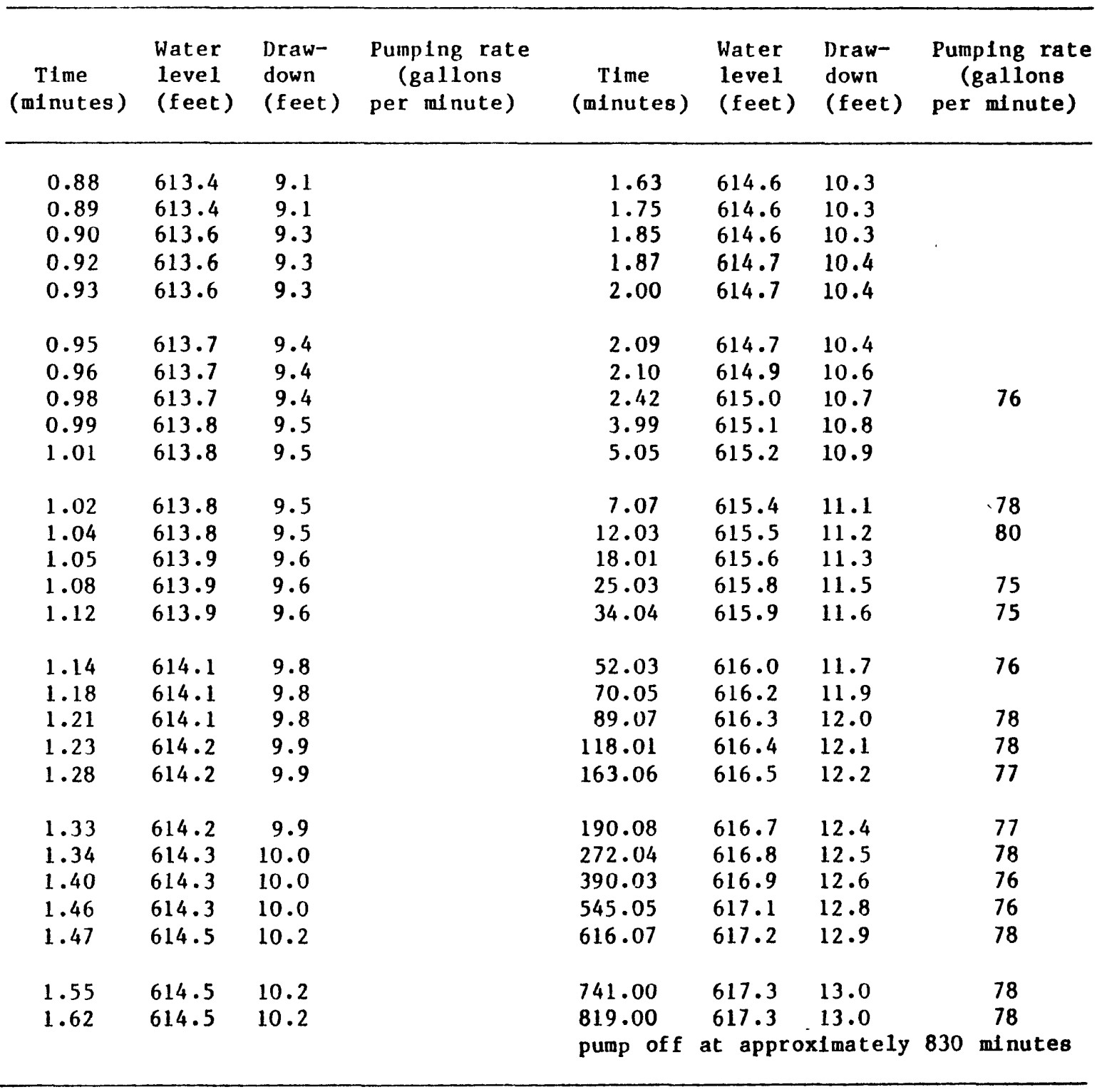


TABLE 13.--Water levels during recovery from a 14-hour, constant-discharge aquifer test at $M X$ test well $C^{\prime} b^{\prime}-V E-Z$, February $6-7,1986^{\circ}$

[TIme, arbitrary time since pumping ended (arbitrary because pump-off time is uncertain within 1 to 4 minutes); water level, feet below land surface; residual drawdown, calculated drawdown of water levels remalning below static water level]

\begin{tabular}{|c|c|c|c|c|c|}
\hline $\begin{array}{c}\text { Time } \\
\text { (minutes) }\end{array}$ & $\begin{array}{l}\text { Water } \\
\text { leve } 1 \\
\text { (feet) }\end{array}$ & $\begin{array}{c}\text { Res Idual } \\
\text { drawdown } \\
\text { (f eet) }\end{array}$ & $\begin{array}{c}\text { Time } \\
\text { (minutes) }\end{array}$ & $\begin{array}{l}\text { Water } \\
\text { level } \\
\text { (feet) }\end{array}$ & $\begin{array}{c}\text { Residual } \\
\text { drawdown } \\
\text { (feet) }\end{array}$ \\
\hline $\begin{array}{l}0.025 \\
0.03\end{array}$ & $\begin{array}{l}612 \cdot 5 \\
612.4\end{array}$ & $\begin{array}{l}8.2 \\
8.1\end{array}$ & $\begin{array}{l}0.52 \\
0.53\end{array}$ & $\begin{array}{l}610.8 \\
611.5\end{array}$ & $\begin{array}{l}6.5 \\
7.2\end{array}$ \\
\hline 0.05 & 612.9 & 8.6 & 0.55 & 610.7 & 6.4 \\
\hline 0.07 & 612.0 & 7.7 & 0.57 & 610.0 & 5.7 \\
\hline 0.08 & 612.5 & 8.2 & 0.58 & 610.9 & 6.6 \\
\hline 0.10 & 612.2 & 7.9 & 0.60 & 610.9 & 6.6 \\
\hline 0.12 & 612.1 & 7.8 & 0.62 & 610.0 & 5.7 \\
\hline 0.13 & 612.8 & 8.5 & 0.63 & 609.9 & 5.6 \\
\hline 0.15 & 611.6 & 7.3 & 0.65 & 610.8 & 6.5 \\
\hline 0.17 & 612.1 & 7.8 & 0.67 & 610.7 & 6.4 \\
\hline 0.18 & 612.1 & 7.8 & 0.68 & 609.8 & 5.5 \\
\hline 0.20 & 611.6 & 7.3 & 0.70 & 609.9 & 5.6 \\
\hline 0.22 & 612.2 & 7.9 & 0.72 & 610.7 & 6.4 \\
\hline 0.23 & 611.8 & 7.5 & 0.73 & 610.0 & 5.7 \\
\hline 0.25 & 611.7 & 7.4 & 0.75 & 609.2 & 4.9 \\
\hline 0.27 & 612.4 & 8.1 & 0.77 & 610.0 & 5.7 \\
\hline 0.28 & 611.5 & 7.2 & 0.78 & 610.7 & 6.4 \\
\hline 0.30 & 611.5 & 7.2 & 0.80 & 609.6 & 5.3 \\
\hline 0.32 & 612.2 & 7.9 & 0.82 & 609.4 & 5.1 \\
\hline 0.33 & 611.2 & 6.9 & 0.83 & 610.3 & 6.0 \\
\hline 0.35 & 611.3 & 7.0 & 0.85 & 610.2 & 5.9 \\
\hline 0.37 & 612.0 & 7.7 & 0.87 & 609.0 & 4.7 \\
\hline 0.38 & 611.3 & 7.0 & 0.88 & 609.5 & 5.2 \\
\hline 0.40 & 611.1 & 6.8 & 0.90 & 610.2 & 5.9 \\
\hline 0.42 & 611.7 & 7.4 & 0.92 & 609.5 & 5.2 \\
\hline 0.43 & 611.2 & 6.9 & 0.93 & 608.9 & 4.6 \\
\hline 0.45 & 610.7 & 6.4 & 0.95 & 609.8 & 5.5 \\
\hline 0.47 & 611.5 & 7.2 & 0.97 & 610.2 & 5.9 \\
\hline 0.48 & 611.2 & 6.9 & 0.98 & 609.1 & 4.8 \\
\hline 0.50 & 610.3 & 6.0 & 1.00 & 608.9 & 4.6 \\
\hline
\end{tabular}


TABLE 13.--Water levels during recovery from a 14-hour, constant-discharge aquifer test at $M X$ test well $C E-V E-Z$, E'ebruary 6-7, 1986--Cont 1nued

\begin{tabular}{|c|c|c|c|c|c|}
\hline $\begin{array}{c}\text { Time } \\
\text { (minutes) }\end{array}$ & $\begin{array}{l}\text { Water } \\
\text { leve } 1 \\
\text { (feet) }\end{array}$ & $\begin{array}{c}\text { Res Idual } \\
\text { drawdown } \\
\text { (feet) }\end{array}$ & $\begin{array}{c}\text { Time } \\
\text { (minutes) }\end{array}$ & $\begin{array}{l}\text { Water } \\
\text { level } \\
\text { (feet) }\end{array}$ & $\begin{array}{c}\text { Residual } \\
\text { drawdown } \\
\text { (feet) }\end{array}$ \\
\hline 1.02 & 609.8 & 5.5 & 1.60 & 607.3 & 3.0 \\
\hline 1.03 & 609.8 & 5.5 & 1.62 & 607.2 & 2.9 \\
\hline 1.05 & 608.7 & 4.4 & 1.63 & 607.2 & 2.9 \\
\hline 1.07 & 609.0 & 4.7 & 1.65 & 607.0 & 2.7 \\
\hline 1.08 & 609.8 & 5.5 & 1.67 & 606.9 & 2.6 \\
\hline 1.10 & 609.1 & 4.8 & 1.68 & 606.8 & 2.5 \\
\hline 1.12 & 608.5 & 4.2 & 1.70 & 606.6 & 2.3 \\
\hline 1.13 & 609.2 & 4.9 & 1.72 & 606.6 & 2.3 \\
\hline 1.15 & 609.8 & 5.5 & 1.73 & 606.5 & 2.2 \\
\hline 1.17 & 608.9 & 4.6 & 1.75 & 606.5 & 2.2 \\
\hline 1.18 & 608.6 & 4.3 & 1.77 & 606.4 & 2.1 \\
\hline 1.20 & 609.5 & 5.2 & 1.78 & 606.4 & 2.1 \\
\hline 1.22 & 609.6 & 5.3 & 1.80 & 606.3 & 2.0 \\
\hline 1.23 & 608.2 & 3.9 & 1.82 & 606.3 & 2.0 \\
\hline 1.25 & 608.7 & 4.4 & 1.83 & 606.1 & 1.8 \\
\hline 1.27 & 609.4 & 5.1 & 1.85 & 606.1 & 1.8 \\
\hline 1.28 & 608.7 & 4.4 & 1.87 & 606.0 & 1.7 \\
\hline 1.30 & 608.1 & 3.8 & 1.88 & 606.0 & 1.7 \\
\hline 1.32 & 609.0 & 4.7 & 1.90 & 606.0 & 1.7 \\
\hline 1.33 & 608.9 & 4.6 & 1.92 & 605.9 & 1.6 \\
\hline 1.35 & 608.9 & 4.6 & 1.93 & 605.9 & 1.6 \\
\hline 1.37 & 608.7 & 4.4 & 1.95 & 605.7 & 1.4 \\
\hline 1.38 & 608.7 & 4.4 & 2.00 & 605.7 & 1.4 \\
\hline 1.40 & 608.6 & 4.3 & 2.05 & 605.6 & 1.3 \\
\hline 1.42 & 608.5 & 4.2 & 2.10 & 605.6 & 1.3 \\
\hline 1.43 & 608.5 & 4.2 & 2.12 & 605.5 & 1.2 \\
\hline 1.45 & 608.2 & 3.9 & 2.20 & 605.5 & 1.2 \\
\hline 1.47 & 608.2 & 3.9 & 2.25 & 605.5 & 1.2 \\
\hline 1.48 & 607.9 & 3.6 & 2.28 & 605.3 & 1.0 \\
\hline 1.50 & 607.9 & 3.6 & 2.30 & 605.5 & 1.2 \\
\hline 1.52 & 607.8 & 3.5 & 2.33 & 605.5 & 1.2 \\
\hline 1.53 & 607.7 & 3.4 & 2.35 & 605.3 & 1.0 \\
\hline 1.55 & 607.6 & 3.3 & 3.58 & 605.3 & 1.0 \\
\hline 1.57 & 607.4 & 3.1 & 3.60 & 605.2 & 0.9 \\
\hline 1.58 & 607.3 & 3.0 & 3.62 & 605.3 & 1.0 \\
\hline
\end{tabular}


TABLE 13.--Water levels during recovery from a 14-hour, constant-discharge aquifer test at MX test well $C b^{\prime}-V F-2$, Eebruary 6-7, 1986--Cont Inued

\begin{tabular}{llllll}
\hline $\begin{array}{c}\text { Time } \\
\text { (minutes) }\end{array}$ & $\begin{array}{c}\text { Water } \\
\text { leve1 } \\
\text { (feet) }\end{array}$ & $\begin{array}{c}\text { Residual } \\
\text { drawdown } \\
\text { (feet) }\end{array}$ & $\begin{array}{c}\text { Time } \\
\text { (minutes) }\end{array}$ & $\begin{array}{c}\text { Water } \\
\text { leve) } \\
\text { (feet) }\end{array}$ & $\begin{array}{c}\text { Residual } \\
\text { drawdown } \\
\text { (feet) }\end{array}$ \\
\hline & & & & & \\
3.72 & 605.3 & 1.0 & 4.78 & 605.2 & 0.9 \\
3.73 & 605.2 & 0.9 & 10.42 & 605.2 & 0.9 \\
3.75 & 605.3 & 1.0 & 10.43 & 605.1 & 0.8 \\
3.77 & 605.3 & 1.0 & 26.32 & 605.1 & 0.8 \\
3.78 & 605.2 & 0.9 & 26.33 & 605.0 & 0.7 \\
& & & 110.33 & 605.0 & 0.7 \\
3.80 & 605.3 & 1.0 & 11.33 & 604.8 & 0.5 \\
3.82 & 605.3 & 1.0 & 117.33 & 604.8 & 0.5 \\
3.85 & 605.2 & 0.9 & & & \\
\hline 4.77 & 605.3 & 1.0 & & & \\
\hline
\end{tabular}




\section{SHV-1}

We11 SHV-1 in Hidden Valley, Clark County, is adjacent to U.S. Highway 93, approximately 16.5 miles south of the intersection of U.S. Highway 93 and State Route 168 (figure 1).

This well was drilled sometime before 1958 on U.S. Bureau of Land Management land, apparently for use as a stock we11, and is now abandoned. The well was plugged above the water table until the Geological Survey redrilled it to a depth of 920 feet in December 1985. The water table is 832.3 feet below the land surface. The caliper log indicates 5-inchdiameter casing to a depth of 45 feet and 6-inch uncased hole below. The drilling history and well construction are unknown. Geophysical we11 logs of this well are shown in figure 28 . 

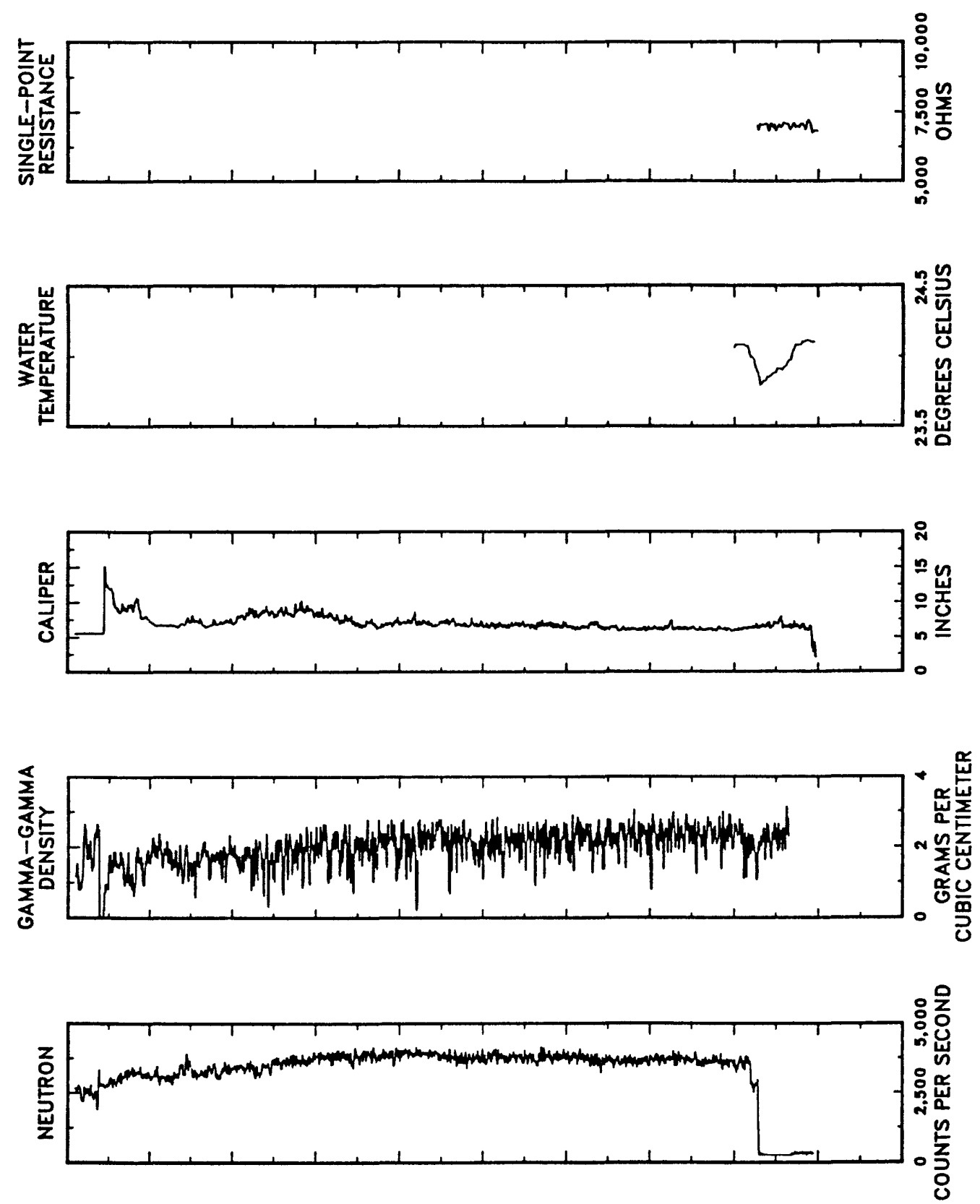

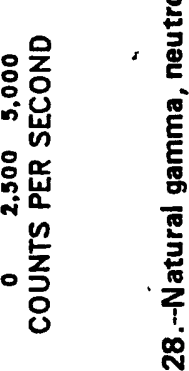

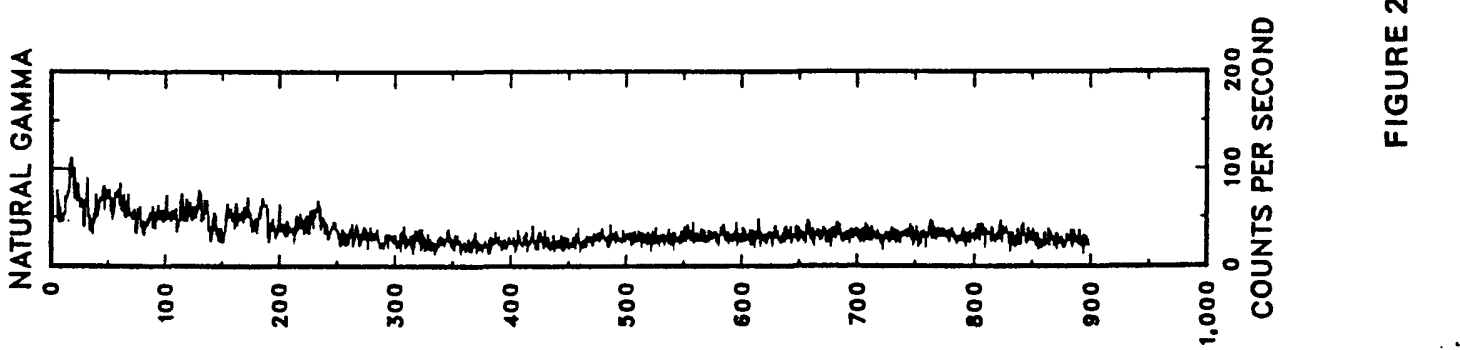

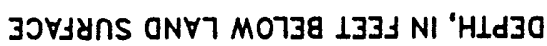


REFERENCES CITED

Ertec Western, Inc., 1981, Water resources program, results of regional carbonate aquifer testing, Coyote Spring Valley, Nevada: Long Beach, Calif., Department of the Air Force, MX Siting Investigation Water Resources Report E-TR-57, 190 p.

Rush, F. E., 1968, Index of hydrographic areas in Nevada: Nevada Division of Water Resources Information Report 6, $38 \mathrm{p}$. 